\title{
Mapping between RDF and XML with XSPARQL
}

\author{
Stefan Bischof - Stefan Decker • \\ Thomas Krennwallner • Nuno Lopes • \\ Axel Polleres
}

Received: 2 December 2011 / Revised: 4 May 2012 / Accepted: 11 June 2012 / Published online: 4 September 2012

(C) Springer-Verlag 2012

\begin{abstract}
One promise of Semantic Web applications is to seamlessly deal with heterogeneous data. The Extensible Markup Language (XML) has become widely adopted as an almost ubiquitous interchange format for data, along with transformation languages like XSLT and XQuery to translate data from one XML format into another. However, the more recent Resource Description Framework (RDF) has become another popular standard for data representation and exchange, supported by its own query language SPARQL, that enables extraction and transformation of RDF data. Being able to work with XML and RDF using a common framework eliminates several unnecessary steps that are currently required when handling both formats side by side. In this paper we present the XSPARQL language that, by combining XQuery and SPARQL, allows to query XML and $\mathrm{RDF}$ data using the same framework and transform data from
\end{abstract}

S. Bischof · A. Polleres

Siemens AG Österreich, Siemensstrasse 90, 1210 Vienna, Austria

e-mail: bischof.stefan@siemens.com

A. Polleres

e-mail: axel.polleres@siemens.com

S. Decker · N. Lopes $(\bowtie)$

Digital Enterprise Research Institute (DERI), National University of Ireland, Galway, Ireland

e-mail: nuno.lopes@deri.org

S. Decker $\cdot$ N. Lopes

IDA Business Park, Lower Dangan, Galway, Ireland

e-mail: stefan.decker@deri.org

T. Krennwallner

Institute of Information Systems, Vienna University of Technology,

Favoritenstraße 9-11, 1040 Vienna, Austria

e-mail: tkren@kr.tuwien.ac.at one format into the other. We focus on the semantics of this combined language and present an implementation, including discussion of query optimisations along with benchmark evaluation.

Keywords Query processing $\cdot \mathrm{XML} \cdot \mathrm{RDF} \cdot \mathrm{SPARQL}$. XQuery $\cdot$ XSPARQL

\section{Introduction}

XML [18] has become a well established and widely adopted interchange format for data on the Web. Accompanying standards, such as XSL Transformations (XSLT) by Kay [42] and, more recently, XQuery by Chamberlin et al. [22], both based on the XML Path Language (XPath) [11], are often used to query XML data and convert between different XML representations.

In the effort to convert the Web into a Semantic Web, the Resource Description Framework (RDF) [46,39] has become the language of choice for modelling, interlinking, and merging data. RDF data and applications that consume this data are becoming increasingly present on the Web. Opposed to the tree structure of XML, RDF structures data in sets of triples, representing edges of a directed, labelled graph. Querying RDF graphs and converting between them can be performed using SPARQL [54], the W3C recommended query language for RDF.

In many applications combining and converting between $\mathrm{XML}$ and RDF data is a useful but often not trivial task. The importance of this issue is acknowledged within the W3C, for instance in the working groups on Gleaning Resource Descriptions from Dialects of Languages (GRDDL) by Connolly [24] and Semantic Annotations for WSDL (SAWSDL) by Farrell and Lausen [29]. As we will show, common 
approaches for transformations between XML and RDF, which rely on the standard XML serialisation of RDF by Beckett and McBride [9] and on XML technologies, e.g., XSLT, have several disadvantages. While both XQuery and SPARQL languages operate on different data models, respectively, the XQuery and XPath Data Model (XDM) [30] for $\mathrm{XML}$ and RDF, we show that the merge of both query languages in the novel language XSPARQL has the potential to finally bring XML and RDF closer together. XSPARQL provides concise and intuitive solutions for mapping between XML and RDF in either direction: operations where both XQuery and SPARQL struggle. In fact it is not possible to use SPARQL alone for such transformations since the SPARQL query language does not provide the possibility of handling XML data. On the other side, the only way to work with RDF data within XQuery is by relying on the RDF/XML serialisation for RDF graphs. As we show in Sect. 2, this approach is hard to implement due to the different possible serialisations in RDF/XML for a single RDF graph. An additional use for XSPARQL is the conversion between RDF graphs. XSPARQL extends SPARQL's expressiveness for such transformations, by allowing, for instance, nested XSPARQL queries in the graph construction step.

Since its first version by Akhtar et al. [4], XSPARQL has gained community interest and practical use cases have been presented in a W3C Member Submission [49]. Based on these experiences, the present article makes the following main contributions:

- we present syntax and formal semantics of XSPARQL based on the XQuery Formal Semantics by Draper et al. [27]. In comparison with our initial publication, we improved the treatment of nested queries over RDF with respect to blank nodes and allow for assignment of RDF graphs to variables;

- our implementation of XSPARQL is based on rewriting an XSPARQL query into a semantically equivalent XQuery query; as opposed to the preliminary version of this rewriting by Akhtar et al. [4], in this paper we present a more tightly integrated, new prototype implementing several new features;

- we prove various properties of XSPARQL and show soundness and completeness of the new tighter query rewriting;

- we present a set of optimisations for complex queries (containing nested XSPARQL queries) and show their correctness;

- we introduce a novel benchmark suite (XMarkRDF) that extends the XMark XML Benchmark suite by also considering RDF as a data format; and

- based on the XMarkRDF suite, we present benchmark evaluation of the new XSPARQL prototype and compare it with a related system. Furthermore, we discuss the performance impact of the proposed optimisations.

The article is organised as follows: Sect. 2 will illustrate our main motivation to come up with a new language by discussing drawbacks of existing technologies for transformations between RDF and XML. In Sect. 3 we will briefly review the main characteristics of the XQuery and SPARQL query languages and, in Sect. 4, present their combination in the form of the XSPARQL language by defining the formal semantics and showing semantic properties of the novel language. Section 5 shows the architecture and query rewriting techniques for a prototype implementation. Section 6 discusses query optimisation techniques that speed up the evaluation of XSPARQL queries. We compare XSPARQL with another prototype that combines SPARQL and XQuery in Sect. 7 and report on experimental results using the benchmark suite XMarkRDF. We also compare query response times of the presented optimisations, showing promising results. We conclude this work with a discussion of related works in Sect. 8 and wrap up in Sect. 9.

\section{Motivation: Lifting and Lowering}

XML can be viewed as a tree-like data representation format, with intermediate nodes of this tree being XML elements or attribute names, and the leaf nodes being either empty elements or textual attribute values and element content. The order of child nodes is relevant in XML. As opposed to this, RDF data, i.e., an RDF graph, is an unordered set of subjectpredicate-object triples, as follows:

Definition 1 (RDF Triple, RDF Graph) Given pairwise disjoint sets of URI references $\mathbb{U}$, blank nodes $\mathbb{B}$, and literals $\mathbb{L},{ }^{1}$ a triple $(s, p, o) \in \mathbb{U B} \times \mathbb{U} \times \mathbb{U B} \mathbb{L}$ (often written as a "statement" 's $p o$.') is called an RDF triple; sets of RDF triples are called $R D F$ graphs. We call elements of $U \mathbb{B L} R D F$ terms.

Besides the normative syntax to exchange RDF using XML, RDF/XML [9], there are various serialisation formats for RDF, such as RDFa [2], a format that allows one to embed RDF within (X)HTML, or non-XML representations such as the more human-readable Turtle [7] syntax. Since data in RDF may be considered on a higher level of abstraction than semi-structured XML data, the translation from XML to RDF is often called lifting, while the opposite direction is called lowering. The importance of converting data between the XML and RDF formats has been acknowledged

\footnotetext{
${ }^{1}$ For brevity we will denote the concatenation of sets by concatenating their names, e.g., $\mathbb{U} \cup \mathbb{B}$ is represented as $\mathbb{U B}$.
} 


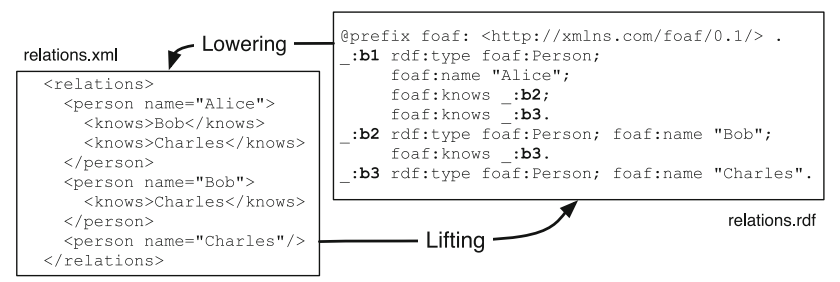

Fig. 1 From XML to RDF and back: "lifting" and "lowering"

within the $\mathrm{W} 3 \mathrm{C}$ in several related standardisation efforts, such as GRDDL and SAWSDL. The GRDDL working group addressed the issue of extracting RDF data out of existing (X)HTML Web pages (lifting). Likewise, in the Semantic Web Services community, the SAWSDL working group aimed at defining mechanisms (and link them in Web service descriptions) to generate XML messages sent to Web services from RDF data (lowering) and vice versa extract RDF from service result messages in XML (lifting) [see 29,44]. Both GRDDL and SAWSDL use XSLT (although they acknowledge that other mechanisms could be used) in their examples to perform lifting and lowering. In the following, let us illustrate some drawbacks of this approach.

As a running example throughout this paper we use a mapping between a custom XML format and RDF as shown in Fig. 1 (using Turtle syntax for illustration). The task is, in both directions, to extract for all persons the names of people they know. URIs denoting predicates and terms in a particular domain are typically collected under a common namespace in RDF with a designated prefix, such as RDF core terms in the namespace http://www.w3.org/1999/02/22-rdfsyntax-ns\# using prefix rdf: or terms of the FOAF [20] ontology in the namespace http://xmlns.com/foaf/0.1/ using prefix foaf : . ${ }^{2}$

Blank nodes are represented in Turtle by the prefix '_:' followed by an identifier/label, or by square brackets '[]'. Blank nodes play a special role in RDF's data model: they allow to model unknown nodes or incomplete data, akin to existential variables. Regarding the serialisation in Turtle that means, if we would replace _: b1 in Fig. 1 by _: $x$, it would represent an equivalent RDF graph.

$\mathrm{RDF} / \mathrm{XML}$ [9] is the recommended syntax for RDF, using $\mathrm{XML}$ as the underlying representation model. This format enables the use of XML tools such as XSLT or XQuery to translate between RDF/XML and other XML formats. However, such a transformation is greatly complicated by the flexibility the RDF/XML format offers in serialising RDF graphs. Therefore, tools that handle RDF/XML as XML data (and not as a sets of triples) need to take different possible representations into account. Figure 2 shows four versions of a subset of the RDF data from our running example that represent

\footnotetext{
${ }^{2}$ In listings and figures we sometimes abbreviate well-known namespace URIs with "...".
}

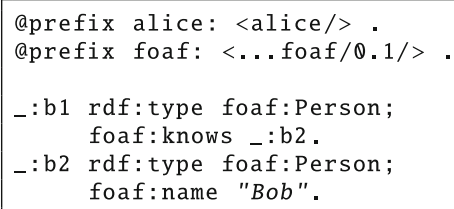

(a) Turtle

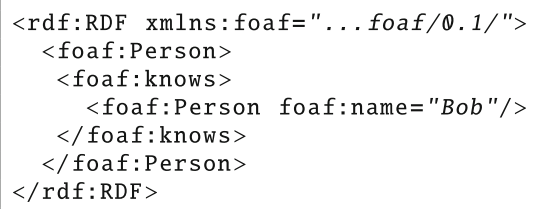

(b) Concise XML/RDF

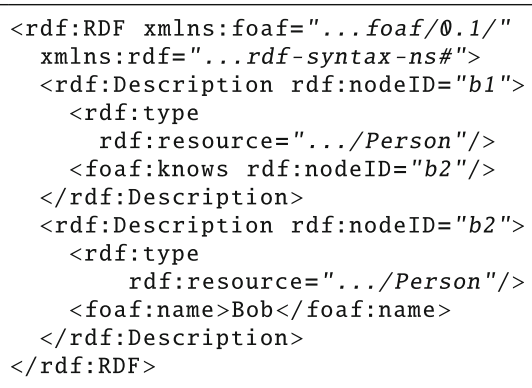

(c) $\mathrm{XML} / \mathrm{RDF}$

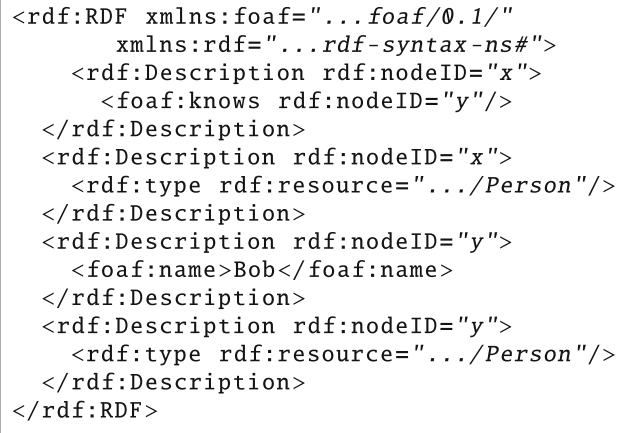

(d) Verbose XML/RDF

Fig. 2 Different representations of the same RDF graph

the same FOAF data. Figure 2a uses Turtle [7], a simple and readable textual format for RDF, inaccessible to pure XML processing tools though; the remaining three versions are all $\mathrm{RDF} / \mathrm{XML}$, ranging from concise (2b) to verbose (2d). These three RDF/XML variants represent different XML trees but the same RDF graph. Note that blank node identifiers may disappear or change through XML serialisation.

For our running example, let us attempt lifting and lowering transformations using XSLT (we will get to XQuery as another alternative in more detail later on). 


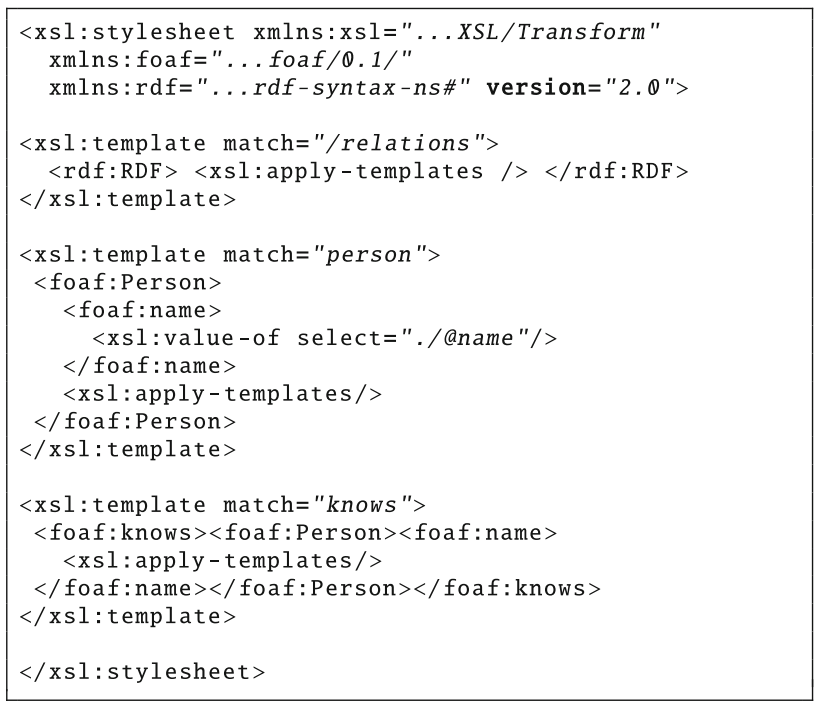

(a) XSL transform lifting.Xsl

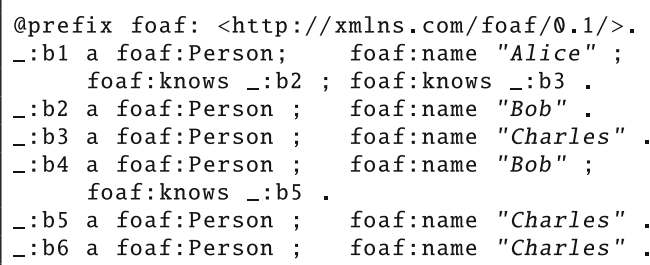

(b) Result in Turtle

Fig. 3 Lifting attempt by XSLT

Lifting. The XSLT stylesheet in Fig. 3a for instance, could be used to generate RDF/XML (in the format presented in Fig. 2b) from the relations.xml file in Fig. 1. However, this first attempt does not yet accomplish the intended transformation since unique identifiers are not created for each person. This is easy to see in the Turtle version of the result of this transformation (presented in Fig. 3b): while in our example names should uniquely identify a person, in this transformation the same person is potentially given several different blank nodes.

Although a proper lifting transformation catering for all possible XML serialisations is doable in XSLT, the corresponding stylesheet would need to be far more involved.

Lowering. The simple XSLT stylesheet lowering.xsl in Fig. 4b is an attempt to perform the lowering task directly from RDF/XML. However, this XSLT will break if the input $\mathrm{RDF} / \mathrm{XML}$ serialisation is in any other variant than the version in Fig. 2b. We could create a specific stylesheet for each of the presented variants, but creating one that handles all the possible RDF/XML forms would be much more complicated.

Apart from its syntactic ambiguities, processing RDF/ XML via XSLT also loses another feature of RDF, namely

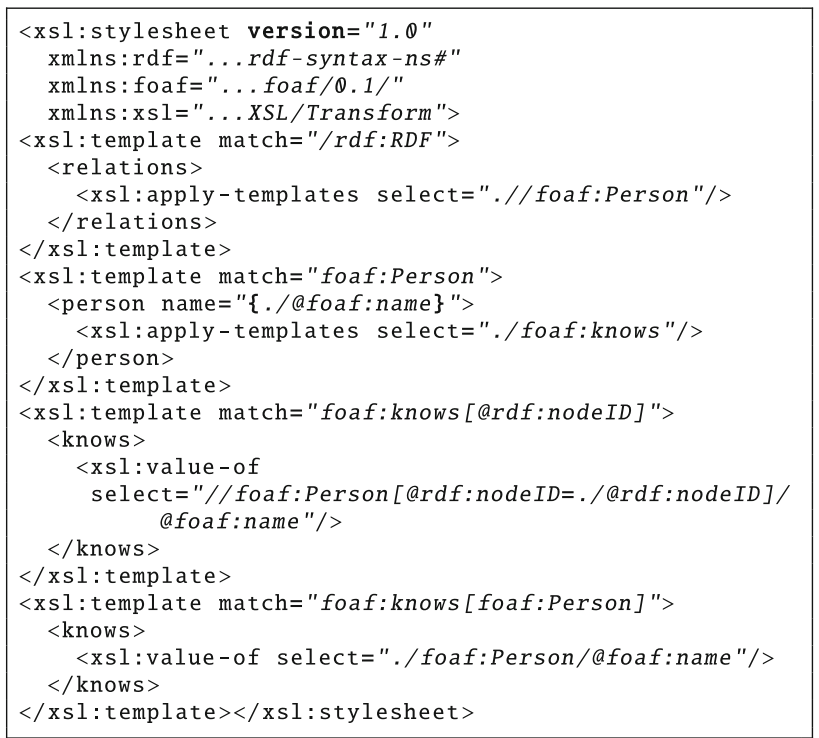

Fig. 4 Lowering using XSLT (lowering.xsl)

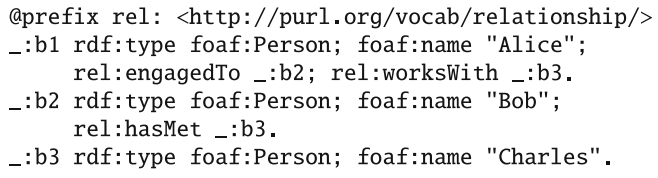

Fig. 5 RDF data using the relationship ontology

its interplay with ontological information, e.g., RDF Schema. RDF Schema [19] (RDFS) allows to express subclass or subproperty hierarchies, which can be exploited by RDF tools capable of ontological inference. The RDF data from Fig. 1 could-rather than foaf:knows-use predicates from the relationship ontology, ${ }^{3}$ which are all stated as subproperties of foaf: knows, as presented in Fig. 5. Similar considerations would apply if we attempted to perform the lifting and lowering using XQuery: since XML tools do not support ontological inference, we literally would need to implement an RDFS inference engine within XSLT or XQuery, to be able to implement a lowering mechanism that also works for this kind of RDF data. Given the availability of RDF tools and engines that readily offer RDFS support, this seems to be a dispensable exercise.

Benefits of an integrated language. In recognition of the above problems, the SAWSDL specification contains a nonnormative example which performs a lowering transformation as a sequence of a SPARQL query followed by an XSLT transformation on SPARQL's query results XML format [23]. The advantage of such a two-step approach is first that since SPARQL works on the RDF data model, all the input data from Fig. 2 are considered to be equivalent. Second, if one deploys a SPARQL engine that supports RDFS inference also

\footnotetext{
${ }^{3}$ http://vocab.org/relationship/.
} 
input data that involves ontologically related RDF vocabularies could be dealt with. For example, to get all persons who work with (rel:worksWith) or have met (rel:hasMet) Charles from the FOAF data described in Fig. 5, a simple SPARQL query would be enough:

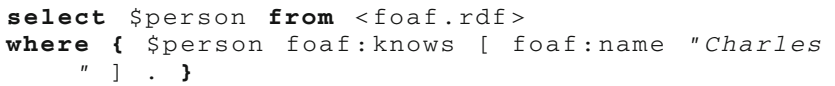

Although the approach proposed by the SAWSDL Working Group provides a good starting point, we argue that it can still be improved on several points: first, the detour through SPARQL's XML query results format seems to be an unnecessary burden. Second, a more tightly coupled integration of SPARQL and XML query languages can provide a more expressive language, beyond the capabilities of using SPARQL and XSLT or XQuery sequentially, and directly amenable to query optimisations. XSPARQL, the language proposed in the present paper, aims to provide exactly this: use cases that otherwise would require interleaved calls to SPARQL (typically requiring an implementation using an external programming framework) can be solved in XSPARQL directly, cf. the lowering example in Fig. 10. Moreover, as we will see, the combined language not only allows for concise lifting and lowering, but also may be viewed as an extension of SPARQL for RDF-to-RDF transformations, cf. the example in Fig. 8b below. Before we turn to these examples and XSPARQL in more detail, let us give a short overview of the languages XSPARQL builds on: XQuery and SPARQL.

\section{Preliminaries}

XQuery allows for a convenient and concise syntax for XML query processing and XML transformation, while SPARQL is the standard for RDF querying and transformation. One of the major differences between XQuery and SPARQL resides on the ordering of their respective data models: while XML (and hence XQuery) is an intrinsically ordered data model, RDF is an unordered data model. As such, necessary mechanisms must be in place to ensure XQuery respects the ordering of the input. Queries in each of the two languages can roughly be divided in two parts: (i) the retrieval part (body) and (ii) the result construction part (head): this is presented schematically in Fig. 6. Our goal is to combine these components for both languages in a unified language, XSPARQL, where XQuery's and SPARQL's heads and bodies may be used interchangeably and even nested. We next outline some of the main aspects of XQuery and SPARQL relevant to their combination into XSPARQL. For a more detailed overview of XQuery and SPARQL we refer the reader to [22,27] and to $[51,54]$.

\begin{tabular}{l|l|l|}
\hline \multirow{2}{*}{ Prolog: } & $\mathbf{P}$ & $\begin{array}{l}\text { declare namespace } \\
\text { prefix="namespace-URI" }\end{array}$ \\
& $\mathbf{F}$ & for var $[$ at posVar] in FLWOR expression \\
Body: & $\mathbf{L}$ & let var $:=$ FLWOR expression \\
& $\mathbf{W}$ & where FLWOR expression \\
$\mathbf{O}$ & order by FLWOR expression \\
Head: & $\mathbf{R}$ & return XML+ nested FLWOR expressions \\
\cline { 2 - 3 } & &
\end{tabular}

(a) Simplified schematic view on XQuery

\begin{tabular}{|c|c|c|}
\hline Prolog & $\mathbf{P}$ & prefix prefix: <namespace-URI> \\
\hline Head: & $\overline{\mathrm{C}}$ & Construct $\{$ template $\}$ \\
\hline Body: & $\begin{array}{l}\mathbf{D} \\
\mathbf{W} \\
\mathbf{M}\end{array}$ & $\begin{array}{l}\text { from / from named }<\text { dataset-URI }> \\
\text { where }\{\text { graph pattern }\} \\
\text { order by expression } \\
\text { limit integer }>0 \\
\text { offset integer }>0\end{array}$ \\
\hline
\end{tabular}

(b) Simplified schematic view on SPARQL

Fig. 6 An overview of XQuery and SPARQL

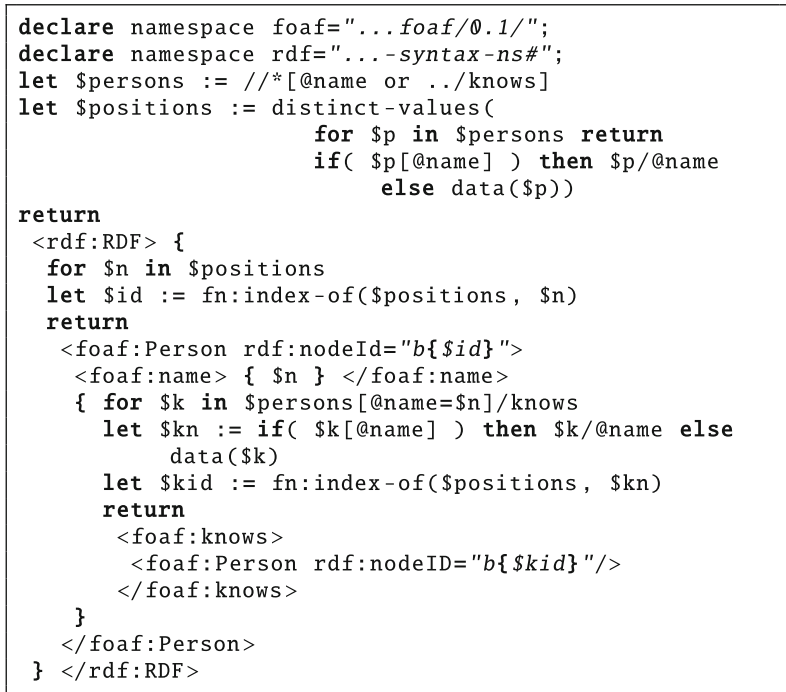

Fig. 7 Lifting using XQuery

\subsection{XQuery}

XQuery consists mainly of so-called FLWOR expressions, denoting the body (FLWO) and the head (R) of a query. The ForClauses $(\mathbf{F})$ can be used to declare variables that iterate over XML sequences, returned, e.g., by an XPath expression, while let assignments (L) allow to bind values, e.g., the entire result of an XPath expression, to variables. A filter condition on the current variable bindings or processing order of results within a ForClause can be specified in the where part $(\mathbf{W})$ and by the order by clause $(\mathbf{O})$, respectively. In the head $(\mathbf{R})$ arbitrary well-formed XML, nested XQuery expressions, or previously assigned variables are allowed following the return keyword. Together with a large catalogue of built-in functions [45], XQuery offers a flexible instrument for arbitrary XML transformations. 
The lifting task of Fig. 1 can be solved with XQuery as shown in Fig. 7. ${ }^{4}$ Please note that, due to the nature of XQuery, in this query we are generating RDF/XML, opposed to the more concise Turtle syntax from Fig. 1. The resulting query is quite involved, but completely addresses the lifting task, including unique blank node generation for each person. We first select, in variable \$persons (line 3), all nodes representing person names: either person or knows nodes and next, for each different name, we keep a sequence with all the distinct person names (stored in variable \$positions), which we will use as the blank node identifier for the person. Iterating over these distinct person names, we determine the person identifier (line 10). The nested for (lines 14-23) again iterates over persons in order to create nested foaf: knows elements: for each person name $(\$ n)$ from the outer for expression, this nested expression selects the XML nodes that correspond to persons which $\$ n$ knows (line 14) and creates the corresponding foaf : knows elements (lines 18-20). While this is a valid solution for lifting, we still observe the following drawbacks: (1) We still have to build RDF/XML manually and cannot make use of the more readable and concise Turtle syntax; and (2) if we had to apply XQuery for the lowering task, we still would need to cater for all kinds of different RDF/XML representations. Thus we still face the same problems as discussed in the XSLT solution in Sect. 2. However, both these drawbacks will be alleviated by adding SPARQL to XQuery. By combining XQuery and SPARQL, XSPARQL also simplifies the lifting process by allowing to use SPARQL ConstructClauses that generate RDF in Turtle format and by performing automatic validation of the generated RDF graphs.

Semantics. Next, let us give a short overview of the XQuery Formal Semantics [27], on which we will base XSPARQL's semantics; it is defined essentially via three types of rules: (i) normalisation rules, (ii) static typing rules, and (iii) dynamic evaluation rules. Normalisation rules are used to rewrite arbitrary XQuery expression to the XQuery Core language - a subset of XQuery that, while semantically equivalent, aims to be easier to define, implement, and optimise [41]. Static typing rules are used to assign a type to each XQuery expression, while the dynamic evaluation rules are responsible for producing the resulting XML from each expression and guaranteeing that the expression input is consistent with the typing information determined during the static analysis step. Any XQuery expression $E$ is evaluated with regard to an expression context $C$ that holds the static environment (statEnv) and the dynamic environment (dynEnv) up until the evaluation of $E$. Environments are composed of different components and hold information necessary to the evaluation of any XQuery expression: statEnv

\footnotetext{
${ }^{4}$ We assume this query is executed with the context item set externally to the document node of relations.xml file presented in Fig. 1.
}

holds the information available during static analysis, for example the varType component holds variable type information, while the dynEnv environment contains information available during expression evaluation, like the value for variables that is stored in the varValue component. We refer to the static environment of $C$ as $\operatorname{stat} \operatorname{Env}(C)$ and to the dynamic environment as dynEnv $(C)$ and we can access the different components by name: $\operatorname{statEnv}(C)$.varType and the specific value for element var of the a context can be accessed using statEnv $(C)$.varType(var). In case the expression context $C$ is not explicitly presented, statEnv and dynEnv can be used in place of statEnv $(C)$ and $\operatorname{dynEnv}(C)$.

Normalisation rules are represented using mapping rules and, as an example, we present the following rule from Draper et al. [27] that illustrates the normalisation of consecutive ForClauses into XQuery Core:

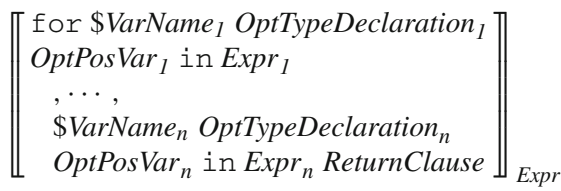

$$
\begin{aligned}
& == \\
& \text { for \$VarName } \text { OptTypeDeclaration }_{1} \\
& \text { OptPosVar }_{1} \text { in } \llbracket \operatorname{Expr}_{1} \rrbracket_{\text {Expr }} \text { return } \\
& \text {.. } \\
& \text { for \$ VarNamen } \text { OptTypeDeclaration }_{n} \\
& \text { OptPosVar }_{n} \text { in } \llbracket \text { Expr }_{n} \rrbracket_{\text {Expr }} \llbracket \text { ReturnClause } \rrbracket_{\text {Expr }}
\end{aligned}
$$

In normalisation rules, fixed-width font (like for) refers to specific keywords, and italic font refers to productions in the XQuery Core grammar [27, Appendix A]. Static type rules, and dynamic evaluation rules are represented using inference rules. For instance, the following static typing rule from Draper et al. [27] ensures that no expression has empty type except the empty sequence and functions in the $f s$ namespace that are applied to empty parentheses ():

$$
\begin{gathered}
\text { statEnv } \vdash \text { Expr : Type } \\
\text { statEnv } \vdash \text { Type <: empty } \\
\frac{\operatorname{not}\left(\begin{array}{l}
\text { Expr is the empty parentheses () or } \text { fn:data }() \\
\text { or any } f_{s} \text { function applied to empty parentheses () }
\end{array}\right)}{\text { A static type error is raised for expression Expr }}
\end{gathered}
$$

The judgements statEnv $\vdash$ Expr : Type and statEnv $\vdash$ Type $<$ : empty hold when, in the static environment statEnv, both Expr has type Type and Type is a subtype of empty, respectively. For all details of the XQuery Semantics we refer the reader to [27].

Typing Fernández et al. [30] describe the XQuery and XPath Data Model (XDM) that is used to define the input to XQuery and the values of any XPath expression. Draper et al. [27, Section 2.4] describe the formal notation for types that we use throughout this paper. This representation of types is used for specification purposes only and is not exposed to the end user by XQuery. As [27, Section 8.3.1] describe, it 
is possible to match a Value against a specific Type by using the judgement Value matches Type.

\subsection{SPARQL}

In analogy to FLWOR in XQuery, we will denote its correspondent "DWMC expressions" in SPARQL. The body (DWM) offers the following features: a dataset (D), i.e., the set of (named) source RDF graphs, is specified in from (or from named) clauses. The where part (W) allows matching parts of the dataset by specifying a graph pattern. Such patterns can be simple triple patterns possibly involving variables, URI references, and literals, ${ }^{5}$ or unions of graph patterns, optional patterns matching of parts of a graph, or patterns matching of named graphs, etc.

Definition 2 (Graph Patterns, [51]) Let $\mathbb{V}$ be an infinite set of variables, graph patterns are inductively defined as follows:

- a tuple $(s, p, o) \in \mathbb{U L} \mathbb{V} \times \mathbb{U} \mathbb{V} \times \mathbb{U} \mathbb{L} \mathbb{V}$, called triple pattern, is a graph pattern;

- a set of triple patterns, called Basic Graph Pattern (BGP), is a graph pattern;

- if $P$ and $P^{\prime}$ are graph patterns, then $\left(P P^{\prime}\right),(P$ optional $\left.P^{\prime}\right)$, and ( $P$ union $\left.P^{\prime}\right)$ are graph patterns;

- if $P$ is a graph pattern and $i \in \mathbb{U V}$, then (graph $i P$ ) is a graph pattern; and

- if $P$ is a graph pattern and $R$ is a filter expression, then $(P$ filter $R)$ is a graph pattern.

For any pattern $P$, we write $\operatorname{vars}(P)$ for the set of all variables occurring in $P$. A filter expression $R$ can be composed from constants, elements of $\mathbb{U L} \mathbb{V}$, comparison opera-

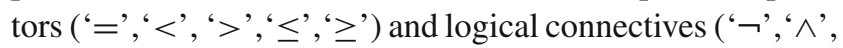
' $\vee$ '), and built-in functions. ${ }^{6}$

The evaluation semantics of SPARQL consists of computing a sequence of solution mappings, i.e., sets of bindings for the variables in these patterns, matching them against the graphs in the dataset. Sequences of solution mappings as referred to simply as solution sequences.

SPARQL is agnostic to the actual XML representation of the underlying source graphs, which alleviates the pain of having to deal with different RDF/XML representations of the graphs in the dataset. Also several RDF source graphs [39]

\footnotetext{
$\overline{5}$ Note that we do not allow blank nodes in graph patterns, and thus do not consider them in our definitions. This restriction does not affect the expressivity of SPARQL, implicit in [51], since blank nodes in query patterns can always be replaced equivalently with variables. See discussion in Sect. 4.2 below.

${ }^{6}$ For a complete list of built-in functions we refer the reader to [54].
}

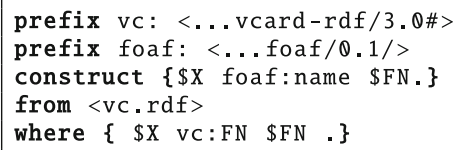

(a) Mapping full names from vCard to FOAF in SPARQL

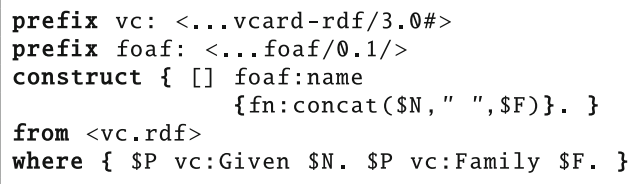

(b) Mapping a given and family name to a full name in XSPARQL

Fig. 8 RDF-to-RDF mappings in SPARQL and in XSPARQL

specified in consecutive from clauses can be merged transparently in SPARQL, however for XML tools this involves renaming of blank nodes at the pure XML level. Solution sequences can be ordered or sliced using solution modifiers (M) order by, limit, and offset.

In the head, SPARQL's construct clause (C) offers convenient and XML-independent means to create an output RDF graph. A construct template consists of a list of triple patterns in Turtle syntax. By instantiating this template with the variable bindings computed in the body, a result graph is created, which enables SPARQL to be used as a transformation language between different RDF formats (similar to XSLT and XQuery for transforming between XML formats). A simple example for mapping full names from the $\mathrm{vCard} / \mathrm{RDF}$ [40] format to foaf: name is given by the SPARQL query in Fig. 8. Blank nodes in construct templates-as used in the query in Fig. 8b-play a special role, in that they are replaced by a fresh blank node for each solution sequence in the result graph.

Other possible types of SPARQL queries include select, ask, and describe queries: select queries simply return the bindings for variables present in the query (instead of using these bindings to instantiate the template like a construct clause), ask queries return a boolean answer, indicating whether the graph pattern produces any results, and describe queries are used to return information about a resource. For the aims of XSPARQL we support the ConstructClause that allows to produce RDF and the select expression that allows us to input RDF data-although with a different syntax as explained in Sect. 4.1.

Let us remark that SPARQL does not cater for the creation of new values, which on the contrary is an inherent feature of XQuery. By combining XQuery and SPARQL, we are also enabling SPARQL to use the full range of XPath/XQuery built-in functions [45].

Due to this, the query in Fig. 8 which attempts to merge family names and given names into a single foaf : name by 
calling the $\mathrm{fn}$ : concat function is beyond SPARQL's capabilities. As we will see, XSPARQL will not only reuse SPARQL for transformations from and to RDF, but also enable such advanced RDF-to-RDF transformations.

Semantics The semantics of SPARQL is defined by means of evaluation rules which are presented by [54, Section 12.5]. Here we only give an overview of the notion of Basic Graph Pattern (BGP) matching that we will use later to define the semantics of XSPARQL.

The matching of BGPs is done with regard to a specific $\mathrm{RDF}$ graph - the active graph - which is a graph contained in the dataset specified to the query. This matching is defined in terms of replacing variables from the BGP with RDF terms present in the active graph, where the function that maps query variables to RDF terms is called a solution mapping.

Definition 3 (Solution Mapping) A solution mapping [see 54, Section 12.1.6] is a partial function mapping SPARQL variables to RDF terms. The domain of a solution mapping $\mu$, denoted $\operatorname{dom}(\mu)$, is the set of variables for which $\mu$ is defined. Furthermore, we denote the value of variable $v \in \mathbb{V}$ according to solution $\mu$ as $\mu(v)$. Two solution mappings $\mu_{1}$ and $\mu_{2}$ are compatible if for any $v \in \operatorname{dom}\left(\mu_{1}\right) \cap \operatorname{dom}\left(\mu_{2}\right)$ it holds that $\mu_{1}(v)=\mu_{2}(v)$. The union of two compatible mappings $\mu_{1}$ and $\mu_{2}$ consists of the standard set-theoretical union $\mu_{1} \cup \mu_{2}$.

The replacement of variables in a graph pattern according to a solution mapping is defined next.

Definition 4 Let $P$ be a graph pattern and $\mu$ be a solution mapping. The variable substitution of $P$ by $\mu$, denoted $\mu(P)$, is the graph pattern $P$ with all variables $v \in \operatorname{vars}(P) \cap$ $\operatorname{dom}(\mu)$ substituted by $\mu(v)$.

Finally, the definition of BGP matching from [54, Section 12.3] specifies the solutions to a query.

Definition 5 (Basic Graph Pattern Matching) We say $\mu$ is a solution for a BGP $P$ with respect to the active graph $G$, if there exists a solution mapping $\mu^{\prime}$ such that $\mu^{\prime}(P)$ is a subgraph of $G$, and $\mu$ is the restriction of $\mu^{\prime}$ to the variables in $\operatorname{vars}(P)$.

The definition of BGP matching is extended to more complex SPARQL query patterns (including union, optional, graph, filter, etc.) by the SPARQL algebra [54, Section 12.4], such that the where clause of every SPARQL query-i.e., any DWM body-returns a list of solutions. We denote this evaluation of a SPARQL Graph Pattern $P$ over a dataset $D$ as $\operatorname{eval}(D, P)$. As presented by [51], the evaluation of a SPARQL graph pattern can be specified by mapping the graph pattern to relational algebra operators. Since Perez et al. deal with set-based semantics of SPARQL, here we extend their notion of the join operator to solution sequences. Let $\Omega_{1}$ and $\Omega_{2}$ be two solution sequences; then $\Omega_{1} \bowtie \Omega_{2}=\operatorname{ToList}\left(\left\{\mu_{1} \cup \mu_{2} \mid\left(\mu_{1}, \mu_{2}\right) \in\right.\right.$
$\operatorname{ToMultiSet}\left(\Omega_{1}\right) \times \operatorname{ToMultiSet}\left(\Omega_{2}\right), \mu_{1}$ and $\mu_{2}$ are compatible\}), where by $\times$ we denote the Cartesian product of the multisets and ToList() is, as per the SPARQL specification [cf. 54, Section 12.4], an operation that turns a multiset into a sequence with the same elements and arbitrary ordering. Analogously to the ToList () operation, ToMultiSet() converts a sequence into a multiset by preserving duplicates but disregarding the sequence ordering.

For further details on the SPARQL query language, we refer the reader to the $\mathrm{W} 3 \mathrm{C}$ specification [54].

Next, we define the notion of inclusion of solution sequences.

Definition 6 Let $\Omega_{1}$ and $\Omega_{2}$ be solution sequences. We say $\Omega_{1}$ is included in $\Omega_{2}$, denoted $\Omega_{1} \preceq \Omega_{2}$, if for all solution mappings $\mu_{1} \in \operatorname{ToMultiset}\left(\Omega_{1}\right)$ there exists a solution mapping $\mu_{2} \in \operatorname{ToMultiset}\left(\Omega_{2}\right)$ such that $\mu_{1} \subseteq \mu_{2}$.

Please note that this definition extends the notion of subset between multisets by considering also the subset relation between their elements, i.e., solution mappings. This definition will be required for the optimisations presented in Sect. 6. Since the presented optimisations are not order preserving we rely only on the notion of inclusion.

In a construct query, the solutions of the pattern in the where clause of the body are then used to instantiate the construct template and the result graph is obtained from the union of all valid RDF triples resulting from such instantiation. As mentioned before, for each solution, blank nodes occurring in a construct template are replaced by new blank nodes with new identifiers.

Apart from construct queries, which we mainly focus on here, SPARQL also allows select queries, which return sequences of variable bindings, obtained from projecting only solution mappings for a given list of variables.

\section{XSPARQL}

Conceptually, XSPARQL is a merge of SPARQL construct queries into XQuery. This combination of languages allows us to benefit from the facilities of SPARQL for retrieving RDF data and to use Turtle-like syntax for constructing RDF graphs, while still having access to all the features from XQuery for XML processing. In XSPARQL we allow any native XQuery query and we extend XQuery's FLWOR expressions to what we call FLWOR' expressions:

(i) In the body we allow SPARQL-style F'DWM blocks alternatively to XQuery's FLWO blocks. The new F' clause of the form forvarlist is very similar to XQuery's native ForClause, but instead of allowing a single variable (which is assigned to the results of an XPath expression), the new clause supports a white space-separated list of variables (varlist). Each variable in varlist 


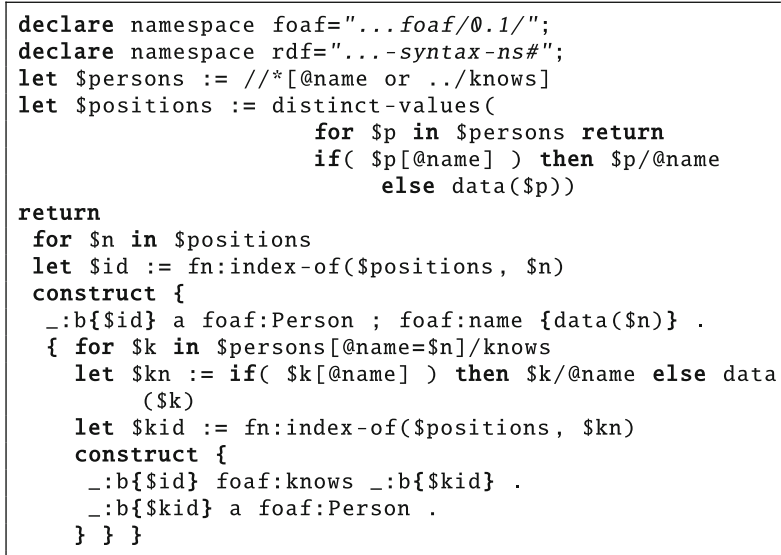

Fig. 9 Lifting in XSPARQL

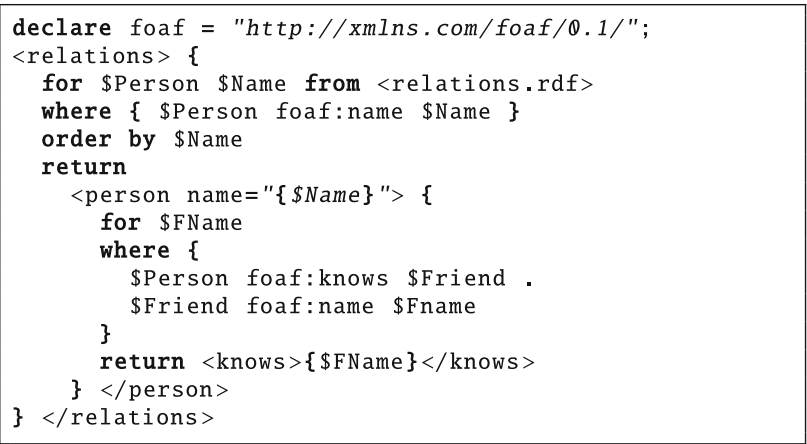

Fig. 10 Lowering using XSPARQL

is then assigned the value resulting from evaluating a SPARQL query of the form: selectvarlist DWM.

(ii) In the head we allow to create RDF graphs directly using construct templates (C) alternatively to XQuery's native return $(\mathbf{R})$.

(iii) Different forms of nesting are allowed, for example subqueries that construct RDF graphs may appear in let assignments which are later used in SPARQL-style from clauses, or can be used for value construction within SPARQL-style construct templates.

These modifications allow us to reformulate the lifting query of Fig. 7 on page 151 into its slightly more concise XSPARQL version of Fig. 9. The real power of XSPARQL in our example becomes apparent on the lowering part, where all of the other languages observed so far struggled. The lowering query for our running example is shown in Fig. 10.

\subsection{Syntax of XSPARQL}

In more detail, the XSPARQL syntax is an extension of the grammar rules in XQuery [22]. Figure 11 shows a schema of our merge of XQuery and SPARQL. For the definition of the XSPARQL syntax, we assume to inherit all the grammar productions of SPARQL [54] and XQuery [22] and mark any

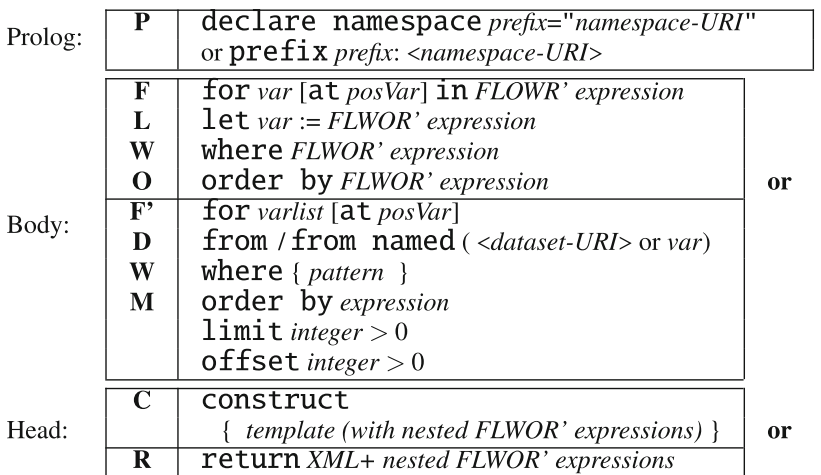

Fig. 11 Schematic view of XSPARQL

modified grammar productions with the prime symbol ('). We introduce two new productions: SparqlForClause and ConstructClause, corresponding to roughly to SPARQL select queries and construct templates; we present the grammar productions for these in Fig. 12. The full XSPARQL grammar can be found in [15]. In these grammar productions, the Where Clause and SolutionModifier correspond, respectively, to rules [13] and [14] from the SPARQL grammar, cf. [54, Appendix A.8].

The newly introduced SparqlForClause(rule [33a]) is similar to an XQuery for clause that can be used to iterate over SPARQL results. ${ }^{7}$ This expression stands at the same level as XQuery's for and let expressions, i.e., such type of clauses are allowed to start new FLWOR' expressions, or may occur inside deeply nested XSPARQL queries.

The ConstructTemplate' expression is defined in the same way as the production ConstructTemplate in SPARQL [54], but we additionally allow nested XSPARQL expressions (FLWORExpr') in subject, predicate, and object positions; we achieve this by replacing SPARQL syntax rules Verb and VarOrTerm for ConstructTemplate with the rules VarOrTerm' and Verb' represented in Fig. 12b. ${ }^{8}$

The rules for Sourceselector from the SPARQL syntax are also extended (as presented in Fig. 12c), i.e., we allow for graphs in a SPARQL dataset to be specified by a variable which must evaluate to a URI.

In analogy to SPARQL's select * shortcut, we allow to write for * in place of for [list of all unbound variables appearing in the WhereClause] for SparqlForClauses; as syntactic sugar this is also the default value for the $\mathbf{F}$ ' clause whenever a SPARQL-style WhereClause is found and a corresponding F' clause is missing. Please note that for SparqlForClauses we do not allow XQuery QNames as variable

\footnotetext{
7 This expression does not have the exact semantics of a SPARQL select clause - returning bindings to variables — but rather adds new variables to the query; hence a syntax inspired by the existing XQuery ForClauses was chosen.

8 These changes are highlighted in Fig. 12 by using bold face.
} 


\begin{tabular}{|lll|}
\hline [33] FLWORExpr' & $::=$ ((ForClause | LetClause)+ XQWhereClause? OrderByClause?) | SparqlForClause) \\
& ("return" ExprSingle | ConstructClause) \\
[33a] SparqlForClause & $::=$ "for" (VarName+ | "*") DatasetClause? WhereClause? SolutionModifier \\
[33b] ConstructClause & $::=$ "construct" ConstructTemplate' \\
\hline
\end{tabular}

(a) XSPARQL core syntax elements, extending [22, Appendix A]

$\begin{array}{ll}\text { [37] Verb' } & ::=\text { VarOrIRIref | "a" | "\{" FLWORExpr' " }\} \text { " } \\ \text { [42] VarOrTerm, } & ::=\text { Var | GraphTerm | "\{" FLWORExpr' "\}" }\end{array}$

(b) Modified ConstructTemplate syntax elements, extending [54, Appendix A]

[12] SourceSelector' ::= IRIref | "\$" VarName

(c) Modified DatasetClause syntax elements, extending [54, Appendix A]

Fig. 12 Overview of XSPARQL syntax

names (further details are available in [27, Section 3.1.1.1]) and assume that only unprefixed variables are shared between the XQuery and SPARQL expressions of XSPARQL. By this treatment, XSPARQL becomes a syntactic superset of native SPARQL construct queries, since we additionally allow the following:

(1) XQuery and SPARQL namespace declarations (P) may be used interchangeably; and

(2) SPARQL-style construct result forms (C) may appear before the retrieval part for queries. This feature is mainly added in order to encompass SPARQL style queries, but in principle, we expect the $(\mathbf{R} / \mathbf{C})$ parts to appear in the end of a FLWOR' expression.

Thus, the query of Fig. 8a on page 153 or any other SPARQL construct queries remain valid syntax for XSPARQL.

\subsection{Semantics of XSPARQL}

Next we define the semantics of XSPARQL. After introducing some new types, used in the semantics, and an extension to the normalisation rules of XQuery ForClauses, we will turn to extending the notion of Basic Graph Pattern matching (Sect. 4.2) to make SPARQL clauses aware of the bindings for variables from XQuery. Then, we present the semantics of the newly introduced expressions: SparqlForClause(Sect. 4.2) and ConstructClause (Sect. 4.2), based on XQuery's formal semantics [27], by defining normalisation, static type and dynamic evaluation rules for each of the new expressions.

XSPARQL Types. We extend the XQuery and XPath Data Model (XDM), described by Fernández et al. [30], with the following new types that accommodate for SPARQL specific parts of XSPARQL:

(1) the RDFTerm type further consists of the subtypes uri, bnode and literal and is used as the type of SPARQL variables;

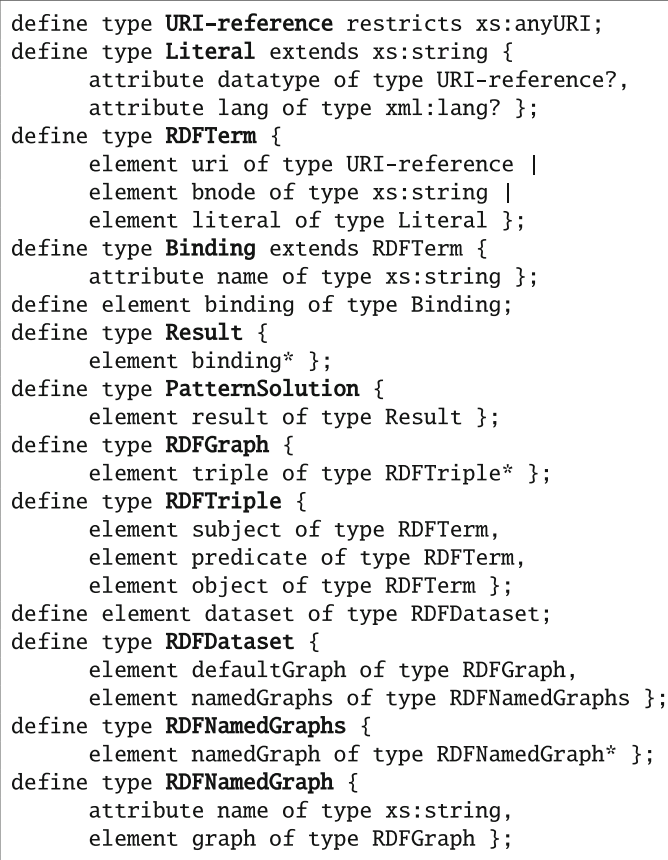

Fig. 13 XSPARQL Type Definitions

(2) the PatternSolution type consists of a set of pairs (variableName, RDFTerm) representing SPARQL variable bindings;

(3) the RDFGraph is the type of construct expressions; and

(4) the RDFDataset as the type for DatasetClauses.

The formal definition of (1)-(4) is given in Fig. 13. The RDFTerm type is used to represent RDF terms (composed of URIs, blank nodes or literals). The type of SPARQL variables are represented by the Binding type, that consists of the variable name and the RDF term that is assigned to it. Finally, sequences of SPARQL variable bindings are represented by the type PatternSolution. This representation of SPARQL results is similar to the XML Schema of the SPARQL Query 
Results XML Format, available at http://www.w3.org/2007/ SPARQL/result.xsd.

The RDFGraph type corresponds to a sequence of RDFTriples which are in turn a complex type composed of subject, predicate and object. The RDFDataset type is defined as an RDFGraph that is considered the default graph and a sequence of RDFNamedGraphs represented by the name of the graph and the corresponding RDFGraph.

The following definition presents the translation between a SPARQL solution sequence and a sequence of Result type elements that we implement in XSPARQL.

Definition 7 (Serialisation of Solution Sequences) Given a solution sequence $\Omega=\left(\mu_{1}, \ldots, \mu_{n}\right)$ a serialisation of $\Omega$ into a sequence of PatternSolution is defined as follows:

$$
\begin{aligned}
& -\operatorname{serialise}(\Omega) \Rightarrow \operatorname{serialise}\left(\mu_{1}\right), \ldots, \operatorname{serialise}\left(\mu_{n}\right) \\
& <\text { result }> \\
& -\operatorname{serialise}(\mu) \Rightarrow \quad\{\forall x \in \operatorname{dom}(\mu), \operatorname{serialise}(\mu, x)\} \\
& </ \text { result }> \\
& <\text { binding name }=" x "> \\
& -\operatorname{serialise}(\mu, x) \Rightarrow \quad\{\operatorname{term}(\mu(x))\} \quad \text {, } \\
& </ \text { binding }>
\end{aligned}
$$

where $\operatorname{term}(\mu(x))$ is

$$
\begin{aligned}
& -<\operatorname{uri}>\mu(x)</ \text { uri }> \\
& -<\text { bnode }>\mu(x)</ \text { bnode } \\
& -<\text { literal }>\mu(x)</ \text { literal }>
\end{aligned}
$$

Following the definition of the serialise function, in evaluation rules, we will refer to sequences of elements of type PatternSolution as $\Omega$ and to elements of type Result as $\mu$.

Query Prolog Normalisation As stated previously, XQuery and SPARQL namespace declarations can be used interchangeably in the query prolog. Hence, we convert any SPARQL syntax prefix declaration to XQuery namespace declarations by the following normalisation rules:

$$
\begin{aligned}
& \text { 【prefix NCName : }\langle\text { URILiteral }\rangle \rrbracket_{\text {Expr }} \\
& == \\
& \text { ₫declare namespace NCName = URILiteral } ; \rrbracket_{\text {Expr }} \\
& \text { 【prefix : }<\text { URILiteral }>\rrbracket_{\text {Expr }} \\
& == \\
& \llbracket \text { declare default namespace }=\text { URILiteral } ; \rrbracket_{\text {Expr }} \\
& \text { 〔base }<\text { URILiteral }>\rrbracket_{\text {Expr }} \\
& == \\
& \text { 【declare base-uri URILiteral ; } \rrbracket_{\text {Expr }}
\end{aligned}
$$

XQuery for normalisation In accordance with the SPARQL semantics, blank nodes in ConstructTemplates need to be distinctly instantiated for any solution mapping matching the body, i.e., for every solution for the WhereClause a new blank node identifier needs to be created in the resulting graph. To ensure this behaviour in XSP-
ARQL ConstructTemplates, we will use position variables ${ }^{9}$ from XQuery in ForClauses to generate these new blank node identifiers, i.e., we introduce position variables in any XQuery for expressions without position variables and also to make sure that XSPARQL SparqlForClauseexpressions have position variables. To handle the XQuery for expression, we change the normalisation rule of for expressions to XQuery Core for expressions (cf. Sect. 3.1):

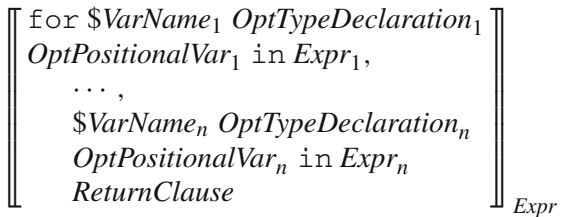

$$
\begin{aligned}
& == \\
& \text { for \$VarName } \text { OptTypeDeclaration }_{1} \\
& \llbracket O p t P o s i t i o n a l V a r_{1} \rrbracket_{\text {PosVar }} \text { in } \llbracket \operatorname{Expr}_{1} \rrbracket_{\text {Expr }} \\
& \text { return } \\
& \text {... } \\
& \text { for \$VarName } \text { OptTypeDeclaration }_{n} \\
& \llbracket \text { OptPositionalVar }_{n} \rrbracket_{\text {PosVar }} \text { in } \llbracket \text { Expr }_{n} \rrbracket_{\text {Expr }} \\
& \text { 【ReturnClause } \rrbracket_{\text {Expr }}
\end{aligned}
$$

A new normalisation rule $\llbracket \cdot \rrbracket_{\text {PosVar }}$ takes care of introducing new positional variables where necessary. We assume that the introduced position variables are distinct from any of the variables in scope, represented by the formal semantics variable $\$$ fs:new [cf. 27, Section 4.12.6]: $\llbracket \mathbb{D}_{\text {Pos Var }}==$ at $\$$ s:new. In case a positional variable is already present it is reused: $\llbracket$ at $\$$ PosVar $\rrbracket_{\text {PosVar }}==$ at $\$$ PosVar.

We also assume a new static environment component statEnv.posVars which consists of a sequence holding all positional variables in the given static environment, that is, the variables defined in the at clause of enclosing for expressions. The static type rules for the for expression [cf. 27, Section 4.8.2] need to be extended accordingly to store these positional variables, similar to the rules for SparqlForClauses in Sect. 4.2 below.

XSPARQL BGP Matching In this section we extend the notion of Basic Graph Pattern (BGP) matching described by [54, Section 12.3], to provide SPARQL with the variable bindings from XQuery. For this we rely on the XQuery varValue dynamic environment component that maps variable names to their value and consider this environment component as defining a set of bindings in the spirit of SPARQL solution mappings (as presented in Definition 3). Along these lines, we will consider the varValue component of the dynamic environment in which a SPARQL graph pattern $P$ is executed the basis for the XSPARQL instance mapping of $P$. The transformation from the dynEnv.varValue into the XSPARQL instance mapping is defined next:

\footnotetext{
${ }^{9}$ Position variables are variables that appear in an XQuery ForClauses after the optional at keyword - cf. Fig. 6a on page 151 - and bind to an integer indicating the current position in the for-expression.
} 
Definition 8 (XSPARQL instance mapping) Let $C$ be an expression context, and $D_{C}=\operatorname{dynEnv}(C)$.varValue be the varValue and $T_{C}=\operatorname{statEnv}(C)$.varType be the varType component of the static environment of $C$, respectively. The XSPARQL instance mapping $\mu_{C}$ is a solution mapping where, for each mapping $v_{i} \rightarrow x_{i} \in D_{C}, x_{i}$ is converted into an instance of type RDFTerm or an RDF Collection according to the following conditions:

- if $x_{i}=()$ and $T_{C} \cdot \operatorname{varType}\left(v_{i}\right)=$ RDFTerm then $\mu_{C}\left(x_{i}\right)$ is undefined;

- if $x_{i}=()$ and $T_{C} \cdot \operatorname{varType}\left(v_{i}\right) \neq$ RDFTerm then $\mu_{C}\left(x_{i}\right)=$ () is an empty RDF Collection;

- if $x_{i}$ is a singleton sequence, then $\mu_{C}\left(x_{i}\right)=\operatorname{RDFTerm}\left(x_{i}\right)$;

- if $x_{i}=\left(e_{1}, \ldots, e_{n}\right), n>1$, is a sequence then $\mu_{C}\left(x_{i}\right)=$ $\left(\right.$ RDFTerm $\left(e_{1}\right) \cdots$ RDFTerm $\left.\left(e_{n}\right)\right)$ to be read as an RDF Collection [46, Section 4.2] in Turtle notation [see 7, Section 3.5];

where $\operatorname{RDFTerm}\left(x_{i}\right)$ is

$$
\begin{aligned}
& -x_{i} \quad \text { if } T_{C} \cdot \operatorname{varType}\left(v_{i}\right)=\text { RDFTerm, } \\
& \text { - " } x_{i} " \quad \text { if } T_{C} \cdot \operatorname{varType}\left(v_{i}\right)=\text { xsd:string, } \\
& \text { - " } x_{i} " \wedge \wedge \text { rdf:XMLLiteral if } T_{C} \cdot \operatorname{varType}\left(v_{i}\right)= \\
& \text { element (), } \\
& \text { - "data }\left(x_{i}\right) " \quad \text { if } T_{C} \cdot \operatorname{varType}\left(v_{i}\right)=\operatorname{attribute(),\text {and}} \\
& \text { - " } x_{i} " \wedge \wedge T_{C} \cdot \operatorname{varType}\left(v_{i}\right) \\
& \text { otherwise. }
\end{aligned}
$$

For a graph pattern $P$, we call the XSPARQL instance mapping of the expression context in which $P$ is executed the $X S P A R Q L$ instance mapping of $P$.

Next we define the notion of XSPARQL BGP matching based on the semantics of SPARQL BGP matching presented in Sect. 3.2.

Definition 9 (Extended solution mapping) Let $C$ be an expression context. An extended solution mapping of a graph pattern $P$ in $C$ is a solution mapping compatible with the $X S P A R Q L$ instance mapping of $C$.

XSPARQL BGP matching is defined analogously to the SPARQL BGP matching with the exception that we consider only extended solution mappings:

Definition 10 (XSPARQL BGP matching) Let $P$ be a basic graph pattern, $C$ be the expression context of $P$, and $G$ be an RDF graph. We say that $\mu$ is a solution for $P$ with respect to active graph $G$, if there exists an extended solution mapping $\mu^{\prime}$ of $C$ such that $\mu^{\prime}(P)$ is a subgraph of $G$ and $\mu$ is the restriction of $\mu^{\prime}$ to the variables in $\operatorname{vars}(P)$.

This definition quasi injects the variable bindings inherited from XQuery into SPARQL patterns occurring within XSPARQL; by considering extended solution mappings the bindings returned for a BGP $P$ will not only match the input graph
$G$ but also respect any bindings for variables in the dynamic environment. We can extend the XSPARQL BGP matching to generic graph patterns by following the SPARQL evaluation semantics, as described by [54, Section 12.4]. Considering a graph pattern $P$ and $\mu_{C}$ the XSPARQL instance mapping of $P$, we similarly denote by $\operatorname{eval}_{x S}\left(D, P, \mu_{C}\right)$ the evaluation of $P$ over dataset $D$ following XSPARQL BGP matching.

Matching blank nodes in nested queries As for the handling of explicit DatasetClauses we briefly review the scoping graph concept from SPARQL's semantics, presented in [54, Section 12]. Query solutions are taken from the scoping graph, a graph that is equivalent to the active graph but does not share any blank nodes with it or any graph pattern within the query. Although in XSPARQL we are not considering blank nodes in graph patterns, in the presence of nested SparqlForClauses XSPARQL instance mappings may in fact contain assignments of variables to blank nodes, injected from the outer SparqlForClause into the inner SparqlForClause. For example, in Fig. 10 on page 155, blank nodes bound in the outer SparqlForClause to the variable \$Person will be injected into the inner SparqlForClauseexpression. In XSPARQL-as opposed to SPARQL patterns-such injected bnodes will be matched like constants against the blank nodes from the data, to enable coreference within nested queries over the same dataset. To ensure this behaviour, we introduce the notion of active dataset; nested queries over the same active dataset keep the same the scoping graphs. Any SparqlForClause with an explicit DatasetClause causes the active dataset to change, i.e., new scoping graphs (with fresh blank nodes) for each graph within it are created; if no DatasetClause is present in a nested SparqlForClause (implicit dataset), the active dataset remains unchanged.

We introduce another auxiliary function in the XSPARQL semantics, $f$ s:dataset (DatasetClause), which returns an element of type RDFDataset based on the evaluation of its argument. This conversion is performed according to the SPARQL semantics presented in Sect. 3.2 and detailed in [54]. The static type signature of this function is

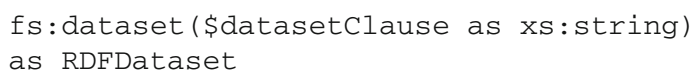

We allow the Sourceselector of a DatasetClause to be specified by an element of type uri or RDFGraph. Elements of the type uri in the position of a graph will be mapped to graphs where the uri is used as its name. XSPARQL - just like the SPARQL specification-leaves the exact mapping of URIs to graphs open to particular implementations, but for the rest of this paper, we assume obtaining the RDF graph just by dereferencing the URI via HTTP. 
SparqlForClause and XQuery ForClauses The semantics of SparqlForClause (Rule [33a], Fig. 12a) is defined by the following normalisation rules, static type analysis rules, and dynamic evaluation rules. We will also need to slightly adapt the static analysis rules for regular ForClauses (in order to properly deal with the extra position variables introduced by rule (N5)). We start with normalisation rules for SparqlForClauses with implicit variable selection (by means of "for *") and with explicitly stated variables:

$$
\begin{aligned}
& \llbracket \begin{array}{l}
\text { for * OptDatasetClause WhereClause } \\
\text { SolutionModifier return ExprSingle }
\end{array} \|_{\text {Expr }} \\
& = \\
& \text { [for } \llbracket \text { WhereClause } \rrbracket_{\text {vars }} \\
& \text { OptDatasetClause WhereClause } \\
& \text { \SolutionModifier return ExprSingle } \rrbracket_{\text {Expr }}
\end{aligned}
$$

The normalisation rule «WhereClause $\rrbracket_{\text {vars }}$ determines all statically unbound variables present in the WhereClause, i.e., returns a whitespace separated list of all variables in the WhereClause that are not present in the statEnv.varType environment component. The next normalisation rule introduces a new position variable, analogously to the beforementioned XQuery for normalisation rule, where $\llbracket \cdot \rrbracket_{\text {Pos Var }}$ is as described above:

$$
\begin{aligned}
& \text { Ifor } \$ \text { VarName }_{1} \ldots \text {... \$VarName } \\
& \text { OptDatasetClause WhereClause } \\
& \text { SolutionModifier return ExprSingle } \prod_{\text {Expr }} \\
& == \\
& \text { for } \$ \text { VarName }_{1} \ldots \text {... } \text { VarName }_{n} \llbracket \rrbracket_{\text {PosVar }} \\
& \text { OptDatasetClause WhereClause } \\
& \text { 【return ExprSingle } \rrbracket_{\text {Expr }}
\end{aligned}
$$

Static type analysis The following static rule takes care of defining the types of variables present in a for expression as RDFTerm, adds the introduced position variables to statEnv.pos Vars, and determines the static type of the SparqlForClause expression:

$$
\begin{aligned}
& \text { statEnv.posVars }=\left(\text { PosVar }_{1}, \cdots, \text { PosVar }_{n}\right) \\
& \text { statEnv } \vdash \text { PosVarName of var expands to PosVar } \\
& \text { statEnv + posVars }\left(\text { PosVar }_{1}, \cdots, \text { PosVar }_{n}, \text { PosVar }\right) \\
& +\operatorname{varType}\left(\begin{array}{l}
\text { PosVar } \Rightarrow \text { Xs: integer; } \\
\operatorname{Var}_{1} \Rightarrow \text { RDFTerm; } \\
\cdots ; \text { Var }_{n} \Rightarrow \text { RDFTerm }
\end{array}\right) \\
& \vdash \text { ExprSingle : Type } \\
& \text { for } \$ \operatorname{Var}_{1} \cdots \$ \operatorname{Var}_{n} \text { at PosVarName } \\
& \text { statEnv } \vdash \begin{array}{l}
\text { DatasetClause W } \\
\text { SolutionModifier }
\end{array} \\
& \text { return ExprSingle: Type* }
\end{aligned}
$$

Please note that, since the variables included in a SparqlForClause are not allowed to contain a namespace prefix, we omitted the rules handling the namespace expansion for the respective variables. The static type rule for a SparqlForClausewithout an explicit DatasetClause is analogous. Likewise, note that we need to slightly adapt the standard static type checking rules for standard XQuery [27, Section 4.8.2], to populate the XSPARQL specific new static environment component statEnv.pos Vars: ${ }^{10}$

$$
\begin{aligned}
& \text { statEnv.posVars }=\left(\text { PosVar }_{1}, \cdots, \text { PosVar }_{n}\right) \\
& \text { statEnv } \vdash \text { Expr }_{1}: \text { Type }_{1} \\
& \text { statEnv } \vdash \text { VarName of var expands to } V a r \\
& \text { statEnv } \vdash \text { VarName pos of var expands to } \text { Var }_{\text {pos }} \\
& \text { statEnv + posVars }\left(\text { PosVar }_{1}, \cdots, \text { Pos Var }_{n}, \text { Var }_{\text {pos }}\right) \\
& +\operatorname{varType}\left(\begin{array}{l}
\operatorname{Var} \Rightarrow \operatorname{prime}\left(\text { Type }_{1}\right) ; \\
\operatorname{Var}_{\text {pos }} \Rightarrow \mathrm{x} \text { :integer }
\end{array}\right) \\
& \vdash \text { ExprSingle : Type } \\
& \text { for \$VarName at \$VarName pos } \\
& \text { statEnv } \vdash \text { in Expr } r_{1} \text { return ExprSingle : } \\
& \text { Type } \cdot \text { quantifier }\left(\text { Type }_{1}\right)
\end{aligned}
$$

Dynamic Evaluation For the dynamic evaluation we have to introduce a new dynamic environment component called activeDataset that will be used to evaluate WhereClauses. Initially, this component is empty (or set to a system default) and is changed by a DatasetClause appearing in a SparqlForClause. We further introduce two auxiliary functions $f$ s:value and $f$ s:sparql.

fs:value The $f$ s:value( $\$ P S, \$ v a r)$ function returns the value of the specified SPARQL variable $\$$ var in a Pattern Solution specified by $\$ P S$. If $\$ v a r$ is not bound in $\$ P S$, the empty sequence is returned. This function is defined as

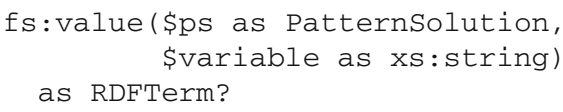

fs:sparql The $f_{s}$ :sparql function corresponds to the adapted version of the eval function, the $e v a l_{x s}$ function, that evaluates graph patterns implementing the extended notion of BGP Matching (cf. Definition 10).

The static type signature of this function is defined as

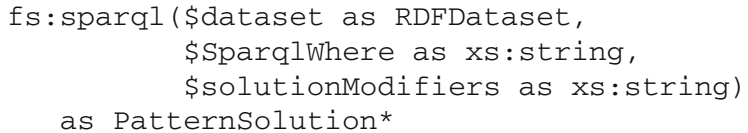

The parameters of the eval ${ }_{x s}$ function correspond to the dataset \$dataset, the SPARQL algebra expression generated from the graph pattern \$SparqlWhere and \$solutionModifiers, as described by [cf. 54, Section 12.2.3], and the XSPARQL instance mapping $\mu_{C}$ that is derived from the expression context $C$ over which the $f_{s}$ :sparql function is evaluated. The result of eval $x s$ consists of a solution sequence which, as a result of applying the serialise function (cf. Definition 7), can be translated directly into an XQuery sequence of XML elements of type Patternsolution.

We can now define the dynamic evaluation rules for the SparqlForClause expression. Intuitively, these rules state

\footnotetext{
${ }^{10}$ We show here only the adapted rule for ForClauses with position variables without type declaration, the rule handling both position variables and type declarations is adapted analogously.
} 
that the return expression ExprSingle will be executed for each Patternsolution that is returned from the evaluation of the $f$ s:sparql function. The following two dynamic rules specify the evaluation of the SparqlForClause with an explicit DatasetClause:

$$
\begin{aligned}
& \text { dynEnv } \vdash f \text { s:dataset (DatasetClause }) \Rightarrow \text { Dataset } \\
& \text { dynEnv } \vdash f \text { s:sparql }\left(\begin{array}{l}
\text { Dataset, WhereClause }, \\
\text { SolutionModifier }
\end{array}\right) \Rightarrow \mu_{1}, \ldots, \mu_{m} \\
& \text { dynEnv + activeDataset(Dataset) } \\
& +\operatorname{varValue}\left(\begin{array}{l}
\text { PosVar } \Rightarrow 1 \\
\operatorname{Var}_{1} \Rightarrow \text { fs:value }\left(\mu_{1}, \operatorname{Var}_{1}\right) ; \\
\ldots ; \\
\operatorname{Var}_{n} \Rightarrow \text { fs:value }\left(\mu_{1}, \operatorname{Var}_{n}\right)
\end{array}\right) \\
& \vdash \text { ExprSingle } \Rightarrow \text { Value }_{1} \\
& \text { : } \\
& \text { dynEnv }+ \text { activeDataset (Dataset) } \\
& +\operatorname{varValue}\left(\begin{array}{l}
\text { PosVar } \Rightarrow n ; \\
\operatorname{Var}_{1} \Rightarrow \text { ss:value }\left(\mu_{m}, \operatorname{Var}_{I}\right) ; \\
\ldots ; \\
\operatorname{Var}_{n} \Rightarrow \text { ss:value }\left(\mu_{m}, \operatorname{Var}_{n}\right)
\end{array}\right)
\end{aligned}
$$

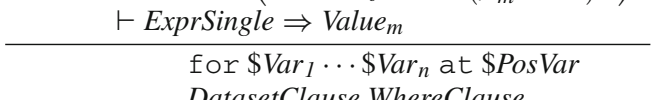

$$
\begin{aligned}
& \text { dynEnv } \vdash \begin{array}{l}
\text { DatasetClause WhereClause } \\
\text { SolutionModifier return }
\end{array} \\
& \text { ExprSingle } \Rightarrow \text { Value }_{1}, \ldots, \text { Value }_{m}
\end{aligned}
$$

This rule ensures that the activeDataset component of the dynamic environment is updated to reflect the explicit DatasetClause of the SparqlForClause. If the evaluation of the $f$ s:sparql function does not yield any solutions, i.e., evaluates to an empty sequence, the overall result will also be the empty sequence:

$$
\begin{aligned}
& \text { dynEnv.activeDataset } \Rightarrow \text { Dataset } \\
& \frac{\operatorname{dynEnv} \vdash f_{\text {s:sparql }}\left(\begin{array}{l}
\text { Dataset }, \text { WhereClause, } \\
\text { SolutionModifier }
\end{array}\right) \Rightarrow()}{\text { for } \$ \operatorname{Var}_{1} \cdots \$ \text { Var }_{n} \text { at } \$ \text { PosVar }} \\
& \text { dynEnv } \vdash \text { DatasetClause WhereClause } \Rightarrow(\text { ) } \\
& \text { SolutionModifier return ExprSingle }
\end{aligned}
$$

The rule that handles the SparqlForClause without an explicit DatasetClause is presented next:

$$
\begin{aligned}
& \text { dynEnv.activeDataset } \Rightarrow \text { Dataset } \\
& \text { dynEnv } \vdash f \text { s:sparql }\left(\begin{array}{l}
\text { Dataset, WhereClause, } \\
\text { SolutionModifier }
\end{array}\right) \Rightarrow \mu_{1}, \ldots, \mu_{m} \\
& \operatorname{dynEnv}+\operatorname{varValue}\left(\begin{array}{l}
\text { PosVar } \Rightarrow 1 ; \\
\operatorname{Var}_{1} \Rightarrow \text { ss:value }\left(\mu_{1}, \operatorname{Var}_{1}\right) ; \\
\ldots ; \\
\operatorname{Var}_{n} \Rightarrow \text { fs:value }\left(\mu_{1}, \operatorname{Var}_{n}\right)
\end{array}\right) \\
& \vdash \text { ExprSingle } \Rightarrow \text { Value }_{1} \\
& \operatorname{dynEnv}+\operatorname{varValue}\left(\begin{array}{l}
\text { PosVar } \Rightarrow n ; \\
\operatorname{Var}_{I} \Rightarrow f_{\text {s:value }}\left(\mu_{m}, \text { Var }_{I}\right) ; \\
\ldots ; \\
\operatorname{Var}_{n} \Rightarrow f_{\text {s:value }}\left(\mu_{m}, \text { Var }_{n}\right)
\end{array}\right) \\
& \vdash \text { ExprSingle } \Rightarrow \text { Value }_{m} \\
& \text { for } \$ \operatorname{Var}_{1} \cdots \$ \operatorname{Var}_{n} \text { at } \$ \text { PosVar } \\
& \text { dynEnv } \vdash \text { WhereClause SolutionModifier } \\
& \text { return ExprSingle } \Rightarrow \text { Value }_{1}, \ldots, \text { Value }_{m}
\end{aligned}
$$

Analogously to the SparqlForClause with an explicit dataset, whenever the $f$ s:sparql function evaluates to an empty sequence, the result will also be an empty sequence.

ConstructClause We now define the semantics of the ConstructClause (Rule [33b], Fig. 12a) by means of normalisation rules. SPARQL stand-alone construct queries (as described in Sect. 4.1) are normalised into construct queries with a surrounding ForClause:

$$
\begin{aligned}
& \left\|\begin{array}{l}
\text { Construct ConstructTemplate } \\
\text { DatasetClause WhereClause } \\
\text { SolutionModifier }
\end{array}\right\|_{\text {Expr }} \\
& =
\end{aligned}
$$

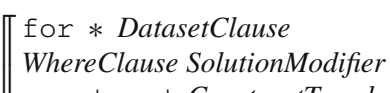

$$
\begin{aligned}
& \text { ¿construct ConstructTemplate' } \rrbracket_{\text {Expr }}
\end{aligned}
$$

The resulting query will be further rewritten according to aforementioned normalisation rule (N6). As introduced in Sect. 4.1, we allow nested XSPARQL expressions in subject, predicate, and object positions of ConstructTemplate'. These nested expressions are identified by the shortcuts $\{$ Expr $\}$, $<\{\operatorname{Exp} r\}>$, and $-:\{\operatorname{Expr}\}$, that construct elements of type literal, uri, and bnode, respectively.

Similar to the normalisation rule for stand-alone ReturnClauses presented in [27, Section 4.8.1], the following normalisation rule transforms construct clauses into XQuery return ExprSingle s.

$$
\begin{aligned}
& \text { 【construct ConstructTemplate' } \rrbracket_{\text {Expr }} \\
& \begin{array}{c}
== \\
\text { return } f \text { s:evalCT }\left(\llbracket \text { ConstructTemplate } \rrbracket_{\text {normCT }}\right)
\end{array}
\end{aligned}
$$

In the following we assume that ConstructTemplate' is a simple ". " separated list of Subject, Predicate, and Object. The $\llbracket \cdot \rrbracket_{\text {norm } C T}$ rule transforms any Turtle shortcut notation used in ConstructTemplate' to these simple lists. As an example of this rule, we present the rule for normalising Turtle ";" abbreviations [cf. 7, Section 2.3]:

$$
\begin{aligned}
\begin{aligned}
\llbracket \text { Subject Pred }_{1} \operatorname{Obj}_{1} & \ldots ; \text { Pred }_{n} \operatorname{Obj}_{n} \rrbracket_{n o r m C T} \\
& == \\
\text { Subject Pred } & \text { Obj }_{1} \ldots \text { Subject Pred }
\end{aligned} \text { Obj }_{n}
\end{aligned}
$$

The normalisation rules for the other Turtle shortcuts that are allowed in the SPARQL ConstructTemplate' syntax are similar to this one and are not presented here. Since anonymous blank nodes can be written in numerous ways in Turtle, the $\llbracket \cdot \rrbracket_{\text {normCT }}$ normalisation rule transforms each anonymous blank node into a labelled blank node where the identifier/label is distinct from any other blank node labels present in the ConstructTemplate'. Take, as an example, the ConstructTemplate in Fig. 8b on page 153. It is normalised as

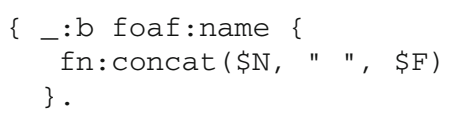


fs:evalCT The $f$ s:evalCT function is a new built-in function that ensures the created RDF graph is valid and rewrites any blank nodes inside of ConstructTemplates to comply with the SPARQL semantics (as described in Sect. 4.2). The auxiliary $f s$ :validTriple function checks if each triple is valid according to the RDF semantics and is defined by rules (D5) and (D6). The static type signatures of these functions are defined as

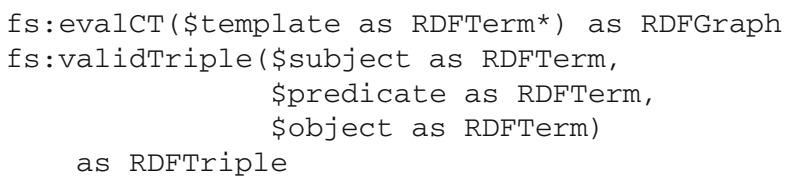

The fs:evalCT function, and hence construct expressions, return elements of the previously defined type RDFGraph, thus allowing the result of construct expressions to be used in a DatasetClause of a subsequent SparqlForClause. In more detail, the $f s: e v a l C T$ function checks the constructed RDF graph for validity according to the conditions described in Definition 1, filtering out any non-valid RDF triples where subjects are literals, predicates are literals or blank nodes, etc. This is illustrated by the following dynamic evaluation rules:

$$
\begin{gathered}
\text { dynEnv } \vdash f_{\text {s:validTriple }}\left(\text { Subj }_{1}, \text { Pred }_{1}, \text { Obj }_{1}\right) \Rightarrow \text { Triple }_{1} \\
\vdots \\
\text { dynEnv } \vdash f \text { s:validTriple }\left(\text { Subj }_{n}, \text { Pred }_{n}, \text { Obj }_{n}\right) \Rightarrow \text { Triple }_{n}
\end{gathered}
$$

The following dynamic evaluation rule for the $f$ s:validTriple function checks, relying on the $f s$ :bnode function defined below, if a triple is valid according to the RDF semantics:

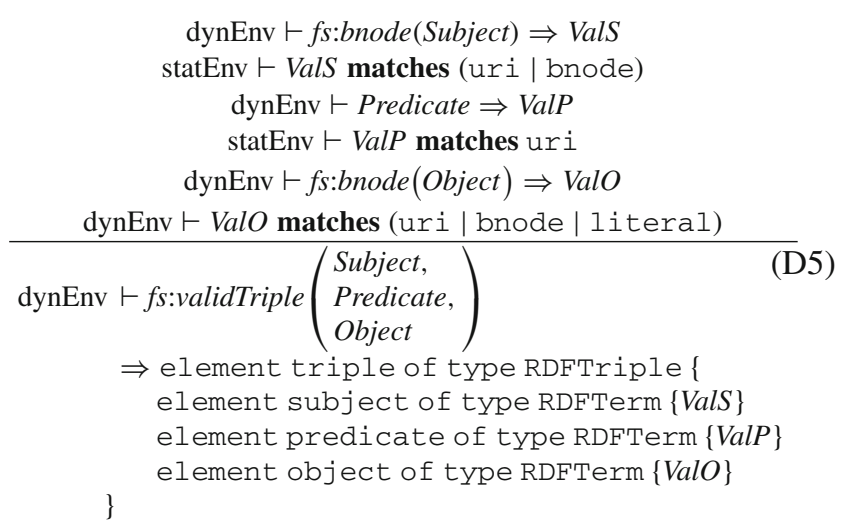

In case any of the subject, predicate or object do not match an allowed type, the empty sequence is returned. Effectively this suppresses any invalid RDF triples from the output graph.

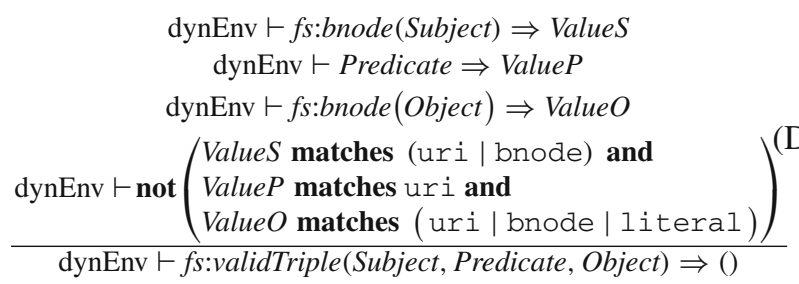

Blank Node Skolemisation In order to comply with the SPARQL construct semantics, all blank nodes inside a ConstructTemplate' need to be skolemised, i.e., for each solution a new distinct blank node identifier needs to be generated. Since we normalise every XQuery for expression and SparqlForClauses by assigning them position variables (as described in Sect. 4.1), we just need to retrieve the available position variables from the static environment component statEnv.posVars, and create the new distinct identifier based on the values of these variables. The $f s$ :bnode function takes care of skolemising blank nodes. If the argument of this function is of type bnode a new blank node identifier is generated using rule (D7):

$$
\begin{aligned}
& \text { dynEnv } \vdash \text { ValueR matches bnode } \\
& \text { statEnv.posVars }=\left(\text { PosVar }_{l}, \ldots, \text { PosVar }_{n}\right) \\
& \text { dynEnv.varValue }\left(\text { PosVar }_{1}\right)=\text { PosValue }_{1} \\
& \text { dynEnv.varValue }\left(\text { Pos Var }_{n}\right)=\text { PosValue }_{n} \\
& \text { dynEnv } \vdash f \text { s:skolemConstant }\left(\begin{array}{l}
\text { Value }, \\
\text { PosValue }_{1}, \\
\ldots, \\
\text { Pos Value }_{n}
\end{array}\right) \Rightarrow \text { ValueRS } \\
& \text { dynEnv } \vdash f s \text { :bnode }(\text { ValueR }) \Rightarrow \\
& \text { element bnode of type xs : string }\{\text { ValueRS }\}
\end{aligned}
$$

Otherwise, $f s$ :bnode returns its argument unchanged as represented by rule (D8):

$$
\frac{\text { dynEnv } \vdash \text { Value matches (uri } \mid \text { Iiteral) }}{\text { dynEnv } \vdash f \text { s:bnode(Value }) \Rightarrow \text { Value }}
$$

Both rules above use the $f$ s:skolemConstant function for the generation of the new identifiers based on the specified blank node label and on positional variables in the dynamic environment. An example of XSPARQL Semantics Evaluation is included in Appendix A.

\subsection{Correspondence Between XSPARQL, XQuery, and SPARQL}

Since XSPARQL syntactically extends XQuery, and-by the remarks in the end of Sect. 4.1-also any SPARQL construct query is syntactically valid in XSPARQL, these queries are considered semantically equivalent to the semantics 
in their base languages. The next propositions formally establish this intuitive correspondence.

The proofs for these propositions and lemmas are included in Appendix C.1-C.3.

Proposition 1 XSPARQL is a conservative extension of XQuery.

A similar correspondence holds for native SPARQL construct queries. We show the equivalence between SPARQL BGP Matching [54, Section 12.3.1] and XSPARQL BGP Matching (presented in Sect. 4.2) and prove the equivalence of XSPARQL semantics for native SPARQL construct queries with those of the SPARQL semantics.

Lemma 1 Given a graph pattern $P$, a dataset $D$ and the $X S P A R Q L$ instance mapping $\mu_{C}$ of the expression context $C$ over which $P$ is evaluated, let $\Omega_{1}=\operatorname{eval}_{x s}\left(D, P, \mu_{C}\right)$ and $\Omega_{2}=\operatorname{eval}(D, P)$ be solution mappings. If vars $(P) \cap$ $\operatorname{dom}\left(\mu_{C}\right)=\emptyset$, then $\Omega_{1}=\Omega_{2} \bowtie\left\{\mu_{C}\right\}$.

We define, based on [54, Section 10.2], the semantics of the SPARQL construct clause to show their equivalence to the XSPARQL construct clause.

Definition 11 (SPARQL construct semantics) Let $C$ be a ConstructTemplate and $\Omega$ a solution sequence. The SPARQL construct returns an RDF graph generated by the set-union of the triples obtained from substituting variables in $C$ with their bindings from $\Omega$ and satisfying the following conditions:

1. any invalid RDF triples that may be produced by the instantiation of the ConstructTemplate are ignored; and

2. blank node labels within the ConstructTemplate are considered scoped to the template for each solution, i.e., if the same label occurs twice in a template, then there will be one blank node created for each solution in $\Omega$, but there will be different blank nodes for triples generated by different query solutions. Blank nodes in the graph template be shared only within the same query solution $\mu_{i} \in \Omega$.

For SPARQL construct queries we can state the following:

Proposition $2 X S P A R Q L$ is a conservative extension of SPARQL construct queries.

\section{Implementation}

In this section we present a prototype implementation of the XSPARQL language. The prototype translates an XSPARQL query into an XQuery query with interleaved calls to a SPARQL engine. The architecture of our implementation is shown in Fig. 14 and consists of three main components:(1) a query

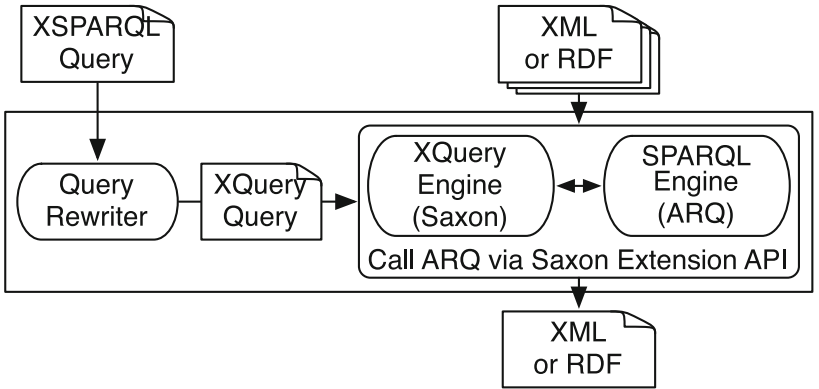

Fig. 14 XSPARQL implementation architecture

rewriter, which turns an XSPARQL query into an XQuery; (2) an XQuery engine for evaluating the XQuery; and (3) a SPARQL engine, for querying RDF from within the rewritten XQuery.

Our current prototype is meant first to demonstrate that XSPARQL can be implemented directly on top of off-theshelf components, and provides convenient means to model and execute XML2RDF/RDF2XML transformations. Second, as illustrated in our evaluation section (Sect. 6) we show that a clever implementation of the XSPARQL language, again integrating an XQuery and a SPARQL engine, but with several optimisations in place, can improve efficiency significantly compared with a naive implementation.

In general it is possible to use any XQuery and SPARQL engines to evaluate XSPARQL queries. The current prototype implements the interface between the XQuery engine, Saxon $9.3,{ }^{11}$ and the SPARQL engine, ARQ 2.8.7, ${ }^{12}$ using the Saxon Extension API which allows calling Java methods from within XQuery queries. The main function, called xsp: sparqlCall, evaluates a SPARQL query and returns its result using the SPARQL XML results format [8]. By using Saxon's extension mechanism the two query engines are tighter integrated allowing a more efficient communication than our former prototype [4] which used a SPARQL endpoint via HTTP to evaluate SPARQL queries. To implement the blank node handling as presented in Sect. 4.2 we changed the behaviour of ARQ accordingly using its Java API. Instead of implementing all newly introduced types as given in Sect. 4.2 as custom types in XQuery, we reuse types as given by the XML Schema of the SPARQL Query Results XML Format, ${ }^{13}$ where the sr:binding type corresponds directly to XSPARQL's RDFTerm type. An RDFGraph, e.g., the result of a ConstructClause, is serialised using Turtle syntax by building the output as xs:string. The remaining types RDFDataset and RDFNamedGraph are adapted accordingly.

\footnotetext{
$11 \mathrm{http} / / /$ saxon.sourceforge.net/.

12 http://jena.sourceforge.net/ARQ/.

13 See http://www.w3.org/2007/SPARQL/result.xsd, for this paper we assume this schema is associated with the namespace prefix $\mathrm{s} r$.
} 
Next we present how SparqlForClauses and ConstructClauses are processed using functions-called rewriting functions - that operate on syntactic objects of XSPARQL and returning an XQuery expression. In the resulting XQuery expressions we assume the namespace prefix $\mathrm{xsp}$ : associated with http://xsparql.deri.org/demo/xquery/xsparql.xquery. This prefix is not allowed to be used in any XSPARQL query and defines XQuery functions (presented below) that are available to the rewriting functions and used as the namespace for any variables introduced by the rewriting, thus avoiding clashes with variables from the XSPARQL query.

SparqlForClause First, our implementation defers SPARQL queries in a SparqlForClause to the external SPARQL engine and extracts the bindings for the SPARQL variables from the SPARQL XML results document that is returned, by the following rewriting function. Let $\mathbb{X S}$ and $\mathbb{X} \mathbb{Q}$ denote the set of all XSPARQL core and XQuery core expressions, respectively. Any SparqlForClauseis translated into an XQuery query that calls the SPARQL engine with a select query according to the rewriting function $\operatorname{tr}: \mathbb{X S} \rightarrow \mathbb{X} \mathbb{Q}$. Given an XSPARQL expression $Q$ of form

for Vars at \$PosVar
DatasetClause
WhereClause
SolutionModifier
return ExprSingle

then $\operatorname{tr}(Q)$ is defined as the XQuery Core expression

$\operatorname{tr}(Q)=$

(1) let \$xsp:results := $\mathrm{xsp}:$ sparqlCall $\left(\begin{array}{l}\text { select Vars DatasetClause } \\ \text { WhereClause } \\ \text { SolutionModifier }\end{array}\right)$ return

(2) for \$xsp:result at \$PosVar in \$xsp:results//sr:result return

(3) $\operatorname{let} \$ v:=\quad$ for each $\$ v \in$ Vars

\$xsp:result/sr:binding[@ @ name =v]/* return

(4) ExprSingle

That is, we implement the $f$ s:sparql formal semantics function by translating $Q$ to a SPARQL select query, which is then executed by the custom runtime function xsp:sparqlCall that receives a SPARQL select query and returns the result in SPARQL's XML result format. The xsp: sparqlCall function also takes care of XSPARQL's BGP matching, as described in Sect. 4.2, by replacing in the SPARQL query any previously bound variables with their current value according to the rules presented in Definition 8, thus mimicking XSPARQL's BGP matching behaviour while relying on an off-the-shelf SPARQL engine. This replacement of variables is performed by producing XQuery code that generates the SPARQL select query string that is given as a parameter to x.sp : sparqlCall function using XQuery's fn:concat function. We parse the query string for variables and, having access to the list of previously declared variables it is possible to determine whether variables should be replaced by their previously bound value or kept as a variable. Within a SparqlForClause, whenever we encounter fresh variables, i.e. that have not been declared before, we leave the variable name as a string within the fn:concat (effectively postponing evaluation of the variable to the SPARQL engine). On the other hand, if a variable has been declared before, the XQuery variable name is inserted into the fn:concat function, meaning that it is evaluated and replaced by its current value when the fn:concat function is evaluated during the execution of the rewritten query. Furthermore, the xsp: sparqlCall function implements the matching blank nodes in nested queries feature (as described in Sect. 4.2): here, we rely on external Java code to call the ARQ API in such a way that blank node labels are preserved over consecutive SPARQL calls that use the same dataset; during query rewriting we trigger the correct matching of blank nodes in nested queries whenever we encounter a SparqlForClause without an explicit DatasetClause) as follows: the respective custom Java code is used to maintain a stack of the previous datasets. More specifically, we collect the blank node identifiers for each dataset created by an explicit DatasetClause in this stack; when a SparqlForClause without explicit DatasetClause is encountered, we take the first element of the stack as the implicit dataset for the SparqlForClause along with its current blank node identifiers.

ConstructClause As for the construction of RDF graphs (i.e., whenever the ReturnClause is a ConstructClause), our implementation within XQuery simply produces a string in Turtle syntax, where we need to ensure that each produced RDF triple is syntactically valid. This is implemented by means of a number of additional custom functions. First, the auxiliary function xsp:rafterm(\$VarName), presented in Fig. 19a (Appendix B), returns the correctly formatted RDF term corresponding to the variable's value in Turtle syntax given a variable of a SPARQL result type. This is done by matching the type of the variable and adding the necessary syntactic elements for each type. Next, the xsp:validTriple presented in Fig. 19b (Appendix B) implements the semantics function $f$ s:validTriple by calling the xsp:rdfTerm function to correctly format triples to text (using the Turtle syntax); xsp:validTriple further uses the auxiliary functions xsp:validsubject, xsp:validPredicate and xsp:validobject that, respectively, determine, according to the RDF semantics, if their argument is a valid subject, predicate or object. Also available to our implementation are the functions xsp:isBlank, xsp:isURI and Xsp:istiteral which determine, respectively, if a term is a blank node, URI or literal. Our implementation of the $f$ s:skolemConstant function consists of appending all 
the position variables from the static context (that are stored in the statEnv.pos Vars component) to the respective blank node identifier using " _" as a separator, represented here by the rewriting function

$$
\begin{aligned}
& \operatorname{tr}_{s k}\left(\$ B \text { NodeName, }\left\{\$ \text { PosVar }_{1}, \cdots, \$ \text { PosVar }_{n}\right\}\right)= \\
& \text { fn:concat ("_:", \$BNodeName, "-", \$PosVar }, 1, \cdots,) \text {. }
\end{aligned}
$$

Finally, the function xsp : evalCT (without details) implements $f$ :evalCT by simply concatenating all the triples generated by the xsp:validTriple function to a string representation of the RDF graph to be constructed in Turtle syntax.

The next lemma states that the results of the evaluation of a Basic Graph Pattern $P$ under XSPARQL BGP matching semantics can be determined based on the results of evaluating $\mu(P)$ under SPARQL semantics. The proofs for the following proposition and lemma are included in Appendices C.4 and C.5.

Lemma 2 Let $P$ be a BGP, $D$ a dataset, and $\mu$ the XSPARQL instance mapping of $P$. Considering $P^{\prime}=\mu(P)$, we have that eval $x s(D, P, \mu)=\operatorname{eval}\left(D, P^{\prime}\right) \bowtie\{\mu\}$.

The following result presents the equivalence of our implementation function $t r$ and the XSPARQL semantics. ${ }^{14}$

Proposition 3 Let $Q$ be a SparqlForClauseofform (Q1) and dynEnv the dynamic environment of $Q$, then dynEnv $\vdash Q \Rightarrow$ Val if and only if dynEnv $\vdash \operatorname{tr}(Q) \Rightarrow$ Val.

\section{Towards Optimisations of Nested for Expressions}

In this section we present different rewriting strategies for XSPARQL queries containing nested expressions. We are specifically interested in nested expressions with an inner SparqlForClause, as the number of interleaved calls to the SPARQL engine can be reduced drastically using these rewritings. As the cost inherent with XQuery for clauses is much lower, we do not present different rewritings for nested expressions where the inner expression is an XQuery for. These different rewritings proposed in this section constitute the initial step towards defining a set of optimisations for the current implementation of the XSPARQL language.

We start by presenting the definitions and conditions under which we can perform these rewritings.

Definition 12 (Dependent Join) We call two nested XSPARQL for expressions (ForClause or SparqlForClause), where the inner expression is a SparqlForClause and at least one variable in the inner expression is bound by the outer expression, a dependent join. The shared variables between the for expressions are called dependent variables.

\footnotetext{
$\overline{14}$ Please note that, for presentation purposes, we are omitting the initial empty line in case the proof trees require no premises.
}

Note that the strategies presented here are only applicable for dependent joins satisfying the following restrictions:

1. An explicit DatasetClause of the inner query needs to be statically determined i.e., it cannot be determined based on variables bound from the outer expression;

2. The return clause of the inner expression can not be a ConstructClause; and

3. The dependent variable in the inner query's graph pattern must be strictly bounded as defined next.

Definition 13 (Strict Boundedness) The set of strictly bound variables in a graph pattern $P$, denoted $b \operatorname{Vars}(P)$, is recursively defined as follows: if $P$ is

- a basic graph pattern, then $b \operatorname{Vars}(P)=\operatorname{vars}(P)$;

- $\left(P_{1} P_{2}\right)$, then $b \operatorname{Vars}(P)=b \operatorname{Vars}\left(P_{1}\right) \cup \operatorname{bVars}\left(P_{2}\right)$;

- $\left(P_{1}\right.$ optional $\left.P_{2}\right)$, then $b \operatorname{Vars}(P)=b \operatorname{Vars}\left(P_{1}\right)$;

- $\left(P_{1}\right.$ union $\left.P_{2}\right)$, then $b \operatorname{Vars}(P)=b \operatorname{Vars}\left(P_{1}\right) \cap b \operatorname{Vars}\left(P_{2}\right)$;

- $\left(\operatorname{graph} i P_{1}\right)$, then $b \operatorname{Vars}(P)=b \operatorname{Vars}\left(P_{1}\right) \cup(\{i\} \cap \mathbb{V})$; and

- $\left(P_{1}\right.$ filter $\left.R\right)$, then $b \operatorname{Vars}(P)=b \operatorname{Vars}\left(P_{1}\right)$.

Informally, the dependent variables must occur (i) in a basic graph pattern, (ii) in every alternative of unions pattern, and (iii) also outside of the optional graph pattern in case of optionals. Strict boundedness essentially ensures that the join variable does not occur only in a filter expression, which would lead to problems in case the inner expression is called unconstrained, see below.

The rewritings introduced for the implementation of dependent joins can be grouped into two categories, depending whether the join is performed in XQuery or SPARQL. For performing the join in XQuery, we use already known join algorithms from relational databases, namely nestedloop joins or sort-merge joins. For performing the join in SPARQL, if the outer expression is a SparqlForClause we can implement the join by rewriting both the inner and the outer expressions into a single SPARQL call. In case the outer query consists of an XQuery ForClause, we can still consider this approach, but we need to convert the result of the outer XQuery ForClause to an RDF graph, for instance relying on a SPARQL engine that supports SPARQL Update [32] to add this temporary graph to a triple store. The proofs for the propositions presented in this section are included in Appendices C.6-C.8.

\subsection{Dependent Join implementation in XQuery}

The intuitive idea with these rewritings is, instead of using the naïve rewriting that performs one SPARQL query for each iteration of the outer expression, to execute only one unconstrained SPARQL query, before the outer query. The resulting set of SPARQL solution mappings is then joined in 
XQuery with the results of the outer expression, using one of the following strategies.

The straightforward way to implement the join over dependent variables directly in XQuery is by nesting two XQuery for expressions, much like a regular nested-loop join [1] in standard relational databases. In our proposed rewriting, the join consists of restricting the values of variables from the inner expression to the values taken from the current iteration of the outer expression, which does not require an incremental solution construction step. The approach for query rewriting applied to XSPARQL is similar to already known optimisations from the relational databases realm and also presented for XQuery queries by [47].

Similarly to Sect. 5, we will describe the implementation of this nested-loop join by means of the rewriting function $o p t_{n l}$. We use $A \triangle B=(A \cup B) \backslash(A \cap B)$ to denote the symmetric difference of two sets $A$ and $B$.

Let $Q$ be an XSPARQL expression of form

(1) for $\$$ Var $^{\text {out }}$ at $\$$ PosVar $^{\text {out }}$ in ExprSingle 1 return

(2) for Vars $^{\text {in }}$ at $\$$ PosVar ${ }^{\text {in }}$

DatasetClause WhereClause SolutionModifier

(3) return ExprSingle 2

the application of the rewriting function $\operatorname{opt}_{n l}(Q)$ can be split into two cases:

- if ExprSingle ${ }_{1}$ and ExprSingle $e_{2}$ do not contain any occurrences of $(\mathrm{Q} 2)$ then, assuming $\operatorname{Vars}^{s p}=\operatorname{Vars}($ WhereClause), we have that

$o p t_{n l}(Q)=$

(1) let \$xsp:results := xsp: sparqlCall $\left(\begin{array}{l}\text { select }\left\{\$ \text { Var }^{\text {out }}\right\} \cup \text { Vars }^{\text {in }} \\ \text { DatasetClause } \\ \text { WhereClause } \\ \text { SolutionModifier }\end{array}\right)$

return

(2) for $\$ \mathrm{Var}^{\text {out }}$ at $\$$ PosVar $^{\text {out }}$ in ExprSingle 1 return

(3) for \$xsp:result at $\$$ PosVar $^{\text {in }}$

in \$xsp:results//sr:result return

(4)

if $\left(\right.$ join $\left._{n l}\left(\begin{array}{l}\left\{\begin{array}{l}\left.\$ \text { Var }^{\text {out }}\right\} \cap \text { Vars }^{s p}, \\ \text { xsp:result }\end{array}\right.\end{array}\right)\right)$ then

(5)

let $\$ v:=$

for each $\$ v \in\left\{\right.$ Var $\left.^{\text {out }}\right\} \triangle$ Vars $^{\text {sp }}$

\$xsp:result/sr:binding[@ $@$ name=v]/* return

(6) ExprSingle

(7) else ()

Note that here we slightly abuse notation, using ' $U$ ' to denote the concatenation of two lists of variables. The auxiliary function join $_{n l}$ aggregates the actual joincomparison in an XPath expression, where two variables are considered compatible if the outer value is a blank node, their values are equal, or the inner value ( $\$$ VarRes $_{i}$ ) is unbound:

$$
\begin{aligned}
& \text { join }_{n l}\left(\left\{\text { Var }_{l}, \cdots, \$ \operatorname{Var}_{n}\right\}, \$ \text { res }\right)= \\
& \left(\begin{array}{l}
\text { xsp: isBlank }\left(\$ \operatorname{Var}_{l}\right) \text { or } \\
\text { fn:empty }\left(\$ r e s / \text { sr:binding }\left[@ \text { name }=\operatorname{Var}_{1}\right] / *\right) \text { or } \\
\left(\$ \operatorname{Var}_{1} \text { eq } \$ \text { res } / \text { sr:binding[ }\left[\text { name }=\operatorname{Var}_{1}\right] / *\right)
\end{array}\right) \\
& \text { and... } \\
& \text { and }\left(\begin{array}{l}
\text { xsp:isBlank }\left(\$ \operatorname{Var}_{n}\right) \text { or } \\
\text { fn:empty }\left(\$ r e s / \text { sr:binding }\left[@ \text { name }=\operatorname{Var}_{n}\right] / *\right) \\
\text { or } \\
\left(\$ \operatorname{Var}_{n} \text { eq } \$ \text { res } / \text { sr:binding }\left[@ \text { name }=\operatorname{Var}_{n}\right] / *\right)
\end{array}\right)
\end{aligned}
$$

- otherwise:

$$
\begin{aligned}
& \text { opt }_{n l}(Q)= \\
& \text { opt }_{n l}\left(\begin{array}{c}
\text { for } \$ \text { Var }^{\text {out }} \text { at } \$ \text { PosVar }^{\text {out }} \text { in } \text { opt }_{n l}\left(\text { ExprSingle }_{1}\right) \\
\text { return } \\
\text { for Vars }{ }^{\text {in }} \text { at } \$ \text { PosVar }^{\text {in }} \\
\text { DatasetClause WhereClause SolutionModifier } \\
\text { return } \text { opt }_{n l}\left(\text { ExprSingle }_{2}\right)
\end{array}\right) \\
& \text { When } Q \text { is an XSPARQL expression of form }
\end{aligned}
$$

the application of the rewriting function $\operatorname{opt}_{n l}(Q)$ can be split into two cases:

- in case ExprSingle does not contain any occurrences of (Q3) then, considering $\operatorname{Vars}^{s p}=\operatorname{vars}\left(\right.$ WhereClause $^{\text {in }}$ ) being the set of variables from the inner WhereClause, we have that

opt $_{n l}(Q)=$

(1) let \$xsp:res_in :=

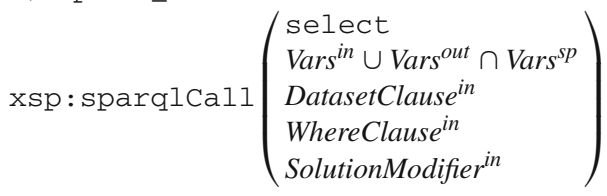

return

(2) let $\$ x$ sp:res_out := xsp : sparqlCall $\left(\begin{array}{l}\text { select Varsout } \\ \text { DatasetClause } \\ \text { WhereClause }^{\text {out }} \\ \text { SolutionModifier }\end{array}\right)$

return

(3) for \$xsp:rout at \$PosVar out

in \$xsp:res_out//sr:result return

(4)

let $\$ v:=$ for each $\$ v \in \operatorname{Vars}^{\text {out }}$

\$xsp:rout/sr:binding[@ name $=v] / *$ return

(5) for \$xsp:rin at \$PosVar ${ }^{\text {out }}$

in \$xsp:res_in//sr:result return

(6) if $\left(\operatorname{join}_{s r}\left(\begin{array}{l}\text { Vars }^{\text {out }} \cap \text { Vars }^{\text {sp }}, \\ \$ \mathrm{xsp}: r e s \_o u t, \\ \$ \mathrm{xsp}: r e s \_i n\end{array}\right)\right)$ then

(7) $\quad$ let $\$ v:=\quad$ for each $\$ v \in$ Vars $^{\text {out }} \triangle \operatorname{Vars}^{s p}$

\$x.sp:res_in/sr:binding[@ name =v]/* return

(8) ExprSingle

(9) else () 


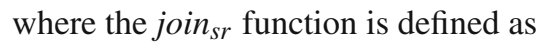

$$
\begin{aligned}
& \text { join }_{s r}\left(\left\{\$ \operatorname{Var}_{1}, \cdots, \$ \operatorname{Var}_{n}\right\}, \text { \$resOut, } \$ \text { resIn }\right)= \\
& \text { join } \left._{n l}\left(\left\{\text { resOut/sr:binding[ } @ \text { name }=\operatorname{Var}_{1}\right] / *\right\}, \$ \text { resIn }\right) \\
& \text { and } \cdots \text { and } \\
& \text { join }_{n l}\left(\left\{\text { \$resOut/sr:binding }\left[@ \text { name }=\operatorname{Var}_{n}\right] / *\right\}, \$ \text { resIn }\right) \text {. }
\end{aligned}
$$

- otherwise:

$$
\begin{aligned}
& { }_{o p t} t_{n l}(Q)=
\end{aligned}
$$

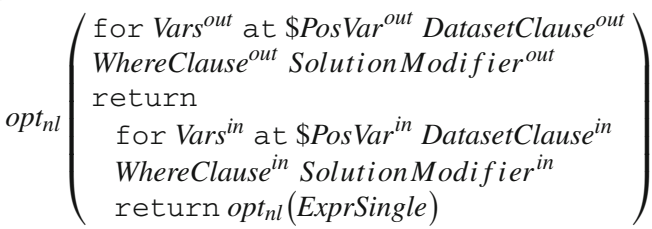

This rewriting to the nested-loop join reduces the number of needed SPARQL calls from $1+N$ (where $N$ is the number of iterations of the outer expression) to two SPARQL calls.

Next we show that the $o p t_{n l}$ rewriting function is sound and complete.

Proposition 4 Let $Q$ be a XSPARQL expression of form $(\mathrm{Q} 2)$ or $(\mathrm{Q} 3)$ and dynEnv the dynamic environment of $Q$, then dynEnv $\vdash Q \Rightarrow$ Val if and only if dynEnv $\vdash$ opt $_{n l}(Q) \Rightarrow$ Val.

\subsection{Dependent Join Implementation in SPARQL}

This form of rewriting of nested expressions aims at improving the runtime of the query by delegating the execution of the join to the SPARQL engine, as opposed to performing the join within XQuery only.

SparqlForClause within a SparqlForClause. For nested expressions where both expressions consist of SparqlForClauses we can implement the join by rewriting the SparqlForClauses into a single SPARQL query. The idea here is that a join encoded as nested SparqlForClauses in XSPARQL can just be implemented by a SPARQL query that merges the where clauses of the outer and inner SparqlForClause. However, there are some restrictions to the applicability of this rewriting: (i) both queries must be done over the same dataset; (ii) apart from order by, no other solution modifiers can be used in the queries; and (iii) the original queries must not require any nesting of the XML output or use of aggregators.

As indicated before, for the next rewriting we are only allowing the order by solution modifier and the concatenation of "order by $\$ 01$ " and "order by $\$ 02$ " is "order by \$o1 \$o2". For presentation purposes, GGP and $O C$ are, respectively, a short representation for GroupGraphPattern and OrderCondition.
For an XSPARQL query $Q$ of form

(1) for Vars ${ }^{\text {out }}$ at \$PosVar ${ }^{\text {out }}$ DatasetClause

(2) where GGP ${ }^{\text {out }}$

(3) order by $O C^{\text {out }}$

(4) return

(5) for Vars $^{\text {in }}$ at $\$$ Pos Var $^{\text {in }}$ DatasetClause

(6) where $G G P^{\text {in }}$

(7) order by $O C^{\text {in }}$

(8) return ExprSingle

then

- in case ExprSingle does not contain any occurrences of (Q4), we have that

$o p t_{s r}(Q)=$

(1) let \$xsp:results :=

$$
\text { xsp: sparqlCall }\left(\begin{array}{l}
\text { select Vars }{ }^{\text {out }} \cup \text { Vars }^{\text {in }} \\
\text { DatasetClause } \\
\text { where }\left\{G G P^{\text {out }} . G G P^{\text {in }}\right\} \\
\text { order by } O C^{\text {out }} O C^{\text {in }}
\end{array}\right)
$$

return

(2) for \$xsp:result at \$PosVar out

in \$xsp:results//sr:result return

(3) $\quad$ let $\$ v:=$

for each $\$ v \in$ Vars

\$xsp:result/sr:binding[@ $@$ ame $=\$ v] / *$ return

(4) ExprSingle

Please note that the group graph patterns $G G P_{1}$ and $G G P_{2}$ include the surrounding curly braces: $\{$ and $\}$.

- otherwise,

$$
\begin{aligned}
& \text { opt }_{s r}(Q)= \\
& \text { opt }_{s r}\left(\begin{array}{l}
\text { for Vars }{ }^{\text {out }} \text { at } \$ \text { PosVar } \\
\text { where } G G P^{\text {out }} \\
\text { order by } \text { OC }^{\text {out }} \\
\text { return } \\
\text { for Vars }{ }^{\text {in }} \text { at } \$ \text { PosVar }^{\text {in }} \text { DatasetClause } \\
\text { where GGP } \\
\text { order by } \text { OC }^{\text {in }} \\
\text { return opt } \\
\text { sr }(\text { ExprSingle })
\end{array}\right)
\end{aligned}
$$

Proposition 5 Let $Q$ an XSPARQL expression of form (Q4) and dynEnv the dynamic environment of $Q$; then dynEnv $\vdash$ $Q \Rightarrow$ Val if and only if dynEnv $\vdash$ opt $_{s r}(Q) \Rightarrow \mathrm{Val}$.

SparqlForClause within an XQuery for In case the outer expression is an XQuery for a similar strategy of deferring the join to a single SPARQL query is still possible. Since the optimisation proposed here does not preserve the ordering of results, it can only be applied if the order of the outer XQuery expression is not relevant. Cases where ordering can be disregarded in XQuery, as discussed by [35], include not only the unordered ordering mode in XQuery but also the use of aggregate functions and other built-in functions or the quantifiers some and every. This optimisation relies on first transforming the outer expressions' XML results into $\mathrm{RDF}$ and then joining this newly created RDF graph with the inner SparqlForClause's where pattern in a single SPARQL 
query. To implement this, we can, for instance, rely on a triple store with support for named graphs to temporarily store the RDF data corresponding to the outer XQuery for expression's bindings for dependent variables. We can then execute a combined query with an adapted graph pattern that joins the pattern in the where clause of the inner SparqlForClause with the bindings stored in the newly created named graph. The opt $_{n g}$ rewriting function (presented below) starts by creating RDF triples representing the XML input which are then collected into the variable \$xsp:ds that corresponds to the RDF graph to be inserted into the triple store. This operation is achieved by the XSPARQL functions xsp: createNG that returns a URI for the newly inserted RDF named graph, which is distinct from any other URIs for named graphs used in the query, while finally the function xsp: deleteNG takes care of deleting the temporary graph. Let $Q$ be an XSPARQL expression of form

(1) for \$VarName OptTypeDeclaration

(2) OptPositionalVar in ExprSingle

(3) return for Vars DatasetClause WhereClause

(4) SolutionModifier return ExprSingle 2

then,

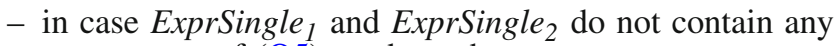
occurrences of (Q5), we have that

$\operatorname{opt}_{n g}(Q)=$

(1) let $\$ \mathrm{xsp}: \mathrm{ds}:=$

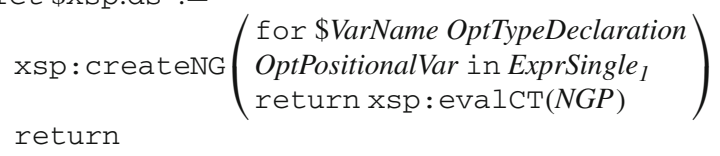

(2) let $\$ x$ sp:results := x.sp: sparqlCal1 $\left(\begin{array}{l}\text { select Vars } \cup\{\text { \$VarName }\} \\ \text { DatasetClause } \cup \\ \quad\{\text { from named \$xsp:ds }\} \\ \text { WhereClause } \cup \\ \quad \text { where }\{\text { graph \$xsp:ds } \\ \text { NGP }\} \\ \text { SolutionModifier }\end{array}\right)$

return

(3) for \$xsp:result at \$xsp:result_pos in \$xsp:results//sr:result return

(4) let $\$ v:=$ for each $\$ v \in$ Vars $\cup\{\$$ VarName $\}$

\$xsp:result/sr:binding[@ name =\$v]/*

(5) return (ExprSingle $2, \mathrm{xsp}: \operatorname{deleteNG}(\$ \mathrm{xsp}: \mathrm{ds}))$

where NGP is the graph pattern $\{[]$ :value $\$$ VarName $\}$. - otherwise,

$$
\begin{aligned}
& \text { opt }_{n g}(Q)= \\
& \text { opt }_{n g}\left(\begin{array}{l}
\text { for } \$ \text { VarName OptTypeDeclaration } \\
\text { OptPositionalVar in opt } \text { nd }_{n g}\left(\text { ExprSingle }_{1}\right) \\
\text { return for Vars DatasetClause WhereClause } \\
\text { SolutionModifier return } \text { opt }_{n g}\left(\text { ExprSingle }_{2}\right)
\end{array}\right)
\end{aligned}
$$

Proposition 6 Let $Q$ be an XSPARQL expression of form (Q5) and dynEnv the dynamic environment of $Q$, then dynEnv $\vdash Q \Rightarrow$ Val if and only if dynEnv $\vdash$ opt $_{n g}(Q) \Rightarrow$ Val.

\section{Experimental Evaluation}

In this section we present an experimental evaluation of our prototype presented in Sect. 5 using a novel benchmark suite, called XMarkRDF, that is based on the well-known XMark benchmark suite for XQuery. We compare our XSPARQL prototype with the SPARQL2XQuery engine, an implementation of the direct translation of SPARQL to XQuery presented by [33] and test-where possible - the effects of the optimisations presented in Sect. 6.

A detailed description of the XMarkRDF benchmark suite is included in Appendix D. We denote the 20 original XMark queries as $q_{1}-q_{20}$ and the variants of the nested queries to which we apply our different rewritings as $q_{8}^{\prime}-q_{11}^{\prime}$ and $q_{8}^{\prime \prime}-$ $q_{11}^{\prime \prime}$. Further details regarding these queries are included in Appendix D.

We would like point the reader to available benchmark results for XQuery ${ }^{15}$ and SPARQL ${ }^{16}$ which present better results than our XSPARQL implementation benchmarked in this section. However, the comparison with such native SPARQL and XQuery engines is beyond scope of the paper since we specifically address a combined use case, where components from both XQuery and SPARQL are needed. The benchmark queries presented here cannot be comparably solved by relying on a single SPARQL or XQuery engine.

\subsection{Experimental Setup}

Using the data generators and translators, provided by the XMark benchmark and the XSPARQL translation to RDF (as presented in Sect. 6), we created datasets with scaling factors of $0.01,0.02,0.05,0.1,0.2,0.5$, and 1.0 and translated them into XMarkRDF. An overview of the generated data is presented in Appendix D, including dataset sizes and, for each of the dataset size considered, the number of persons and item categories modelled.

Furthermore, we converted the XMarkRDF datasets into the RDF/XML format required by the SPARQL2XQuery system. The resulting dataset sizes and translation times for

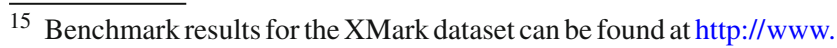
monetdb.org/XQuery/Benchmark/XMark/ and http://www.informatik. uni-freiburg.de/ mschmidt/smp/xmark.html, retrieved 20-04-2012.

16 Benchmark evaluation of RDF stores can be found at http:// www4.wiwiss.fu-berlin.de/bizer/BerlinSPARQLBenchmark/results/ V6/index.html, retrieved 20-04-2012.
} 
Table 1 Query response times (in seconds) of the 2MB dataset. Query rewriting error (err)

\begin{tabular}{lllllllllll}
\hline & $q_{1}$ & $q_{2}$ & $q_{3}$ & $q_{4}$ & $q_{5}$ & $q_{6}$ & $q_{7}$ & $q_{8}$ & $q_{9}$ & $q_{10}$ \\
\hline$X S$ & 9.25 & $\mathbf{1 0 . 6 5}$ & $\mathbf{1 0 . 4 3}$ & 9.43 & 10.15 & 11.38 & $\mathbf{1 1 . 9 7}$ & 358.27 & 355.71 & $\mathbf{3 5 . 8 9}$ \\
$S 2 X Q$ & $\mathbf{2 . 6 3}$ & 19.47 & err & $\mathbf{3 . 7 1}$ & $\mathbf{2 . 8 2}$ & $\mathbf{2 . 5 8}$ & err & $\mathbf{3 . 4 4}$ & $\mathbf{1 8 . 9 1}$ & 178.71 \\
\hline & $q_{11}$ & $q_{12}$ & $q_{13}$ & $q_{14}$ & $q_{15}$ & $q_{16}$ & $q_{17}$ & $q_{18}$ & $q_{19}$ & $q_{20}$ \\
\hline$X S$ & 371.46 & $\mathbf{8 1 . 9 6}$ & 10.83 & 10.04 & 11.61 & 11.66 & 10.14 & 10.93 & 10.73 & 19.93 \\
$S 2 X Q$ & $\mathbf{1 7 . 9 9}$ & 128.89 & $\mathbf{3 . 9 3}$ & $\mathbf{2 . 7 2}$ & $\mathbf{3 . 0 0}$ & $\mathbf{3 . 1 2}$ & $\mathbf{7 . 5 8}$ & $\mathbf{1 0 . 9 2}$ & $\mathbf{3 . 0 5}$ & $\mathbf{1 6 . 6 4}$ \\
\hline
\end{tabular}

the different scaling factors of the XMarkRDF dataset are also presented in Appendix D.

The benchmark system consists of a dual-core AMD Opteron $2502.4 \mathrm{GHz}, 4 \mathrm{~GB}$ memory running a 64-bit installation of Ubuntu 10.04.1 LTS. For the XQuery engine, we rely on Saxon version 9.3 Enterprise Edition and Java version 1.6.0 64 bit. For evaluating SPARQL queries we used ARQ 2.8.7. We ran each query with a timeout of $10 \mathrm{~min}$ per query, with the Java Heap size set to 1GB and the Saxon configuration set as schema-unaware. The response time of each query was measured using GNU time 1.7 and the process startup time was deduced to each response time. For the evaluation we defined the following run configurations:

XS using the XSPARQL implementation over the XMarkRDF datasets (translated data and queries) without optimisation;

$\mathbf{X S}_{Z}$ using the XSPARQL implementation over the XMarkRDF datasets (translated data and queries) with nested expression optimisation $o p t_{Z}$ for $Z \in\{n l, s r, n g\}$;

S2XQ using the SPARQL2XQuery implementation over the translation of the XMarkRDF datasets into the required XML format $\left(\mathrm{XMarkRDF}_{S 2 \mathrm{XQ}}\right)$ without optimisation; and

$\mathbf{S 2 X Q _ { Z }}$ using the SPARQL2XQuery implementation over the translation of the XMarkRDF datasets into the required XML format $\left(\mathrm{XMarkRDF}_{S 2 X Q}\right)$ with nested expression optimisation $\mathrm{opt}_{Z}$ for $Z \in\{n l, s r\}$.

\subsection{Results and Interpretation}

The response times of the $X S$ and $S 2 X Q$ runs for the benchmark queries over the $2 \mathrm{MB}$ dataset size are shown in Table 1. We present the 2MB dataset as it is the largest dataset our unoptimised implementation can process within the time limit of $10 \mathrm{~min}$. Both the data and query translation times are not measured in our benchmarks since this process can be done a priori. The response times for the XMark queries evaluated using the Saxon XQuery engine are not presented in this table since these queries do not cater for our heterogenous data sources scenario.
The comparison of the response times of the different rewriting functions presented in Sect. 6 is shown graphically in Figs. 15 and 16. The response times of these queries for the $2 \mathrm{MB}$ are presented in Table 2 as a reference, where $n / a$ indicates that the combination of query and optimisation is not applicable.

We next present the interpretation of the benchmark results when comparing to the SPARQL2XQuery system and then proceed to then interpretation of the results from the different rewriting strategies.

Evaluation of $X S$ and $S 2 X Q$ without optimisation Table 1 shows that for most of the queries the $S 2 X Q$ runs are faster than the interleaved calls to a SPARQL engine in the $X S$ runs. Even considering that the response times do not include the data translation times, this suggests that an alternative implementation of XSPARQL where the SPARQL queries are translated into native XQuery is a viable alternative to interleaving calls to a SPARQL engine. However, for such translations to be possible we need access to the full RDF dataset to perform the query translation which is not possible for example in the case where we are querying data behind a SPARQL endpoint. Another issue related to the implementation of the SPARQL2XQuery system is that response times deteriorate considerably for larger datasets. This was observed for all the queries in the benchmark and can be seen in the graphs of Figs. 15 and 16.

Queries $q_{8}-q_{12}$ have the highest execution times of all the benchmark queries since they contain nested expressions (as can be seen in $q_{9}$ presented in Fig. 20). For these nested queries, our interleaved XSPARQL implementation can only handle small datasets: the $2 \mathrm{MB}$ dataset is the largest for which all queries finish within the time limit and for the 20MB dataset all queries result in a timeout. For these nested queries we applied the different optimisations described in Sect. 6 and next we present their benchmark evaluation.

Evaluation of $X S$ and $S 2 X Q$ with Nested Expression Optimisation. As we can see from Table 2 and Figs. 15 and 16, the $o p t_{n l}$ optimisation provides significant reduction in the query evaluation times. For queries $q_{8}, q_{9}$, and $q_{11}$ the difference in response times is one order of magnitude. 


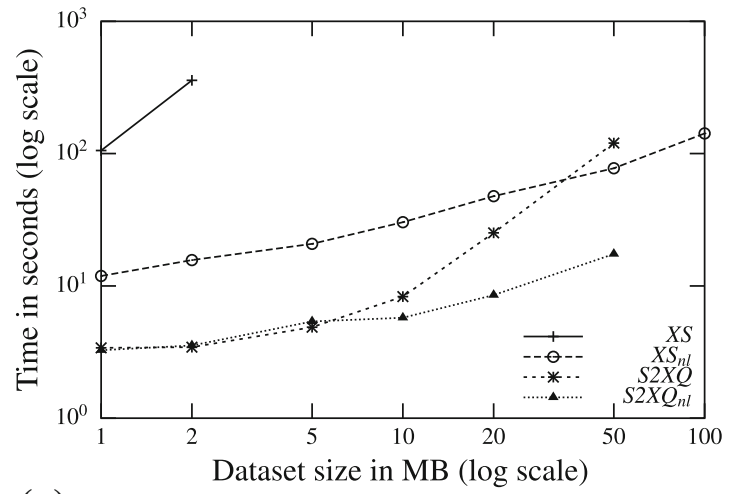

(a) Query $q_{8}$

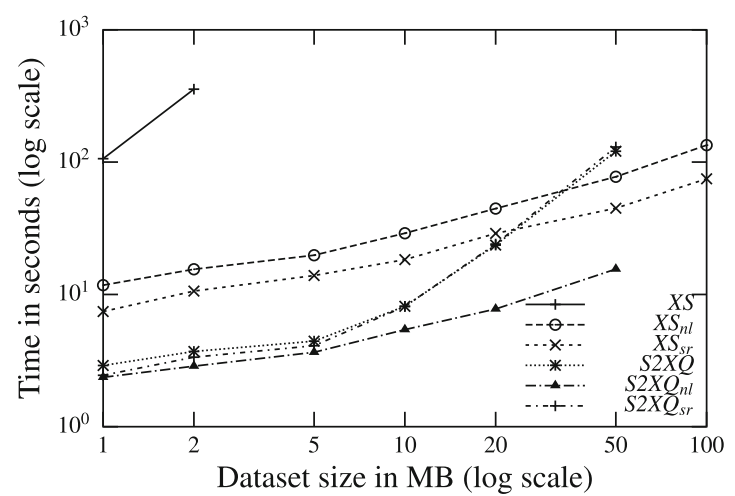

(c) Query $q_{8}^{\prime}$

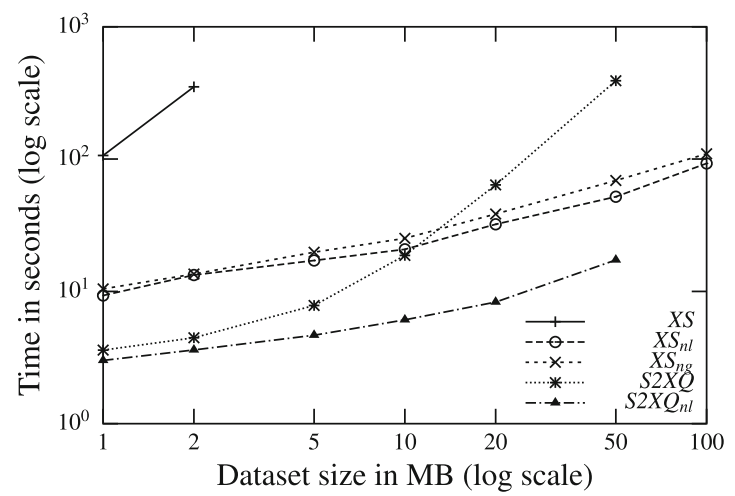

(e) Query $q_{8}^{\prime \prime}$

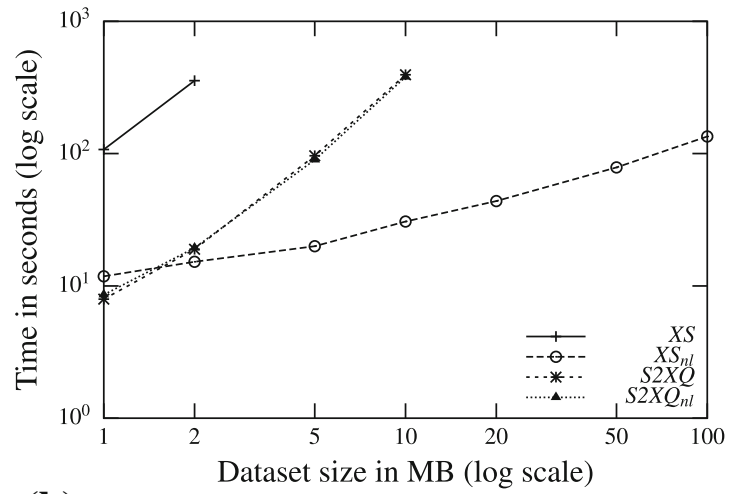

(b) Query $q_{9}$

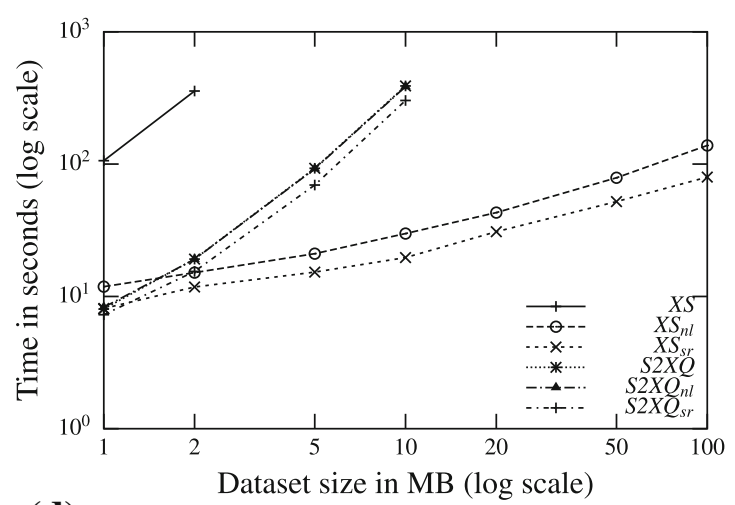

(d) Query $q_{9}^{\prime}$

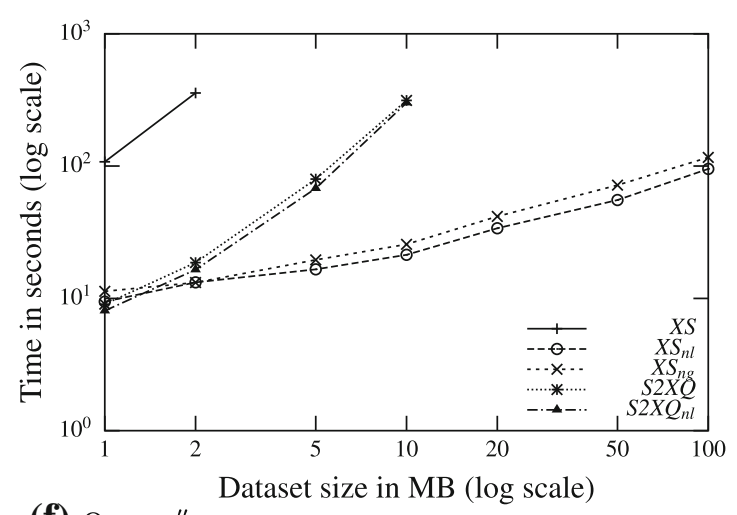

(f) Query $q_{9}^{\prime \prime}$

Fig. 15 Query response times for (variants of) $q_{8}$ and $q_{9}$ on all XMarkRDF datasets

The improvement in the execution time for query $q_{10}$ is less drastic. This can be explained by the fact that the outer expression of $q_{10}$ iterates over "categories" which increases at a much smaller rate than "persons" do in the outer expressions of queries $q_{8}, q_{9}$, and $q_{11}$ (cf. Appendix D).

However, for the $S 2 X Q$ runs this optimisation provides virtually no improvement in the query response times for queries $q_{8}$ and $q_{9}$ and their variants. In queries $q_{10}, q_{11}$, $q_{10}^{\prime}$, and $q_{11}^{\prime}$ we can observe an improvement in response times. This can be attributed to the fact that the rewriting for queries $q_{10}$ and $q_{11}$ and their variants are not as suitable for optimisation by the XQuery engine when compared with queries $q_{8}$ and $q_{9}$. For these cases our rewriting strategy is capable of performing the optimisation task for the XQuery engine.

For the $X S$ run, it is possible to see in Fig. 15c, d that opt $t_{s r}$ (presented in Sect. 6.2) is generally more efficient in terms of response times than the XQuery based. This can be justified by the smaller amount of information that is necessary to transfer from SPARQL to the XQuery engine. This effec- 


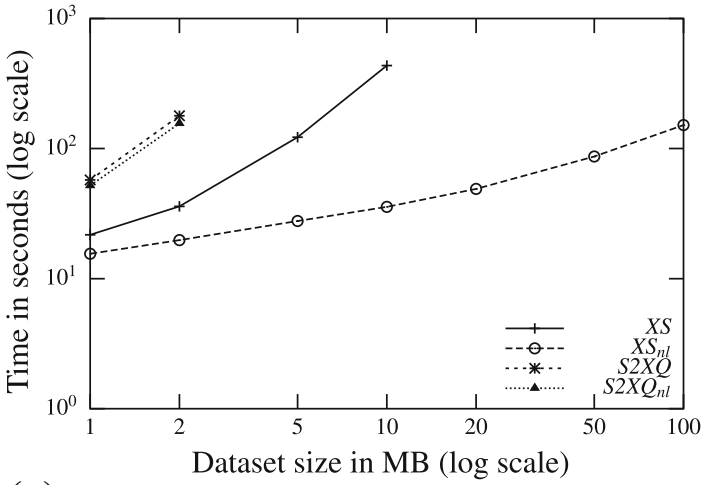

(a) Query $q_{10}$

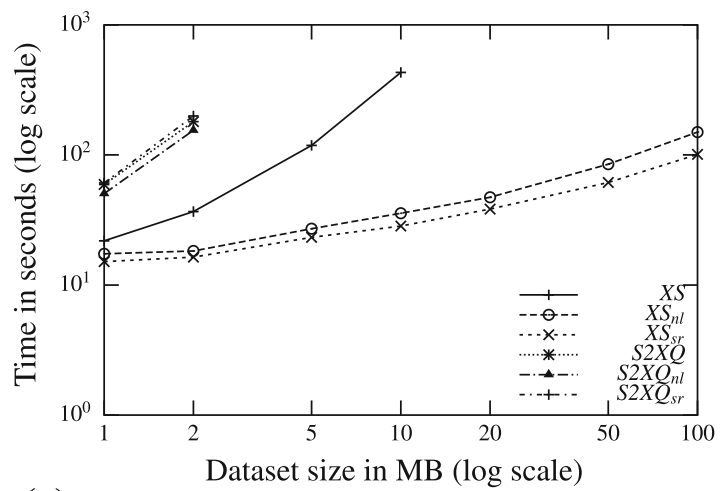

(c) Query $q_{10}^{\prime}$

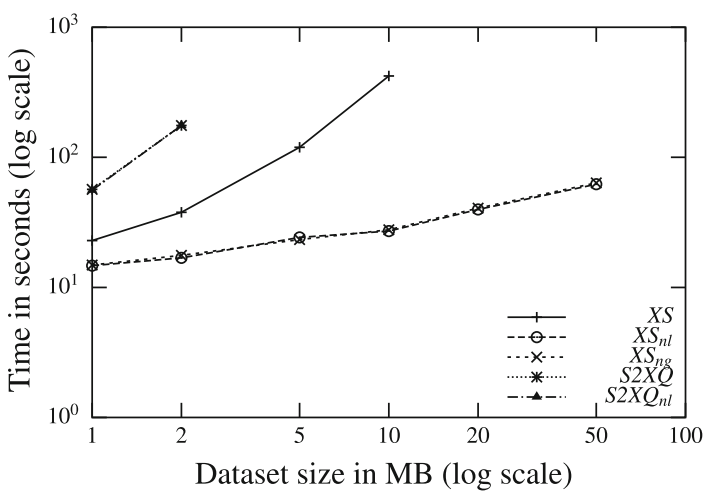

(e) Query $q_{10}^{\prime \prime}$

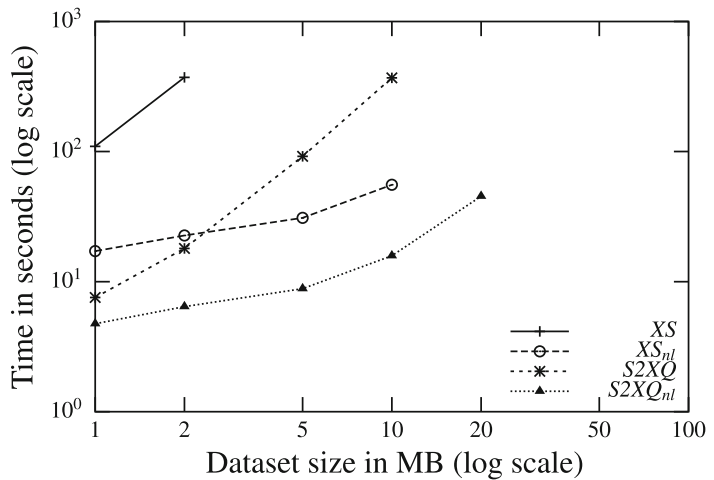

(b) Query $q_{11}$

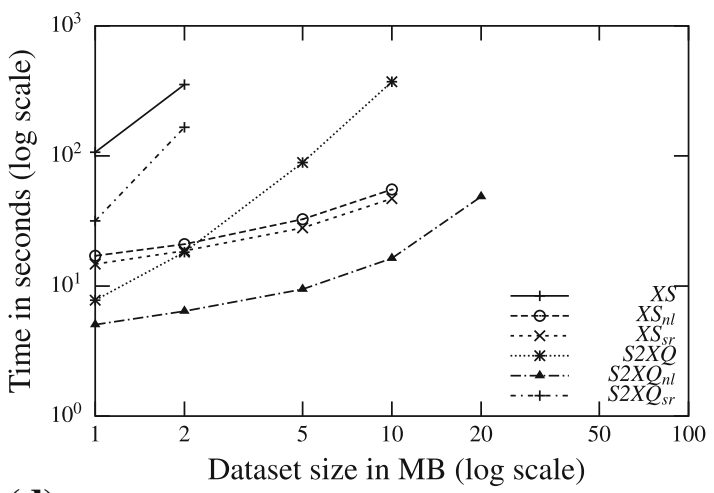

(d) Query $q_{11}^{\prime}$

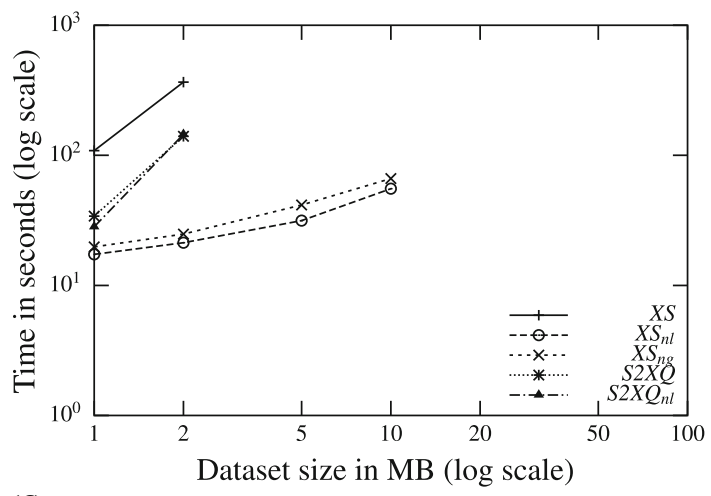

(f) Query $q_{11}^{\prime \prime}$

Fig. 16 Query response times for (variants of) $q_{10}$ and $q_{11}$ on all XMarkRDF datasets

tively reduces the overhead of using an external SPARQL engine for the evaluation of queries. Considering the $S 2 X Q_{s r}$ run, $o p t_{s r}$ produces no improvement in the query response times and in some cases $\left(q_{10}^{\prime}\right.$ and $q_{11}^{\prime}$ from Table 2) even deteriorates considerably the response times when compared with $S 2 X Q$. This further supports our previous claims that the XQuery engine is not capable of optimising the rewritten code from complex SPARQL queries.

Furthermore, the $S 2 X Q_{s r}$ runs could not evaluate the higher dataset sizes for query $q_{8}$, whose response times deteriorate considerably with the larger dataset sizes-as opposed to the $X S_{s r}$ runs which behaves consistently similar to $X S_{n l}$. This indicates that $S 2 X Q$ is not as efficient as the ARQ-based native SPARQL engine runs $X S_{s r}$ and $X S_{n l}$ for larger datasets.

We can draw similar conclusions for the opt $t_{n g}$ when comparing the query evaluation times of the opt $t_{s r}$ rewriting. However, the response times for this approach are deteriorated by the overhead of creating, inserting, and deleting the RDF Named Graph. This slowdown makes queries $q_{8}^{\prime \prime}$, $q_{10}^{\prime \prime}$ and $q_{11}^{\prime \prime}$ of the of the $o p t_{n l}$ rewriting outperform this optimisation. 
Table 2 Query response times in seconds of different optimisations for the $2 \mathrm{MB}$ XMarkRDF dataset. Optimisation not applicable $(n / a)$

\begin{tabular}{lrrlrlll}
\hline & \multicolumn{1}{c}{$X S$} & $S 2 X Q$ & $X S_{n l}$ & $S 2 X Q_{n l}$ & $X S_{s r}$ & $S 2 X Q_{s r}$ & $X S_{n g}$ \\
\hline$q_{8}$ & 358.27 & $\mathbf{3 . 4 4}$ & 15.66 & 3.54 & $n / a$ & $n / a$ & $n / a$ \\
$q_{9}$ & 355.71 & 18.91 & $\mathbf{1 5 . 2 0}$ & 19.35 & $n / a$ & $n / a$ & $n / a$ \\
$q_{10}$ & 35.89 & 178.71 & $\mathbf{1 9 . 7 8}$ & 156.09 & $n / a$ & $n / a$ & $n / a$ \\
$q_{11}$ & 371.46 & 17.99 & 22.67 & $\mathbf{6 . 4 3}$ & $n / a$ & $n / a$ & $n / a$ \\
$q_{8}^{\prime}$ & 355.63 & 3.71 & 15.48 & $\mathbf{2 . 8 7}$ & 10.60 & 3.35 & $n / a$ \\
$q_{9}^{\prime}$ & 357.13 & 19.16 & 15.12 & 19.10 & $\mathbf{1 1 . 7 9}$ & 15.44 & $n / a$ \\
$q_{10}^{\prime}$ & 36.73 & 180.32 & 18.24 & 154.95 & $\mathbf{1 6 . 3 7}$ & 199.28 & $n / a$ \\
$q_{11}^{\prime}$ & 354.20 & 18.21 & 21.00 & $\mathbf{6 . 4 0}$ & 18.63 & 165.99 & $n / a$ \\
$q_{8}^{\prime \prime}$ & 352.10 & 4.46 & 13.29 & $\mathbf{3 . 6 1}$ & $n / a$ & $n / a$ & 13.53 \\
$q_{9}^{\prime \prime}$ & 356.63 & 18.64 & 13.23 & 16.46 & $n / a$ & $n / a$ & $\mathbf{1 3 . 1 7}$ \\
$q_{10}^{\prime \prime}$ & 37.84 & 175.70 & $\mathbf{1 6 . 8 8}$ & 175.47 & $n / a$ & $n / a$ & 17.62 \\
$q_{11}^{\prime \prime}$ & 365.24 & 139.96 & $\mathbf{2 1 . 2 7}$ & 145.05 & $n / a$ & $n / a$ & 24.79 \\
\hline
\end{tabular}

\section{Related Work}

With the establishment of XML and RDF, tools and methods were introduced that rely on existing standards for retrieving and querying both languages. Most of the existing proposals to merge XML and RDF rely on translating the data from different formats and/or translating the queries from different languages. With this in mind, we divided the proposals into two categories: (1) Translation of data: these tools aim at integrating the heterogeneous data by translating between different formats, usually relying on user predefined mappings. (2) Integration of query languages: this category of approaches (where XSPARQL is also included) considers the integration and/or expansion of query languages to allow querying different formats. Next we give a short overview of some of the tools and proposals available in each category.

Data translation The TriX format [21] consists of an alternative serialisation for RDF in XML, with the aim of being compatible with standard XML tools. It uses XSLT as an extensibility mechanism, allowing to specify syntactic extensions and defining macros. $R 3 X^{17}$ uses an RDF processor and XSLT to transform RDF data into a predictable form of RDF/XML also catering for RSS. Similarly, Grit ${ }^{18}$ is designed to be a simplified normalisation for RDF, easier to process with XSLT than RDF/XML. Gloze [6] aims at directly interpreting an XML document as RDF data by providing transformations between XML and RDF based on the XML Schema definition. The transformation tries to map each XML element and attribute to an RDF property. The resulting transformation makes extensive use of RDF sequences to maintain the ordering from the XML structure. Droop et al. [28] translate the XML document into RDF,

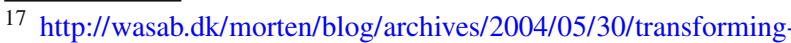
rdfxml-with-xslt.

$18 \mathrm{http} / / /$ code.google.com/p/oort/wiki/Grit.
}

annotating it with necessary information to answer XPath queries. The XPath queries are, in turn, translated into SPARQL queries, and the result of the execution of the SPARQL query is then translated into a format equivalent to the result of the XPath query.

Deursen et al. [26] present an approach for the transformation between XML and RDF in an ontology-dependent manner, introducing a language that allows to convert existing XML Schema documents (and XML documents conforming to the schemas) by defining mappings relating the schema to specified ontologies. Other approaches $[17,56]$ aim at translating an XML Schema into an equivalent OWL ontology. However, in our approach, we are focusing on translation and integration of instance data, rather than aiming at providing a semantic interpretation for XML data.

The approaches that propose a batch translation of data pose problems such as the replication of data and the need for constant synchronisation between the original data and the transformed data, for instance in the case of a frequently updated database. We argue that this approach is not optimal for most enterprise and Web scenarios and dynamic translations are the best way to describe and implement such integration of data.

Last, but not least, as we have also discussed XSPARQL as a means to transform between different RDF representations beyond the capabilities of SPARQL [53] in this paper, we should mention the forthcoming SPARQL 1.1 [38] specification, that will add features to SPARQL addressing such use cases (aggregation, value generation, etc.). Whereas no detailed studies of SPARQL 1.1's expressivity exist as of yet, we emphasise that XSPARQL — being a Turing-complete scripting language for RDF-will be able to encompass all features within SPARQL 1.1 and more.

Language integration Berrueta et al. [12] present a framework that allows to perform SPARQL queries from XSLT: XSLT+SPARQL. It relies on adding functions to XSLT that 
provide the ability to query SPARQL endpoints and uses standard XSLT to process the SPARQL XML results format. Similarly to our current implementation, they rely on a clear separation between the SPARQL query and XSLT parts of the query.

The following proposals suggest compiling a SPARQL query to XSLT/XQuery: Bikakis et al. [14] translate each SPARQL query into an XQuery using a previously defined mapping from OWL to XML Schema and [33] propose to embed SPARQL into XSLT or XQuery, presenting extensions to these languages to enable SPARQL querying where each SPARQL query is also translated into an equivalent XQuery. This language is very close to the XSPARQL language, but it does, however, require converting the RDF data to XML according to a predefined schema. Assuming the queried dataset is available this translation carries overhead into the query and in case the dataset is not available, for example due to being stored behind a SPARQL endpoint, such translation is not possible. Ding and Buxton presented an approach to translate SPARQL into XQuery at the 2011 Semantic Technology Conference. ${ }^{19}$ This rewriting generates XQuery specifically tailored for the Marklogic Server XML database engine. On a similar approach, integrating XPath into SPARQL [25], also promises to bridge the gap between $\mathrm{XML}$ and RDF. This is approach is similar to XSPARQL, although the choice here was to extend the SPARQL query language. While also catering for SQL queries, [31] presents a translation of SPARQL queries into XQuery and present encouraging benchmark results. Another similar approach is presented by [48], where the authors again translate SPARQL to XQuery by relying on a normal form of RDF/XML. Also according to our benchmarks, encoding SPARQL in XQuery seems a viable option-assuming that we have access to the RDF dataset beforehand-that would allow to compile XSPARQL to pure XQuery without the use of a separate SPARQL engine.

Zhou and $\mathrm{Wu}$ [59] propose another approach to represent RDF data as XML trees, based on translating RDFS into an XML Schema, and then translating SPARQL queries into XPath and XQuery queries.

RDF Twig [58] suggests XSLT extension functions that provide views on the sub-trees of an RDF graph. The main idea of RDF Twig is that while RDF/XML is hard to navigate using XPath, a subtree of an RDF graph can be serialised into a more useful form of RDF/XML. RDFXSLT ${ }^{20}$ provides an XSLT preprocessing stylesheet and a set of helper functions, similar to RDF Twig, yet implemented in pure XSLT 2.0, readily available for multiple platforms. The CORESE

\footnotetext{
$\overline{19} \mathrm{http} / / / \mathrm{semte}$ 2011.semanticweb.com/sessionPop.cfm?confid=62\& proposalid $=4015$.

${ }^{20}$ http://www.wsmo.org/TR/d24/d24.2/v0.1/20070412/rdfxslt.html.
}

framework ${ }^{21}$ also provides extensions of SPARQL to process XPath and XSL transformations in SPARQL queries and defines an extension to the XSLT language to allow to perform SPARQL queries.

Other approaches for querying heterogenous data were presented by Berger et al. [10]. This query language follows a different syntax than the W3C standardised SPARQL and XQuery and allows to write queries in the form of logical rules over an abstraction of the XML and RDF data models (represented as a graph).

The nSPARQL query language [50] proposes to extend SPARQL with navigational capabilities using nested regular expressions. With this addition, the language is sufficiently expressive to capture the semantics of RDFS. In addition to this, it introduces a number of graph navigation operators and adds the ability to selectively traverse the graph. This work is different than our current proposed approach for XSPARQL, but one of the possibilities for extending XSPARQL is to enable to perform XQuery enriched SPARQL queries.

Related to our nested queries optimisation, initial work has been presented by [5] over an extension of SPARQL that caters for nested queries and presented preliminary equivalences between types of nested queries with the aim of determining if query unnesting can be successfully applicable.

\section{Conclusion and Future Work}

In this paper we presented a novel query language, called XSPARQL, that combines XQuery and SPARQL to provide simplified transformations between the XML and RDF data models. We covered the semantics of XSPARQL, defined as an extension of the XQuery semantics, and presented our current implementation which consists of rewriting each XSPARQL query to an XQuery query. The implementation is available for download at http://xsparql.deri.org/ where we also provide an online XSPARQL query evaluator at http:// xsparql.deri.org/demo/.

We also presented different rewriting strategies for a particular category of XSPARQL queries, namely those containing nested expressions involving SPARQL queries and presented benchmark evaluation of these different rewritings. For these optimisations we detailed the rewriting functions describing their application in our current implementation of the XSPARQL language. We presented two types of optimisations for nested expressions: one based on reordering the expressions in the XQuery rewriting to minimise the number of calls to the SPARQL endpoint and another based on performing a more complex SPARQL query that takes care of joining the variables. The benchmarks carried out to determine the impact of our optimisations have shown encour-

$\overline{{ }^{21} \mathrm{http} / / / \mathrm{www}}$-sop.inria.fr/edelweiss/software/corese/. 
aging results, hinting on a large potential for optimisations in XSPARQL. Among the rewriting strategies presented in this paper and on our test data, pushing joins into a SPARQL engine appeared the most promising strategy. Our benchmark results showed that our optimisations are not only specific to XSPARQL having also improved the response times of the SPARQL2XQuery system to which we compared XSPARQL.

Future Work In this paper we have shown that nested queries can be efficiently evaluated by applying particular rewritings. Nonetheless all the tested rewriting strategies were created ad-hoc. A declarative algebra model would help to correctly and systematically study further optimisations for XSPARQL. As starting points, [34] and [36] have presented translations of XQuery to SQL, whereas in our own earlier works we have likewise translated SPARQL essentially to Relational Algebra [52]. These works seem to indicate valid starting points for further research on equivalences and optimisations in our language. Initial steps for defining such a declarative algebra can also be based on subsets of the XQuery language, for example XQuery core presented by Koch [43]. A proposal towards the declarative model of XSPARQL has been done by Bischof [16]. Although the initial set of optimisations proposed in this paper shows that our current implementation of performing interleaved calls to a SPARQL engine can be improved upon, a more tightly integrated implementation of the XSPARQL language should yield better results. Such an integrated implementation is planned for the near future where we can leverage optimisations proposed for SPARQL [37] or the proposed implementations of XQuery over relational databases [34,13]. Finally, the current XSPARQL language specification already allows to query data contained in XML and RDF datastores. However, updating data of these datastores is still not directly possible. We plan to extend the XSPARQL language to a full data manipulation language allowing to update, insert, and delete data contained in RDF tripestores. Here, similar to our combination of query languages, we will aim at combining common data manipulation languages for XML and RDF, such as SPARQL Update [32] and XQuery Update [55].

Acknowledgments This work was supported by SFI grant SFI/08/CE/I1380 (Líon-2), by an IRCSET scholarship, and by the Marie Curie IRSES Grant 24761 (Net2). We would like to thank Sven Groppe for his help with the SPARQL2XQuery system.

\section{A Example of XSPARQL Semantics Evaluation}

As an example we show the application of the XSPARQL evaluation semantics (presented in Sect. 4.2) to the sample query from Fig. 8b. The example query features both, the new SparqlForClause as well as the new ConstructClause.

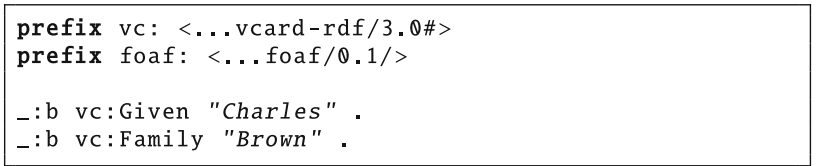

(a) Example input

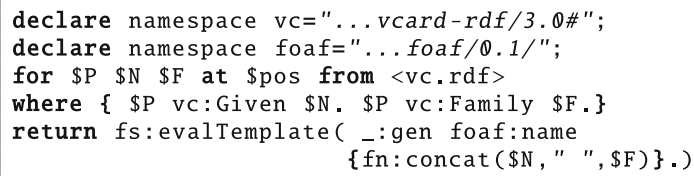

(b) Query after normalisation

Fig. 17 Example input and normalised query of Fig. 8

Table 3 Result of $f$ s:sparql

\begin{tabular}{lll}
\hline$\$ P$ & $\$ N$ & $\$ F$ \\
\hline :gen & "Charles" & "Brown" \\
\hline
\end{tabular}

We assume the input graph $v c . r d f$ as given in Fig. 17a. Let us go through the three phases of XQuery semantics evaluation, i.e., the normalisation, static type checking, and dynamic evaluation steps.

Normalisation In the normalisation step the SPARQLstyle namespace declarations are rewritten to XQuery namespace declarations (see Rule (N3)). After that, the whole construct query is rewritten to a SparqlForClauseby Rule (N8), the for * is expanded according to Rule (N6) and the resulting SparqlForClauseis then handled by Rule (N7). Rule $\llbracket \cdot \rrbracket_{\text {PosVar }}$ then adds a new positional variable (e.g., \$pos). Finally the construct is normalised by Rule (N9). The whole normalisation phase results in the query given in Fig. 17b.

Static Type Analysis By Rule (S2) the variables occurring in the WhereClause, namely $\$ P, \$ N$, and $\$ F$, are typed as RDFTerm, and the positional variable, \$pos, is typed as xs: integer. The whole SparqlForClause inherits its type from the contained return ExprSingle, which in turn inherits its type from the function $f$ s:evalCT which is RDFGraph.

Dynamic Evaluation First the new environment component activeDataset is changed from empty to the one given in the DatasetClause, i.e., the graph contained in vc.rdf. According to Rule (D3) the WhereClause is evaluated using the $f$ s:sparql function with the active dataset, as just initialised, the WhereClause as given in the query, and empty SolutionModifiers. The fs:sparql function call results in a sequence of PatternSolutions (in this case a singleton solution) as given in Table 3. Next, the same rule extracts the variable bindings for all variables, using the $f$ s: value function, and assigns them to the corresponding XQuery variables, typed as RDFTerm. After that the return expression ExprSingle is evaluated, using the just initialised variables. The 


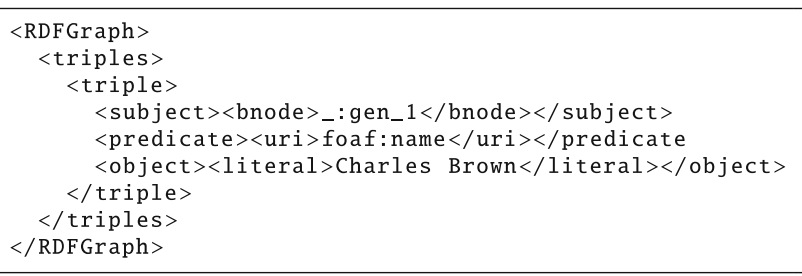

Fig. 18 Query results

$f_{s}$ :evalCT function calls the $f$ s:validTriple function passing it a blank node generated by the $f s$ :bnode function as subject, "foaf: name" as predicate and the result of fn:concat as object. The $f s:$ bnode function (as given by Rule (D7)) generates a fresh blank node label for each element of the Patternsolution. For this example we assume that the function returns the new blank node label "_:gen_1". The $f s$ :validTriple function tests these three values for validity. Since the subject is of type bnode, the predicate is a QName (and therefore considered as being of type uri), and the object is of type literal, namely an xs: string, the function returns them as a valid RDFTriple. The $f_{\text {s:evalCT func- }}$ tion eventually returns the result of the single $f$ s:validTriple function call and thus the result of the whole query as an element of type RDFGraph as shown in Fig. 18. Serialised to Turtle the query result, including QName expansion, is the expression_:gen_1 <http://..ffoaf/0.1/name> "Charles Brown".

\section{B Implementation Functions Example}

Figure 19 presents some of the XQuery functions defined in the XSPARQL language implementation, namely to correctly format RDF terms (Fig. 19a) and to validate triples resulting from a construct expression (Fig 19b).

\section{Proofs}

\section{C.1 Proof for Proposition 1}

\section{Proposition 1 XSPARQL is a conservative extension of} XQuery.

Proof We show that the additional rules introduced in Sect. 4.2 do not modify the semantics of any native XQuery. The XSPARQL semantics-expressed in terms of normalisation rules, static typing rules, and dynamic evaluation rules-strictly extend the native semantics of XQuery. In the semantics definition we also define new environment components, namely statEnv.posVars and dynEnv.activeDataset, which are not used in the XQuery semantics and thus do not interfere with query evaluation. However, for the XSP-

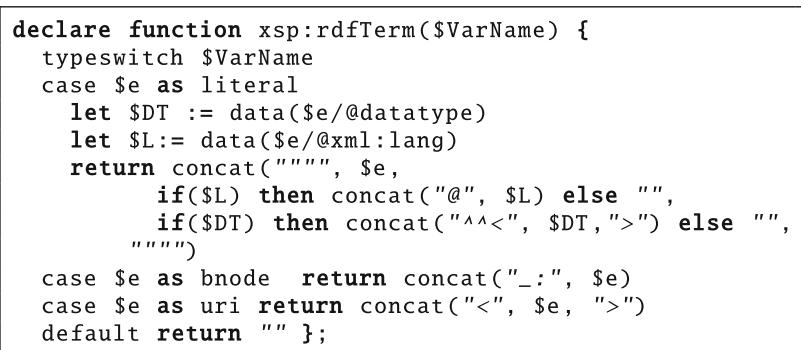

(a) xsp:rdfTerm function

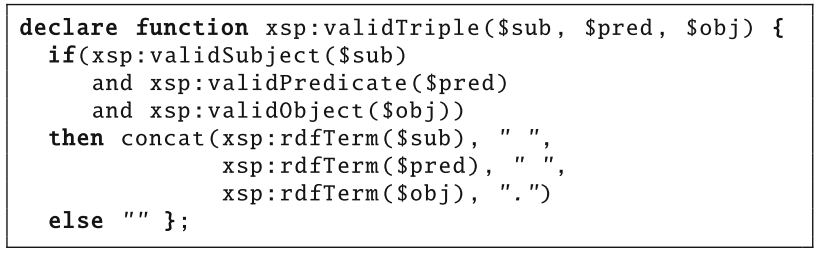

(b) xsp:validTriple function

Fig. 19 Implementation functions

ARQL semantics we also extend the normalisation rules and static analysis rules for native XQuery for clauses. More specifically, rule (N5) extends the XQuery for normalisation by adding a new variable to each position-variable free for expression (i.e., that does not have an at clause). As stated these new position variables are disjoint from the variables in scope, and thus this rewriting does not interfere with the semantics of the original query. The only rules which use the newly created position variables are (i) the slightly modified static type analysis rule (S3) which extends the XQuery for static analysis rule by collecting the position variables in the static environment component statEnv.posVars, thus also maintaining the original semantics of the original XQuery for rule and (ii) the dynamic evaluation rule (D7) which accesses statEnv.posVars to generate Skolem-identifiers for blank nodes in construct parts. However, rule (D7) only applies to XSPARQL queries which fall outside the native XQuery fragment, whereas the semantics of native XQuery queries remains untouched and independent of the extra environment components in XSPARQL.

\section{C.2 Proof for Lemma 1}

Lemma 1 Given a graph pattern $P$, a dataset $D$ and the $X S P A R Q L$ instance mapping $\mu_{C}$ of the expression context $C$ over which $P$ is evaluated, let $\Omega_{1}=\operatorname{eval}_{x s}\left(D, P, \mu_{C}\right)$ and $\Omega_{2}=\operatorname{eval}(D, P)$ be solution mappings. If $\operatorname{vars}(P) \cap$ $\operatorname{dom}\left(\mu_{C}\right)=\emptyset$, then $\Omega_{1}=\Omega_{2} \bowtie\left\{\mu_{C}\right\}$.

Proof The XSPARQL BGP matching, eval ${ }_{x s}\left(D, P, \mu_{C}\right)$, extends SPARQL's BGP matching, eval $(D, P)$, by defining that the solutions of the BGP are the ones compatible with the XSPARQL instance mapping $\mu_{C}$. Since the evalu- 
ation of graph patterns (such as union, optional, graph, and filter) remains unchanged from the SPARQL semantics let us focus on the evaluation of a BGP $P$. If there are no shared values between the graph pattern and the XSPARQL instance mapping, $\operatorname{vars}(P) \cap \operatorname{dom}\left(\mu_{C}\right)=\emptyset$, then each solution $\mu \in \Omega_{2}$ returned by the SPARQL BGP evaluation semantics is trivially compatible with $\mu_{C}$ and the result of the XSPARQL BGP matching is $\mu \cup \mu_{C}$. Extending this result to all solution mappings in $\Omega_{2}$, we obtain that $\Omega_{1}=\Omega_{2} \bowtie\left\{\mu_{C}\right\}$.

\section{C.3 Proof for Proposition 2}

Proposition 2 XSPARQL is a conservative extension of SPARQL construct queries.

Proof For XSPARQL queries consisting of a SPARQL construct query, there cannot exist any previous bindings for variables in XSPARQL and thus the XSPARQL instance mapping $\mu_{C}$ over which the construct query will be executed is empty. Let $P$ represent the graph pattern of the construct query and $D$ the dataset, trivially there are no shared variables between $\mu_{C}$ and $P$ and so, following Lemma 1 the bindings $\Omega_{1}$ for XSPARQL BGP matching are the same bindings $\Omega_{2}$ as SPARQL BGP matching, since $\Omega_{1}=\Omega_{2} \cup\{\emptyset\}$ and hence $\Omega_{1}=\Omega_{2}$. Furthermore, the formal semantics function $f$ s:evalTemplate returns an RDF graph satisfying all the conditions of Definition 11: (1) Ignoring invalid RDF triples-Item 1-is guaranteed by Rules D5 and D6; and (2) The generation of distinct blank nodes for each solution sequence-Item 2-is enforced by the blank node skolemisation rules (Rules (D7) and (D8)).

\section{C.4 Proof for Lemma 2}

Lemma 2 Let $P$ be a BGP, D a dataset, and $\mu$ the XSPARQL instance mapping of $P$. Considering $P^{\prime}=\mu(P)$, we have that $\operatorname{eval}_{x s}(D, P, \mu)=\operatorname{eval}\left(D, P^{\prime}\right) \bowtie\{\mu\}$.

Proof Since, according to the variable substitution operation we have that $\operatorname{vars}\left(P^{\prime}\right)=\operatorname{vars}(P) \backslash \operatorname{dom}(\mu)$, we also have that $\operatorname{vars}\left(P^{\prime}\right) \cap \operatorname{dom}(\mu)=\emptyset$, and it follows directly from Lemma 1 that $\operatorname{eval}_{x s}(D, P, \mu)=\operatorname{eval}\left(D, P^{\prime}\right) \bowtie\{\mu\}$.

\section{C.5 Proof for Proposition 3}

Proposition 3 Let $Q$ be a SparqlForClauseofform (Q1) and dynEnv the dynamic environment of $Q$, then dynEnv $\vdash Q \Rightarrow$ Val if and only if dynEnv $\vdash \operatorname{tr}(Q) \Rightarrow$ Val.
Proof $(\Leftarrow)$ Let us show that if dynEnv $\vdash \operatorname{tr}(Q) \Rightarrow$ Val then dynEnv $\vdash Q \Rightarrow$ Val. The evaluation of $Q$ consists of the application of Rule (D1) as

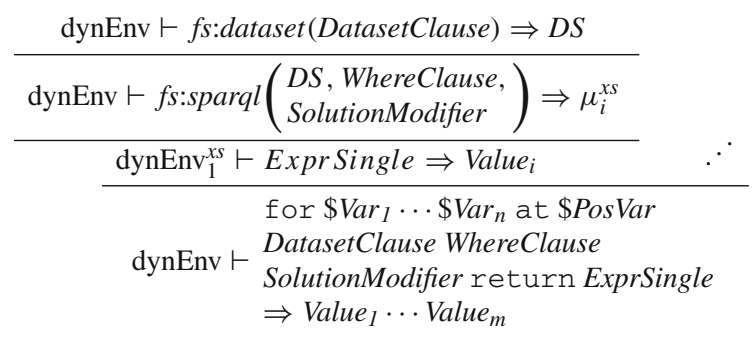

where, for each $\mu_{i}^{x s}$,

$$
\begin{aligned}
\operatorname{dynEnv}_{1}^{x s}=\operatorname{dynEnv} & +\operatorname{activeDataset}(D S) \\
& +\operatorname{varValue}\left(\begin{array}{l}
\text { PosVar } \Rightarrow i \\
\operatorname{Var}_{l} \Rightarrow f s: \operatorname{value}\left(\mu_{i}^{x s}, \operatorname{Var}_{I}\right) \\
\cdots ; \\
\operatorname{Var}_{n} \Rightarrow f s: \operatorname{value}\left(\mu_{i}^{x s}, \operatorname{Var}_{n}\right)
\end{array}\right)
\end{aligned}
$$

Let $\mu_{C}$ be the XSPARQL instance mapping of the expression context that includes dynEnv and $\Omega_{t r}$ the pattern solution resulting from evaluating the xsp:sparqlcall function, i.e., $\Omega_{t r}=\operatorname{eval}($ DatasetClause, $P$ ), where $P$ is the rewriting of WhereClause according to $\mu_{C}$. Furthermore, let $\mu_{i} \in \Omega_{t r}$ be the solution mapping from which Val is generated, i.e., there exists some dynamic environment dynEnv ${ }^{t r}$ based on dynEnv and extended with the variable bindings from $\mu_{i}$ such that dynEnv ${ }^{t r} \vdash$ ExprSingle $\Rightarrow$ Val.

Consider $\Omega_{x s}=$ eval ${ }_{x s}$ (DatasetClause, WhereClause, $\mu_{C}$ ) as the solution sequence resulting from the evaluation of the $f$ s:sparql function. As we know from Lemma $2, \Omega_{x s}=$ $\Omega_{t r} \bowtie\left\{\mu_{C}\right\}$ and thus there must exist a solution mapping $\mu_{x s} \in \Omega_{x s}$ such that $\mu_{x s}=\mu_{i} \bowtie \mu_{C}$. From (T1) we infer that there exists a dynamic environment dynEnv ${ }^{x s}$ that results from extending dynEnv with the variable bindings from $\mu_{x s}$ and thus this environment will also contain all the variable mappings from dynEnv ${ }^{t r}$. Since we know that dynEnv ${ }^{t r} \vdash$ ExprSingle $\Rightarrow \mathrm{Val}$, we also have that dynEnv $^{x s} \vdash$ ExprSingle $\Rightarrow$ Val and thus dynEnv $\vdash Q \Rightarrow$ Val.

$(\Rightarrow)$ Next we will show that if dynEnv $\vdash Q \Rightarrow V a l$ then dynEnv $\vdash \operatorname{tr}(Q) \Rightarrow V a l$. We present the proof tree for each of the XQuery core expressions in the $\operatorname{tr}(Q)$ rewriting. The proof trees are presented for each line of the $\operatorname{tr}(Q)$ rewriting and, in each proof tree, Expr corresponds to the XQuery expressions of the following lines: 
- let expression of line (1):

$$
\begin{gathered}
\text { dynEnv } \vdash \text { xsp: sparq1Cal1 }\left(\begin{array}{l}
\text { select Vars } \\
\text { DatasetClause } \\
\text { WhereClause } \\
\text { SolutionModifier }
\end{array}\right) \Rightarrow \Omega_{t r} \\
\text { dynEnv }{ }_{1}^{\text {tr }} \vdash \text { Expr } \Rightarrow \text { Res } \\
\text { dynEnv } \vdash \quad \text { xsp: sparqlCal1 }\left(\begin{array}{l}
\text { select Vars } \\
\text { DatasetClause } \\
\text { WhereClause } \\
\text { SolutionModifier }
\end{array}\right) \\
\text { return Expr } \Rightarrow \text { Res }
\end{gathered}
$$

where

$$
\text { dynEnv }_{1}^{t r}=\text { dynEnv }+\operatorname{varValue}\left(\mathrm{xsp}: \text { results } \Rightarrow \Omega_{t r}\right)
$$

- for expression of line (2)

$$
\begin{aligned}
& \text { dynEnv }_{1}^{t r} \vdash \text { \$xsp:results//sr:result } \Rightarrow \mu_{i} \\
& \frac{\text { dynEnv }_{2}^{t r} \vdash \operatorname{Expr} \Rightarrow \operatorname{Res}_{i}}{\text { for \$xsp:result at \$PosVar }} \\
& \text { where return } \operatorname{Exp} \Rightarrow \operatorname{Res}_{1}, \cdots, \operatorname{Res}_{n}
\end{aligned}
$$$$
\text { dynEnv }_{2}^{t r}=\operatorname{dynEnv}_{1}^{t r}+\operatorname{varValue}\left(\begin{array}{l}
\text { xsp:result } \Rightarrow \mu_{i} ; \\
\text { PosVar } \Rightarrow i
\end{array}\right)
$$

- let expressions of lines (3)-(4)

Here we consider all the let expressions represented by line (3), where $\$ v \in$ Vars:

$$
\begin{gathered}
\frac{\text { dynEnv }_{2}^{t r} \vdash \$ \text { xsp:result/sr:binding }[@ \text { name }=v] / * \Rightarrow V}{\operatorname{dynEnv}_{3}^{t r} \vdash \text { ExprSingle } \Rightarrow \text { Res }} \\
\text { dynEnv }_{2}^{t r} \vdash \begin{array}{c}
\text { let } \$ v:= \\
\$ x \text { sp:result } / \text { sr:binding }[@ \text { name }=v] / * \\
\text { return ExprSingle } \Rightarrow \text { Res }
\end{array}
\end{gathered}
$$

where dynEnv ${ }_{3}^{t r}=\operatorname{dynEnv}_{2}^{t r}+\operatorname{varValue}(v \Rightarrow V)$

Consider the dynamic environment dynEnv ${ }_{i}^{x s}$ such that dynEnv $_{i}^{x s} \vdash$ ExprSingle $\Rightarrow$ Val where, as we know from (T1), dynEnv ${ }_{i}^{x s}$ extends dynEnv by changing the activeDataset and varValue environment components.

Consider $\mu_{C}, \Omega_{x s}$, and $\Omega_{t r}$ as before. From Lemma 2 we get that $\Omega_{x s}=\Omega_{t r} \bowtie\left\{\mu_{C}\right\}$ and since $\mu_{C}$ is created based on dynEnv.varValue, all the variable bindings from $\mu_{C}$ are already included in dynEnv.

From the proof trees of $\operatorname{tr}(Q)$ we can see that the for expression from line (2) iterates over the all the solution mappings included in $\Omega_{t r}$ and the let expressions from lines (3) and (4) ensure there exists a dynEnv ${ }_{2}^{t r}$ such that dynEnv ${ }_{2}^{t r}$.varValue contains all the variable bindings from dynEnv ${ }_{i}^{x s}$. varValue, and we have that dynEnv ${ }_{2}^{t r} \vdash$ ExprSingle $\Rightarrow$ Val.

\section{C.6 Proof for Proposition 4}

Proposition 4 Let $Q$ be a XSPARQL expression ofform (Q2) or (Q3) and dynEnv the dynamic environment of $Q$, then dynEnv $\vdash Q \Rightarrow$ Val if and only ifdynEnv $\vdash$ opt $_{n l}(Q) \Rightarrow$ Val.
Proof We now present the proof of the $o p t_{n l}$ rewriting function for expressions of the form (Q3).

We start by showing the proof for the base case, where ExprSingle of (Q3) does not contain any occurrences of (Q3).

Base Case. $(\Rightarrow)$ We start by showing that if dynEnv $\vdash Q \Rightarrow$ Val then dynEnv $\vdash o p t_{n l}(Q) \Rightarrow$ Val. Consider $\Omega_{x s}^{o u t}$ and $\Omega_{x s}^{i n}$ the solution sequences returned, respectively, by the evaluation of the outer and inner SparqlForClauses of $Q$ and the set of join variables $J=\operatorname{Vars}^{\text {out }} \cap \operatorname{vars}\left(\right.$ WhereClause $\left.^{\text {in }}\right)$. Furthermore, consider $\mu_{x s}^{\text {out }} \in \Omega_{x s}^{\text {out }}$ and $\mu_{x s}^{\text {in }} \in \Omega_{x s}^{\text {in }}$ the solution mappings that agree on the value of each join variable $j \in J$ from where $\mathrm{Val}$ is generated, i.e., there exists some dynamic environment dynEnv ${ }^{x s}$ based on dynEnv and extended with the variable mappings from $\mu_{x s}^{\text {out }}$ and $\mu_{x s}^{\text {in }}$ such that dynEnv ${ }^{x s} \vdash$ ExprSingle $\Rightarrow$ Val.

We show now the proof tree for each of the XQuery core expressions in the $\operatorname{opt}_{n l}(Q)$ rewriting where, in each proof tree, Expr corresponds to the XQuery expressions of the following lines:

- let expression of line (1), considering Vars $=\operatorname{Vars}^{i n} \cup$ (Vars $^{\text {out }} \cap \operatorname{vars}\left(\right.$ WhereClause $\left.e^{i n}\right)$ ), we have that

$$
\begin{aligned}
& \text { dynEnv } \vdash \mathrm{xsp}: \text { sparqlCal1 }\left(\begin{array}{l}
\text { select Vars } \\
\text { DatasetClause } \\
\text { WhereClause }^{\text {in }} \\
\text { SolutionModifier }^{\text {in }}
\end{array}\right) \Rightarrow \Omega^{\text {in }} \\
& \frac{\operatorname{dynEnv}_{1}^{n l} \vdash \operatorname{Expr} \Rightarrow \operatorname{Res}}{\text { let } \$ \mathrm{xsp} \cdot \mathrm{res} \text { in }:=} \\
& \text { dynEnv } \vdash \text { xsp:sparqlCall }\left(\begin{array}{l}
\text { select Vars } \\
\text { DatasetClause } \\
\text { WhereClause }^{\text {in }} \\
\text { SolutionModifier }
\end{array}\right) \\
& \text { return } \operatorname{Exp} r \Rightarrow \text { Res }
\end{aligned}
$$

where

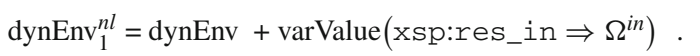

The function $\operatorname{opt}_{n l}(Q)$ translates the SparqlForClause from lines (4)-(6) of $Q$ into the xsp:sparqlcall of line (1). The inner SparqlForClause of $Q$ is evaluated considering some dynamic environment dynEnv ${ }_{i}^{x s}$ (and its expression context $C_{i}$ ). Since dynEnv ${ }_{i}^{x s}$ is an extension of dynEnv we have that $\operatorname{dom}\left(\mu_{C}\right) \subseteq \operatorname{dom}\left(\mu_{C_{i}}\right)$. The rewritten $\mathrm{xsp}$ : sparqlCall function is evaluated over the dynamic environment dynEnv (included in expression context $C$ ). Consider $\mu_{C}$ the XSPARQL instance mapping of $C$ and $\mu_{C_{i}}$ the XSPARQL instance mapping of $C_{i}$. Let $\Omega_{x s}^{i n}=$ eval $_{x s}\left(\right.$ DatasetClause $^{i n}$, WhereClause $\left.^{i n}, \mu_{C_{i}}\right)$ be the solution sequence resulting from the evaluation of the inner SparqlForClause of $Q$ and the solution sequence resulting from evaluating the $\mathrm{xsp}: \mathrm{sparqlCall}$ function be $\Omega_{n l}^{i n}=\operatorname{eval}\left(\right.$ DatasetClause $\left.{ }^{i n}, P^{i n}\right)$, where $P^{\text {in }}$ 
is the graph pattern obtained from replacing the variables in WhereClause ${ }^{i n}$ according to $\mu_{C}$. As $\operatorname{dom}\left(\mu_{C}\right) \subseteq$ $\operatorname{dom}\left(\mu_{C_{i}}\right)$, i.e., $\mu_{C}$ contains less bindings for variables than $\mu_{C_{i}}$, the rewritten graph pattern $P_{i n}$ contains more unbound variables, and we get that $\Omega_{x s}^{i n} \preceq \Omega_{n l}^{i n}$.

- let expression of line (2)

$$
\begin{aligned}
& \operatorname{dynEnv}_{1}^{n l} \vdash \mathrm{xsp}: \operatorname{sparq1Cal1}\left(\begin{array}{l}
\text { select } \text { Vars }^{\text {out }} \\
\text { DatasetClause }^{\text {out }} \\
\text { WhereClause }^{\text {out }} \\
\text { SolutionModifier }
\end{array}\right) \\
& \Rightarrow \Omega^{\text {out }} \\
& \begin{aligned}
\hline \operatorname{dynEnv}_{2}^{n l} \vdash \operatorname{Exp} r & \Rightarrow \text { Res } \\
\hline \text { let \$xsp:res_out }:= & \left(\begin{array}{l}
\text { select Vars }{ }^{\text {out }} \\
\text { DatasetClause } \\
\text { WhereClause }^{\text {out }} \\
\text { SolutionModifier }^{\text {out }}
\end{array}\right)
\end{aligned} \\
& \text { where return Expr } \Rightarrow \text { Res }
\end{aligned}
$$

$$
\operatorname{dynEnv}_{2}^{n l}=\operatorname{dynEnv}_{1}^{n l}+\operatorname{varValue}\left(x \text { sp:res_out } \Rightarrow \Omega^{\text {out }}\right) \text {. }
$$

Regarding the SparqlForClause of lines (1)-(3) of $Q$ (evaluated considering dynEnv), the $\operatorname{opt}_{n l}(Q)$ translates it into the xsp:sparqlCall from line (2), which is evaluated over dynEnv ${ }_{1}^{n l}$.

Consider $C_{1}$ the expression context where dynEnv ${ }_{1}^{n l}$ is included, $\mu_{C_{1}}$ the XSPARQL instance mapping of $C_{1}$ and $P^{\text {out }}$ the graph pattern obtained from replacing the variables in WhereClause out according to $\mu_{C_{1}}$. From (T3) we can see that $\operatorname{dom}\left(\mu_{C_{1}}\right)=\operatorname{dom}\left(\mu_{C}\right) \cup\{\$ x$ sp:res_in $\}$, but \$xsp:res_in belongs to the \$xsp: reserved namespace so it will not be included in the variables of WhereClause out, and we can observe that we obtain the same graph pattern $P^{\text {out }}$ by replacing WhereClause $^{\text {out }}$ according to $\mu_{C}$. Let $\Omega_{x s}^{\text {out }}=$ eval $_{x s}\left(\right.$ DatasetClause ${ }^{\text {out }}$, WhereClause ${ }^{\text {out }}, \mu_{C}$ ) be the solution sequence resulting from evaluating the outer SparqlForClause according to XSPARQL semantics and $\Omega_{n l}^{\text {out }}=\operatorname{eval}\left(\right.$ DatasetClause $\left.{ }^{\text {out }}, P^{\text {out }}\right)$ be the pattern solution resulting from evaluating the rewritten outer SparqlForClause according to SPARQL semantics. Following Lemma 2, we have that $\Omega_{x s}^{\text {out }}=\Omega_{n l}^{\text {out }} \bowtie\left\{\mu_{C}\right\}$ and, as we have seen from the proof of Proposition 3, since $\mu_{C}$ is already included in dynEnv, we have that $\Omega_{x s}^{\text {out }}=\Omega_{n l}^{\text {out }}$.

- for expression of line (3)

$$
\begin{aligned}
& \text { dynEnv }_{2}^{n l} \vdash \text { \$xsp:res_out } / / \text { sr:result } \Rightarrow \mu_{i} \\
& \frac{\operatorname{dynEnv}_{3}^{n l} \vdash \operatorname{Expr} \Rightarrow \operatorname{Res}_{i}}{\text { for } \$ \mathrm{xsp}: \text { rout at } \$ \text { PosVar }^{\text {out }}} \\
& \text { dynEnv }_{2}^{n l} \vdash \text { in \$xsp:res_out//sr:result } \\
& \text { return } \operatorname{Expr} \Rightarrow \operatorname{Res}_{1}, \cdots, \operatorname{Res}_{n}
\end{aligned}
$$

where

$$
\operatorname{dynEnv}_{3}^{n l}=\operatorname{dynEnv}_{2}^{n l}+\operatorname{varValue}\left(\begin{array}{l}
\text { xsp:rout } \Rightarrow \mu_{i} ; \\
\text { PosVar }{ }^{\text {out }} \Rightarrow i
\end{array}\right)
$$

- let expressions of line (4)

Here we consider all the let expressions represented by line (4), where $\$ v \in$ Vars $^{\text {out }}$ :

$$
\begin{aligned}
& \text { dynEnv }_{3}^{n l} \vdash \text { Xxsp:rout/sr:binding }[@ \text { name }=v] / * \Rightarrow V
\end{aligned}
$$

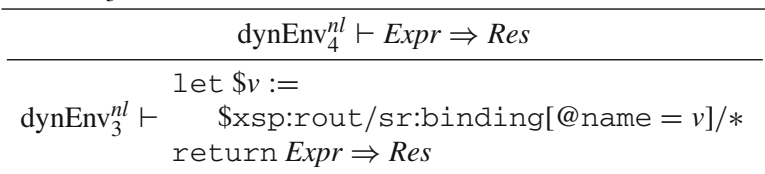

where

$$
\operatorname{dynEnv}_{4}^{n l}=\operatorname{dynEnv}_{3}^{n l}+\operatorname{varValue}(v \Rightarrow V) .
$$

- for expression of line (5)

$$
\begin{aligned}
& \text { dynEnv }_{4}^{n l} \vdash \text { \$xsp:res_in//sr:result } \Rightarrow S_{i} \\
& \frac{\text { dynEnv }_{5}^{n l} \vdash \operatorname{Expr} \Rightarrow \text { Res }_{i}}{\text { for \$xsp:rin at \$PosVar }} \\
& \text { dynEnv }_{4}^{n l} \vdash \text { in \$xsp:res_in//sr:result } \\
& \text { return } \operatorname{Expr} \Rightarrow \operatorname{Res}_{1}, \cdots, \operatorname{Res}_{n}
\end{aligned}
$$

where

$$
\operatorname{dynEnv}_{5}^{n l}=\operatorname{dynEnv}_{4}^{n l}+\operatorname{varValue}\left(\begin{array}{l}
\text { xsp:rin } \Rightarrow S_{i} ; \\
\operatorname{Pos} V a r^{i n} \Rightarrow i
\end{array}\right)
$$

- if expression of lines (6)-(9)

$$
\begin{aligned}
& \operatorname{dynEnv}_{5}^{n l} \vdash \operatorname{join}_{s r}\left(\begin{array}{l}
\text { Vars }^{\text {out }} \cap \operatorname{vars}(\text { WhereClause }), \\
\text { \$xsp:res_out, \$xsp:res_in }
\end{array}\right) \Rightarrow \text { true } \\
& \text { dynEnv }_{5}^{n l} \vdash \text { ExprSingle } \Rightarrow \text { Res }_{1} \\
& \begin{aligned}
& \operatorname{dynEnv}_{5}^{n l} \vdash \text { if }\left(\operatorname{join}_{s r}\left(\begin{array}{l}
\text { Vars } \\
\text { \$xsp:res_out, } \cap \text { vars }(\text { WhereClause }), \\
\text { then ExprSingle else }() \Rightarrow \text { Res }_{1}
\end{array}\right)\right) \\
& \text { then }
\end{aligned}
\end{aligned}
$$

- let expressions of line (7) and (8)

Here we consider all the let expressions represented by line (7), where $\$ v \in$ Vars $^{\text {out }} \triangle$ vars $\left(\right.$ WhereClause $e^{\text {in }}$ ).

$$
\frac{\text { dynEnv }_{5}^{n l} \vdash \text { \$xsp:res_in/sr:binding }[@ \text { name }=v] / * \Rightarrow V}{\text { dynEnv }_{6}^{n l} \vdash \text { ExprSingle } \Rightarrow \text { Res }}
$$

where

$$
\operatorname{dynEnv}_{6}^{n l}=\operatorname{dynEnv}_{5}^{n l}+\operatorname{varValue}(v \Rightarrow V) .
$$

Since we know that $\Omega_{n l}^{\text {out }}=\Omega_{x s}^{\text {out }}$ and $\Omega_{x s}^{\text {in }} \preceq \Omega_{n l}^{\text {in }}$, we obtain that $\mu_{x s}^{\text {out }} \in \Omega_{n l}^{\text {out }}$ and $\mu_{x s}^{\text {in }} \in \Omega_{n l}^{\text {in }}$. Since $\operatorname{opt}_{n l}(Q)$ performs a nested loop iteration over $\Omega_{n l}^{\text {out }}$ and $\Omega_{n l}^{\text {in }}$, the join ${ }_{s r}$ function 
will join the two solution mappings successfully since $\mu_{x s}^{\text {out }}$ and $\mu_{x s}^{i n}$ share the same values for the join variables, and thus we have that dynEnv $\vdash$ opt $_{n l}(Q) \Rightarrow$ Val.

$(\Leftarrow)$ We now proceed by showing that if dynEnv $\vdash$ $o p t_{n l}(Q) \Rightarrow V a l$, then dynEnv $\vdash Q \Rightarrow V a l$. Let $\Omega_{n l}^{\text {out }}$ and $\Omega_{n l}^{i n}$ be the pattern solutions returned by the outer and inner SparqlForClauses, respectively, and let $\mu_{n l}^{\text {out }} \in \Omega_{n l}^{\text {out }}$ and $\mu_{n l}^{i n} \in \Omega_{n l}^{i n}$ be the solution mappings, where Val is deduced from, i.e., $\mu_{n l}^{\text {out }}$ and $\mu_{n l}^{\text {in }}$ agree on their values for the join variables. We also know that there must exist a dynamic environment dynEnv ${ }^{n l}$, based on dynEnv and extended with the variable mappings $\mu_{n l}^{\text {out }}$ and $\mu_{n l}^{\text {in }}$ such that dynEnv ${ }^{n l} \vdash$ ExprSingle $\Rightarrow$ Val.

Let us turn to the evaluation of dynEnv $\vdash Q \Rightarrow$ Val.

- SparqlForClause from lines (1)-(3), where Expr corresponds to the SparqlForClause from lines (4)-(6) of $Q$. The evaluation of this SparqlForClause consists of the application of Rule (D1):

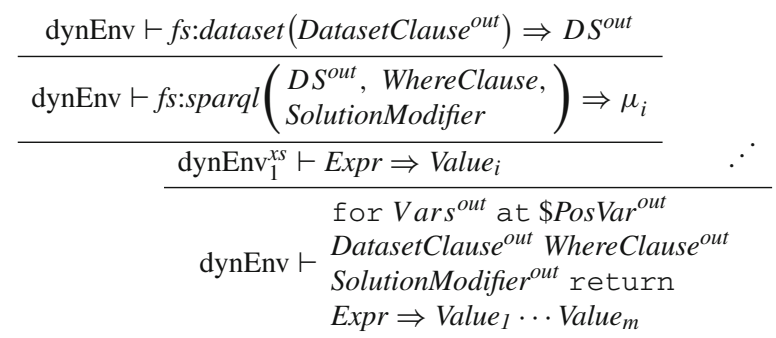

with $\operatorname{Vars}^{\text {out }}=\$ \operatorname{Var}_{1}^{\text {out }} \cdots \$ \operatorname{Var}_{n}^{\text {out }}$, we have for each $\mu_{i}$

$$
\begin{aligned}
& \text { dynEnv }+ \text { activeDataset }\left(D S^{\text {out }}\right) \\
& \operatorname{dynEnv}_{1}^{x s}=+\operatorname{varValue}\left(\begin{array}{l}
\text { PosVar }{ }^{\text {out }} \Rightarrow i ; \\
\operatorname{Var}_{1}^{\text {out }} \Rightarrow f \text { s:value }\left(\mu_{i}, \operatorname{Var}_{1}^{\text {out }}\right) ; \\
\ldots ; \\
\operatorname{Var}{ }_{n}^{\text {out }} \Rightarrow f \text { s:value }\left(\mu_{i}, \operatorname{Var}_{n}^{\text {out }}\right)
\end{array}\right)
\end{aligned}
$$

- SparqlForClause of lines (4)-(6):

The evaluation of dynEnv ${ }_{1}^{x s} \vdash E x p r \Rightarrow$ Value $_{i}$ is given by

$$
\begin{aligned}
& \operatorname{dynEnv}_{1}^{x s} \vdash f_{s} \text { dataset }\left(\text { DatasetClause } e^{i n}\right) \Rightarrow D S^{i n}
\end{aligned}
$$

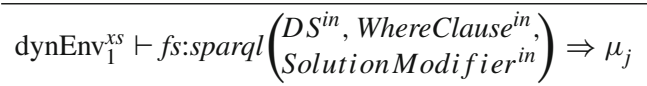

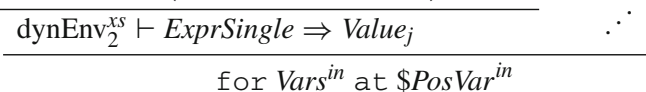

$$
\begin{aligned}
& \operatorname{dynEnv}_{1}^{x s} \vdash \begin{array}{l}
\text { DatasetClause } e^{\text {in }} \text { WhereClause }^{\text {in }} \\
\text { SolutionModifier in return }
\end{array} \\
& \text { ExprSingle } \Rightarrow \text { Value }_{1} \text {... } \text { Value }_{m}
\end{aligned}
$$

where, considering $\operatorname{Vars}^{\text {in }}=\$ \operatorname{Var}_{1}^{i n} \ldots \$ \operatorname{Var}_{n}^{\text {in }}$, we have for each $\mu_{j}$

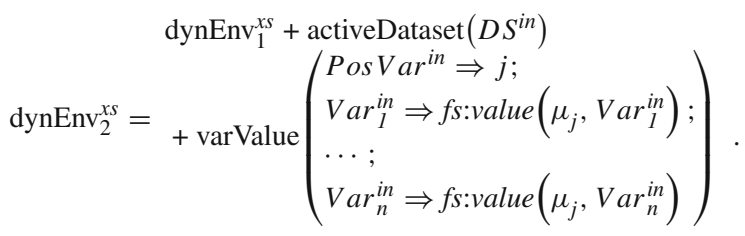

As we know from the $(\Rightarrow)$ direction of the proof, $\Omega_{n l}^{o u t}=$ $\Omega_{x s}^{\text {out }}$ and so we have that $\mu_{n l}^{\text {out }} \in \Omega_{x s}^{\text {out }}$. Regarding the evaluation of the inner SparqlForClausewe have that $\Omega_{x s}^{i n} \preceq \Omega_{n l}^{i n}$. We consider two cases: (i) $\mu_{n l}^{i n} \in \Omega_{x s}^{i n}$ or (ii) $\mu_{n l}^{i n} \notin \Omega_{x s}^{i n}$. In (i), we immediately get the desired result that dynEnv $\vdash Q \Rightarrow \mathrm{Val}$. For (ii), consider $\mu_{C_{I}}^{x s}$ the XSPARQL instance of the inner SparqlForClause (created based on dynEnv ${ }_{1}^{x s}$ ). As we can see from (T4), dynEnv ${ }_{1}^{x s}$ (and thus also $\mu_{C_{l}}^{x s}$ ) includes the bindings for variables from each solution mapping $\mu_{i} \in \Omega_{x s}^{\text {out }}$. Thus, according to the XSPARQL BGP matching (cf. Definition 10), $\Omega_{x s}^{\text {in }}$ will contain all the solution mappings that are compatible with any solution mapping $\mu_{i} \in \Omega_{x s}^{\text {out }}$ and specifically those compatible with $\mu_{n l}^{\text {out }}$. Since we know that $\mu_{n l}^{i n}$ is compatible with $\mu_{n l}^{\text {out }}$, we have that $\mu_{n l}^{\text {in }}$ must belong to $\Omega_{x s}^{\text {in }}$; thus we can deduce that dynEnv $\vdash Q \Rightarrow$ Val.

Inductive Step The proof follows from the recursive application of the base case, over a new dynamic environment determined by the $o p t_{n l}$ rewriting to dynEnv ${ }_{i} \vdash$ opt $_{n l}($ ExprSingle).

The proof for nested queries with an XQuery for outer expression (Q2) is analogous where, in the preceding, the evaluation of the SparqlForClause from lines (1)-(3) of (Q3) is replaced by the evaluation of an XQuery ForClause, as presented by Draper et al. [27, Section 4.8.2].

\section{C.7 Proof for Proposition 5}

Proposition 5 Let $Q$ an XSPARQL expression of form (Q4) and dynEnv the dynamic environment of $Q$, then dynEnv $\vdash$ $Q \Rightarrow$ Val if and only if dynEnv $\vdash$ opt $_{s r}(Q) \Rightarrow$ Val.

Proof We start by showing the proof for the base case, where ExprSingle of (Q4) does not contain any occurrences of (Q4).

Base Case. $(\Rightarrow)$ We start by showing that if dynEnv $\vdash$ $Q \Rightarrow$ Val then dynEnv $\vdash$ opt $_{s r}(Q) \Rightarrow$ Val. Consider $\Omega_{x s}^{\text {out }}$ and $\Omega_{x s}^{\text {in }}$ the solution sequences returned, respectively, by the evaluation of the outer and inner SparqlForClauses of $Q$ and $J=\operatorname{Vars}^{\text {out }} \cap \operatorname{Vars}\left(G G P^{\text {in }}\right)$ the set of join variables. Furthermore, consider dynEnv ${ }_{i}^{\text {expr }}$ the dynamic environment resulting from extending dynEnv with the variable mappings from 
the compatible solution mappings $\mu_{x s}^{\text {out }} \in \Omega_{x s}^{\text {out }}$ and $\mu_{x s}^{\text {in }} \in \Omega_{x s}^{\text {in }}$ such that dynEnve ${ }_{i}^{\text {expr }} \vdash$ ExprSingle $\Rightarrow$ Val.

We now show the proof tree for each of the XQuery core expressions in each line of the $o p t_{s r}$ rewriting where, for each line, Expr represents the expressions of the following lines:

- let expression of line (1)

$$
\begin{aligned}
& \text { dynEnv } \vdash \text { xsp:sparqlCal1 }\left(\begin{array}{l}
\text { select Vars }{ }^{\text {out }} \cup \text { Vars }^{\text {in }} \\
\text { DatasetClause } \\
\text { where GGP } G G P^{\text {in }} \\
\text { order by } \text { OC }^{\text {out }} O C^{\text {in }}
\end{array}\right) \\
& \Rightarrow \Omega_{\text {sr }} \\
& \mathrm{dynEnv}_{1}^{s r} \vdash \operatorname{Exp} r \Rightarrow \text { Res } \\
& \text { let \$xsp:results := } \\
& \text { dynEnv } \vdash \quad \text { xsp:sparqlCal1 }\left(\begin{array}{l}
\text { select } \text { Vars }^{\text {out }} \cup \text { Vars }^{\text {in }} \\
\text { DatasetClause } \\
\text { where GGP } G G P^{\text {in }} \\
\text { order by } \text { OC }^{\text {out }} \text { OC } C^{\text {in }}
\end{array}\right)
\end{aligned}
$$

where

$$
\operatorname{dynEnv}_{1}^{s r}=\text { dynEnv }+\operatorname{varValue}\left(\text { xsp:results } \Rightarrow \Omega_{s r}\right) .
$$

According to the SPARQL semantics, the solution sequence that results from evaluating the graph pattern $G G P^{\text {out }} G G P^{\text {in }}, \Omega_{s r}=\Omega_{s r}^{\text {out }} \bowtie \Omega_{s r}^{\text {in }}$ consists of all the solution mappings $\mu_{s r}^{\text {out }} \in \Omega_{s r}^{\text {out }}$ and $\mu_{s r}^{\text {in }} \in \Omega_{s r}^{\text {in }}$ such that $\mu_{s r}^{\text {out }}$ and $\mu_{s r}^{\text {in }}$ are compatible.

The following for expression iterates over all these compatible solution mappings:

- for expression of line (2)

$$
\begin{aligned}
& \text { dynEnv }_{1}^{s r} \vdash \text { \$xsp:results//sr:result } \Rightarrow \mu_{i}
\end{aligned}
$$

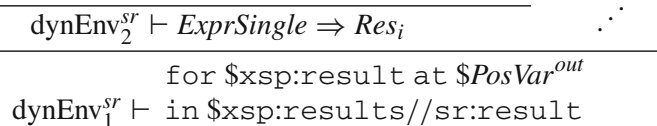

$$
\begin{aligned}
& \text { return ExprSingle } \Rightarrow \operatorname{Res}_{1}, \cdots, \text { Res }_{n}
\end{aligned}
$$

where $\operatorname{dynEnv}_{2}^{s r}=\operatorname{dynEnv}_{1}^{s r}+\operatorname{varValue}\left(\begin{array}{l}\text { xsp:result } \Rightarrow \mu_{i} ; \\ \text { PosVar }{ }^{\text {out }} \Rightarrow i\end{array}\right)$

- let expressions of lines (3)-(4)

Here we consider all the let expressions represented by line (3), where $\$ v \in$ Vars:

$$
\begin{aligned}
& \text { dynEnv }_{2}^{s r} \vdash \$ x \text { sp:result/sr:binding }[@ \text { name }=\$ v] / * \Rightarrow V \\
& \begin{array}{c}
\hline \operatorname{dynEnv}_{3}^{\text {sr }} \vdash \text { ExprSingle } \Rightarrow \text { Res } \\
\qquad \begin{array}{c}
\text { let } \$ v:= \\
\text { dynEnv }{ }_{2}^{\text {sr }} \vdash \text { xisult/sr:binding }[@ \text { name }=v] / * \\
\text { return ExprSingle } \Rightarrow \text { Res }
\end{array} \\
\text { where }
\end{array} \\
& \operatorname{dynEnv}_{3}^{s r}=\operatorname{dynEnv}_{2}^{s r}+\operatorname{varValue}(v \Rightarrow V) .
\end{aligned}
$$

Note that we are only considering order by solution modifiers; thus the number of results of each query is not changed. At most the ordering of the results is changed but this does not interfere with this proof and solution modifiers can be safely ignored in what follows.

Regarding the evaluation of the SparqlForClause from lines (1)-(4) of $Q$ (evaluated considering dynEnv), the $o p t_{s r}(Q)$ translates it into the xsp : sparqlCall from line (1), which is also evaluated over dynEnv. In this case, according to Lemma 2, we have that $\Omega_{s r}^{\text {out }}=\Omega_{x s}^{\text {out }}$ and then $\mu_{x s}^{\text {out }} \in \Omega_{\text {sr }}^{\text {out }}$.

Regarding the evaluation of the SparqlForClause from lines (5)-(8) of $Q$ (evaluated considering some dynamic environment dynEnv $\left.{ }^{e x p r}\right)$, the $\operatorname{opt}_{s r}(Q)$ rewriting incorporates it into the xsp:sparqlCall from line (1), which is also evaluated over dynEnv. Considering that dynEnv is less restrictive than dynEnv ${ }^{\text {expr }}$, i.e., dynEnv contains less bindings for variables than dynEnvexpr, and thus the evaluation of the inner SparqlForClause over dynEnv will contain all the solution mappings from $\Omega_{x s}^{\text {in }}$ and specifically $\mu_{x s}^{\text {in }}$. As $\mu_{x s}^{\text {out }}$ and $\mu_{x s}^{i n}$ are compatible we have that dynEnv $\vdash o p t_{s r}(Q) \Rightarrow$ Val.

$(\Leftarrow)$ Next we show that if dynEnv $\vdash o p t_{s r}(Q) \Rightarrow$ Val then dynEnv $\vdash Q \Rightarrow \mathrm{Val}$. Consider $\Omega_{s r}^{\text {out }}$ and $\Omega_{s r}^{\text {in }}$ as per the $(\Rightarrow)$ direction of the proof and the set of join variables $J=$ Vars $^{\text {out }} \cap \operatorname{vars}\left(G G P^{\text {in }}\right)$. As we have seen $\Omega_{s r}$ contains all the solution mappings $\mu=\mu_{s r}^{\text {out }} \bowtie \mu_{s r}^{\text {in }}$ such that $\mu_{s r}^{\text {out }} \in \Omega_{s r}^{\text {out }}$ and $\mu_{s r}^{\text {in }} \in \Omega_{s r}^{\text {in }}$ and $\mu_{s r}^{\text {out }}$, and $\mu_{s r}^{i n}$ are compatible. Without loss of generality consider $\mu_{s r}^{o u t}$ and $\mu_{s r}^{i n}$ the solution mappings where Val is deduced from.

Let us turn to the evaluation of dynEnv $\vdash Q \Rightarrow$ Val.

- SparqlForClause from lines (1)-(4), where Expr corresponds to the SparqlForClause from lines (5)-(8) of $Q$. Again, the evaluation of this SparqlForClause consists of the application of Rule (D1):

$$
\begin{aligned}
& \text { dynEnv } \vdash f \text { s:dataset(DatasetClause }) \Rightarrow D S \\
& \text { dynEnv } \vdash f \text { s:sparql }\left(\begin{array}{l}
D S \text {, where } G G P^{\text {out }} \\
\text { order by OC }
\end{array}\right) \Rightarrow \mu_{i}
\end{aligned}
$$

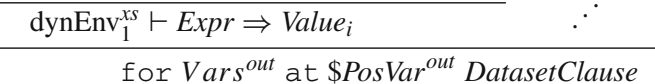

$$
\begin{aligned}
& \text { dynEnv } \vdash \begin{array}{l}
\text { where } G G P^{\text {out }} \\
\text { order by } O C^{\text {out }}
\end{array} \\
& \text { return Expr } \Rightarrow \text { Value }_{1} \cdots \text { Value }_{m}
\end{aligned}
$$

where $\operatorname{Vars}^{\text {out }}=\$ \operatorname{Var}_{1}^{\text {out }} \cdots \$ \operatorname{Var}_{n}^{\text {out }}$, we have for each $\mu_{i}$

$$
\begin{gathered}
\text { dynEnv }+\operatorname{activeDataset}(D S) \\
\operatorname{dynEnv}_{1}^{x s}=+\operatorname{varValue}\left(\begin{array}{l}
\text { PosVar }{ }^{\text {out }} \Rightarrow i \\
\operatorname{Var}_{1}^{\text {out }} \Rightarrow f s: \operatorname{value}\left(\mu_{i}, \operatorname{Var}_{1}^{\text {out }}\right) \\
\cdots ; \\
\operatorname{Var}_{n}^{\text {out }} \Rightarrow f \text { s:value }\left(\mu_{i}, \operatorname{Var}_{n}^{\text {out }}\right)
\end{array}\right)
\end{gathered}
$$


- SparqlForClause of lines (4)-(6):

The evaluation of dynEnv ${ }_{i}^{x s} \vdash$ ExprSingle $^{\text {out }} \Rightarrow$ Value $_{i}$ is shown next:

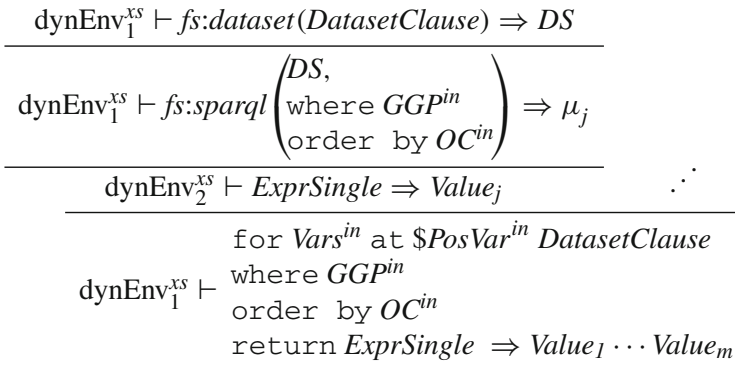

where $\operatorname{Vars}^{i n}=\$ \operatorname{Var}_{1}^{i n} \cdots \$ \operatorname{Var}_{n}^{i n}$, we have for each $\mu_{j}$

$$
\begin{gathered}
\operatorname{dynEnv}_{1}^{x s}+\operatorname{activeDataset}(D S) \\
\operatorname{dynEnv}_{2}^{x s}=+\operatorname{varValue}\left(\begin{array}{l}
\operatorname{Pos} V a r^{i n} \Rightarrow j \\
\operatorname{Var}_{1}^{i n} \Rightarrow f_{s: \operatorname{value}}\left(\mu_{j}, \operatorname{Var}_{l}^{i n}\right) ; \\
\cdots ; \\
\operatorname{Var}{ }_{n}^{i n} \Rightarrow f s: \operatorname{value}\left(\mu_{j}, \operatorname{Var}_{n}^{i n}\right)
\end{array}\right)
\end{gathered}
$$

As we have seen in the $(\Rightarrow)$ direction, we have that $\Omega_{s r}^{\text {out }}=$ $\Omega_{x s}^{\text {out }}$ and so we have that $\mu_{s r}^{\text {out }} \in \Omega_{x s}^{\text {out }}$.

Consider $C$ the expression context where dynEnv is included and $\mu_{C}$ the XSPARQL instance mapping of $C$. Further, consider $P^{i n}$ the graph pattern obtained from replacing the variables in $G G P^{i n}$ according to $\mu_{C}$. Since $\operatorname{vars}\left(G G P^{i n}\right) \subseteq \operatorname{vars}\left(P^{i n}\right)$ all solutions mappings returned by evaluating $G G P^{i n}$ under XSPARQL semantics are included in the solution sequence of evaluating $P^{i n}$ under SPARQL semantics, i.e., $\Omega_{x s}^{i n} \preceq \Omega_{s r}^{i n}$. We obtain two cases: (i) $\mu_{s r}^{i n} \in \Omega_{x s}^{i n}$ or (ii) $\mu_{s r}^{i n} \notin \Omega_{x s}^{i n}$. In (i) we immediately get that dynEnv $\vdash Q \Rightarrow V a l$. For (ii), consider $\mu_{C_{I}}^{x s}$ the XSPARQL instance of the inner SparqlForClause (created based on dynEnv ${ }_{1}^{x s}$ ). As we can see from (T5), dynEnv $_{1}^{x s}$ (and thus also $\mu_{C_{l}}^{x s}$ ) includes the bindings for variables from each solution mapping $\mu_{i} \in \Omega_{x s}^{\text {out }}$. Thus, according to the XSPARQL BGP matching (cf. Definition 10), $\Omega_{x s}^{i n}$ will contain all the solution mappings that are compatible with any solution mapping $\mu_{i} \in \Omega_{x s}^{\text {out }}$ and specifically those compatible with $\mu_{s r}^{\text {out }}$. Since we know that $\mu_{s r}^{i n}$ is compatible with $\mu_{s r}^{\text {out }}$, we have that $\mu_{s r}^{i n}$ must belong to $\Omega_{x s}^{i n}$; thus we can deduce that dynEnv $\vdash Q \Rightarrow$ Val.

Inductive Step The proof follows from the recursive application of the base case, over a new dynamic environment determined by the $o p t_{s r}$ rewriting to dynEnv ${ }_{i} \vdash$ opt $_{\text {sr }}($ ExprSingle $)$.

\section{C.8 Proof for Proposition 6}

Proposition 6 Let $Q$ be an XSPARQL expression ofform (Q5) and dynEnv the dynamic environment of $Q$, then dynEnv $\vdash$ $Q \Rightarrow$ Val if and only if dynEnv $\vdash$ opt $_{n g}(Q) \Rightarrow$ Val.

Proof We start by showing the proof for the base case, where ExprSingle $_{1}$ and ExprSingle 2 of (Q5) do not contain any occurrences of (Q5).

Base Case. $(\Rightarrow)$ Let us start by showing that if dynEnv $\vdash$ $Q \Rightarrow$ Val then dynEnv $\vdash o p t_{n g}(Q) \Rightarrow$ Val. Consider $\Omega_{x s}^{\text {in }}$ the solution sequence returned by the evaluation of the inner SparqlForClauses of $Q$. Furthermore, consider dynEnv ${ }_{i}^{\text {expr }}$ such that dynEnv $_{i}^{\text {expr }} \vdash$ ExprSingle $_{2} \Rightarrow$ Val. The dynamic environment dynEnv ${ }_{i}^{\text {expr }}$ results from extending dynEnv with bindings for the outer variable \$VarName and with the variable bindings from a solution mapping $\mu_{x s}^{i n} \in \Omega_{x s}^{i n}$ where $\mu_{x s}^{i n}($ VarName $)=\$$ VarName, i.e., the value for the join variable in the solution mapping $\mu_{x s}^{i n}$ is the same as assigned to \$VarName.

We now show the proof tree for each of the XQuery core expressions in the $o p t_{n g}$ rewriting.

- let expression of line (1)

Considering $N G P=\{[]$ :value $\$$ VarName $\}$, we have

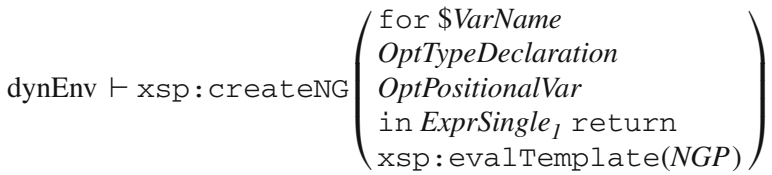

$$
\begin{aligned}
& \Rightarrow D S \\
& \text { dynEnv }_{1}^{n g} \vdash \text { ExprSingle }_{2} \Rightarrow \text { Res } \\
& \text { let } \$ x \text { sp:ds := } \\
& \operatorname{dynEnv} \vdash \quad \mathrm{xsp}: \text { createNG }\left(\begin{array}{l}
\text { for } \$ \text { WarName } \\
\text { OptTypeDeclaration } \\
\text { OptPositionalVar } \\
\text { in ExprSingle } \text { Eeturn }_{1} \text { retate }(N G P)
\end{array}\right) \\
& \text { return ExprSingle } \Rightarrow \text { Res }
\end{aligned}
$$

where

$$
\operatorname{dynEnv}_{1}^{n g}=\operatorname{dynEnv}+\operatorname{varValue}(x \mathrm{sp}: \mathrm{ds} \Rightarrow D S) .
$$

- let expression of line (2)

Consider the dataset clause DatasetClause ${ }^{\text {ng }}=$ DatasetClause $\cup$ from named \$xsp:ds\} and the graph pattern WhereClause ${ }^{n g}=$ WhereClause $\cup$ where $\{g r a p h$ \$xsp:ds $\{[]$ :value \$VarName $\}$. 


$$
\begin{aligned}
& \operatorname{dynEnv}_{1}^{n g} \vdash \mathrm{xsp}: \text { sparq1Cal1 }\left(\begin{array}{l}
\text { select } \\
\text { Vars } \cup\{\text { \$VarName }\} \\
\text { DatasetClause } \\
\text { WhereClause }^{n g} \\
\text { SolutionModifier }
\end{array}\right) \Rightarrow \Omega \\
& \text { dynEnv }_{2}^{n g} \vdash \text { ExprSingle }_{2} \Rightarrow \text { Res } \\
& \text { let \$xsp:results := }
\end{aligned}
$$

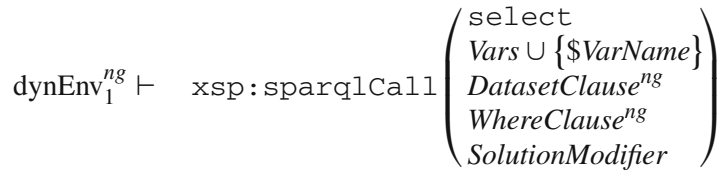

$$
\begin{aligned}
& \text { return ExprSingle } 2 \Rightarrow \text { Res }
\end{aligned}
$$

where

$$
\text { dynEnv }_{2}^{n g}=\text { dynEnv }_{1}^{n g}+\operatorname{varValue}(\text { xsp:results } \Rightarrow \Omega)
$$

The new merged dataset, DatasetClause ${ }^{n g}$, is created based on DatasetClause and the newly created named graph $N G$. Since the URI that identifies the newly created named graph $N G$ is distinct from any URI of named graphs present in DatasetClause, the triples included in $N G$ will never be a solution for WhereClause, and will be matched only by the graph pattern where $\{$ graph \$xsp:ds $\{[]$ :value \$VarName $\}\}$.

Consider $C$ the expression context where dynEnv is included, $\mu_{C}$ the XSPARQL instance mapping of $C$ and $P^{\text {out }}$ and $P^{\text {in }}$ the graph patterns obtained from, respectively, replacing the variables in WhereClause and where $\{$ graph \$xsp:ds $\{[]$ :value \$VarName $\}\}$ according to $\mu_{C}$.

Furthermore, let $\Omega_{n g}^{\text {out }}=\operatorname{eval}\left(\right.$ DatasetClause $\left.{ }^{\text {ng }}, P^{\text {out }}\right)$ and $\Omega_{n g}^{i n}=\operatorname{eval}\left(\right.$ DatasetClause $\left.e^{n g}, P^{i n}\right)$. According to SPARQL semantics, the pattern solution that results from evaluating WhereClause, $\Omega_{n g}=\Omega_{n g}^{\text {out }} \bowtie \Omega_{n g}^{\text {in }}$ consists of all the solution mappings $\mu_{\text {out }} \in \Omega_{n g}^{\text {out }}$ and $\mu_{\text {in }} \in \Omega_{n g}^{\text {in }}$ such that $\mu_{\text {out }}$ and $\mu_{\text {in }}$ are compatible.

- for expression of line (3)

$$
\begin{gathered}
\frac{\text { dynEnv }_{2}^{n g} \vdash \text { \$xsp:results//sr:result } \Rightarrow \mu_{i}}{\text { dynEnv }_{3}^{n g} \vdash \text { ExprSingle }_{2} \Rightarrow \operatorname{Res}_{i}} \\
\text { dynEnv }_{2}^{n g} \vdash \quad \text { for \$xsp:result at \$xsp:result_pos } \\
\text { in \$xsp:results//sr:result } \\
\text { return ExprSingle } \Rightarrow \text { Res }_{1}, \cdots, \text { Res }_{n}
\end{gathered}
$$

where

$$
\operatorname{dynEnv}_{3}^{n g}=\operatorname{dynEnv}_{2}^{n g}+\operatorname{varValue}\left(\begin{array}{l}
\text { xsp:result } \Rightarrow \mu_{i} ; \\
\text { xsp:result_pos } \Rightarrow i
\end{array}\right) .
$$

- let expressions of lines (4)-(5)

Here we consider all the let expressions represented by line (4), where $\$ v \in$ Vars:

$$
\frac{\text { dynEnv }_{3}^{n g} \vdash \text { Xxsp:result/sr:binding }[@ \text { name }=\$ v] / * \Rightarrow V}{\text { dynEnv }_{4}^{n g} \vdash \text { ExprSingle }_{2} \Rightarrow \operatorname{Res}}
$$

where

$$
\text { dynEnv }_{4}^{n g}=\text { dynEnv }_{3}^{n g}+\operatorname{varValue}(v \Rightarrow V) .
$$

Similarly to the proof of Proposition 5, we are only considering order by solution modifiers; these only change the order of the solution sequences and thus can be safely ignored for this proof.

Regarding the evaluation of the XQuery for clause from lines (1)-(2) of $Q$ (evaluated considering dynEnv), the $o p t_{n g}(Q)$ translates it into the x.sp : sparqlCall from line (2), which is evaluated considering dynEnv ${ }_{1}^{n g}$. As we can see from (T6), dynEnv ${ }_{1}^{n g}$ is based on dynEnv by adding the binding for the xsp:ds variable. Since this variable belongs to the xsp: reserved namespace, it is not allowed in the WhereClause and so we have that the results of evaluating the $\mathrm{xsp}$ : sparqlCall function over dynEnv or dynEnv ${ }_{1}^{n g}$ will be the same.

Regarding the evaluation of the SparqlForClausefrom lines (3)-(4) of $Q$ (evaluated considering some dynamic environment dynEnv ${ }^{\text {expr }}$ ), the $\operatorname{opt}_{n g}(Q)$ also incorporates it into the xsp: sparqlCall from line (2), which is evaluated over dynEnv $_{1}^{n g}$. Considering that dynEnv ${ }_{1}^{n g}$ is less restrictive than dynEnv $^{\text {expr }}$, i.e. dynEnv ${ }_{1}^{n g}$ contains less bindings for variables than dynEnv ${ }^{\text {expr }}$, the evaluation of the inner SparqlForClauseover dynEnv ${ }_{1}^{n g}$ will contain all the solution mappings from $\Omega_{x s}^{i n}$ and specifically $\mu_{i n}$. As $\mu_{\text {out }}$ and $\mu_{\text {in }}$ are compatible we have that dynEnv $\vdash n g($ expr $) \Rightarrow$ Val.

$(\Leftarrow)$ Next we will show that if dynEnv $\vdash o p t_{n g}(Q) \Rightarrow \mathrm{Val}$ then dynEnv $\vdash Q \Rightarrow$ Val. Consider $\Omega_{n g}^{\text {out }}$ and $\Omega_{n g}^{\text {in }}$ the solution sequences returned by, respectively, the evaluation of the new WhereClause $e^{n g}$ and WhereClause. As we have seen $\Omega_{n g}$ contains all the solution mappings $\mu=\mu_{n g}^{\text {out }} \bowtie \mu_{n g}^{\text {in }}$, where $\mu_{n g}^{\text {out }} \in \Omega_{n g}^{\text {out }}$ and $\mu_{n g}^{\text {in }} \in \Omega_{n g}^{\text {in }}$, such that $\mu_{n g}^{\text {out }}$ and $\mu_{n g}^{\text {in }}$ are compatible. Again, consider $\mu_{n g}^{\text {out }}$ and $\mu_{n g}^{\text {in }}$ the pattern solutions where Val is deduced from.

Let us turn to the evaluation of dynEnv $\vdash Q \Rightarrow$ Val.

- XQuery for clause from lines (1)-(2):

Expr corresponds to the SparqlForClause from lines (3)(4) of $Q$. 


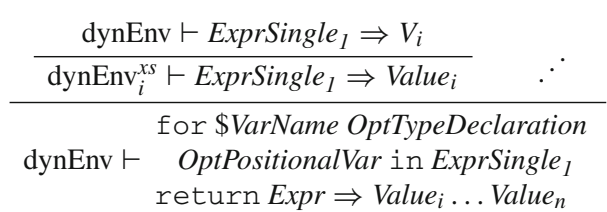

we have for each $V_{i}$ :

$$
\operatorname{dynEnv}_{i}^{x s}=\operatorname{dynEnv}+\operatorname{varValue}\left(\operatorname{VarName} \Rightarrow V_{i}\right) .
$$

- SparqlForClause of lines (2)-(4):

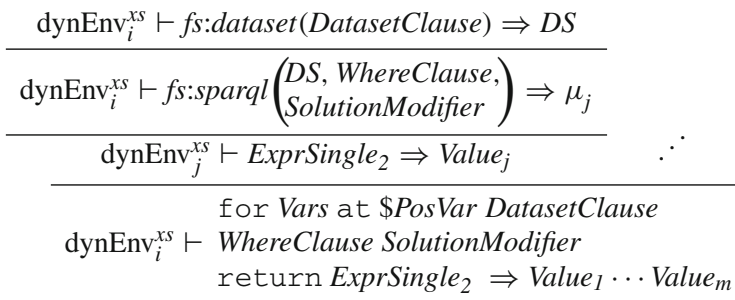

where, considering Vars $=\$ \operatorname{Var}_{1} \ldots \$ \operatorname{Var}_{n}$, we have for each $\mu_{j}$ :

$$
\begin{aligned}
\operatorname{dynEnv}_{i}^{x s}+\operatorname{activeDataset}(D S) \\
\operatorname{dynEn}_{j}^{x s}=+\operatorname{varValue}\left(\begin{array}{l}
\text { PosVar } \Rightarrow j ; \\
\operatorname{Var}_{l} \Rightarrow f \text { s:value }\left(\mu_{j}, \text { Var }_{l}\right) \\
\cdots ; \\
\operatorname{Var}_{n} \Rightarrow f \text { s:value }\left(\mu_{j}, \text { Var }_{n}\right)
\end{array}\right)
\end{aligned}
$$

As we have seen in the $(\Rightarrow)$ direction, we have that $\Omega_{n g}^{\text {out }}=$ $\Omega_{x s}^{\text {out }}$ and so we have that $\mu_{n g}^{\text {out }} \in \Omega_{x s}^{\text {out }}$.

Consider $C$ the expression context where dynEnv is included and $\mu_{C}$ the XSPARQL instance mapping of $C$. Further consider $P^{\text {in }}$ the graph pattern obtained from replacing the variables in WhereClause ${ }^{i n}$ according to $\mu_{C}$. Since we know that vars $\left(\right.$ WhereClause $\left.e^{i n}\right) \subseteq \operatorname{vars}\left(P^{\text {in }}\right)$, all solutions mappings returned by evaluating WhereClause $e^{\text {in }}$ under XSPARQL semantics are included in the pattern solution of evaluating $P^{i n}$ under SPARQL semantics, i.e., $\Omega_{x s}^{i n} \preceq \Omega_{n g}^{i n}$. We obtain two cases: (i) $\mu_{n g}^{i n} \in \Omega_{x s}^{i n}$; or (ii) $\mu_{n g}^{i n} \notin \Omega_{x s}^{i n}$. In (i) we immediately get that dynEnv $\vdash Q \Rightarrow \mathrm{Val}$. For (ii), consider $\mu_{C_{l}}^{x s}$ the XSPARQL instance of the inner SparqlForClause (created based on dynEnv ${ }_{1}^{x S}$ ). As we can see from (T7), $\operatorname{dynEnv}_{1}^{x s}$ (and thus also $\mu_{C_{I}}^{x s}$ ) includes the bindings for variables from each solution mapping $\mu_{i} \in \Omega_{x s}^{\text {out }}$. Thus, according to the XSPARQL BGP matching (cf. Definition 10), $\Omega_{x s}^{\text {in }}$ will contain all the solution mappings that are compatible with any solution mapping $\mu_{i} \in \Omega_{x s}^{\text {out }}$ and specifically those compatible with $\mu_{n g}^{\text {out }}$. Since we know that $\mu_{n g}^{i n}$ is compatible with $\mu_{n g}^{\text {out }}$, we have that $\mu_{n g}^{i n}$ must belong to $\Omega_{x s}^{i n}$; thus we can deduce that dynEnv $\vdash Q \Rightarrow$ Val.
Inductive Step. Let us assume that, for some arbitrary dynEnv $_{i}$, dynEnv $_{i} \vdash$ ExprSingle $_{1} \Rightarrow \mathrm{Val}_{i}$ if and only if $\operatorname{dynEnv}_{i} \vdash \operatorname{opt}_{n g}\left(\right.$ ExprSingle $\left._{1}\right) \Rightarrow V a_{i}$. According to the opt $_{n g}$ rewriting, there must exist a dynEnv ${ }_{j}$ that is the extension of dynEnv ${ }_{i}$ with $V_{a l}$ and thus dynEnv ${ }_{j} \vdash$ ExprSingle $_{2} \Rightarrow$ Val if and only if dynEnv ${ }_{j} \vdash$ opt $_{n g}\left(\right.$ ExprSingle $\left._{2}\right)$ $\Rightarrow \mathrm{Val}$. Consequently, we have that dynEnv $\vdash Q \Rightarrow \mathrm{Val}$ if and only if dynEnv $\vdash o p t_{n g}(Q) \Rightarrow$ Val .

\section{The XMarkRDF Benchmark}

For the evaluation of our implementation we created a benchmark suite based on the XMark benchmark suite [57]. According to [3], the XMark suite is the most widely used benchmark suite for XQuery. It provides a data generator that produces XML data simulating an auction website (including information about persons and items they bid for) and includes $20 \mathrm{XQuery}$ queries, referred to as $q_{1}$ to $q_{20}$ henceforth, over this generated data.

In order to benchmark the XSPARQL language we also require data in RDF format; hence we provide transformations (in fact, using XSPARQL queries) from XML datasets generated by XMark into RDF triples. In this transformation we replicate all the data in the original XMark datasets as RDF triples. We start by generating IRIs for each XML element that represents concepts like "persons," "items," "bids," etc. Inner XML element names are then converted into RDF predicates and used to link the generated IRIs to the leaf element values which are converted into RDF literals. Next, we converted the XMark queries into corresponding XSPARQL queries using SparqlForClauses to access the RDF data. We call this new benchmark suite the XMarkRDF benchmark and is available for download at http://xsparql.deri.org/data/ $\mathrm{XMarkRDF} /$.

From the initial set of 20 queries there are 5 queries $\left(q_{8}-\right.$ $q_{12}$ ) which contain nested expressions. They are described informally in the XMark suite as follows:

(q8) "List the names of persons and the number of items they bought;"

(q9) "List the names of persons and the names of the items they bought in Europe;"

$\left(q_{10}\right)$ "List all persons according to their interest;"

$\left(q_{11}\right)$ "List the number of items currently on sale whose price does not exceed $0.02 \%$ of the seller's income;" and

$\left(q_{12}\right)$ "For each richer-than-average person, list the number of items currently on sale whose price does not exceed $0.02 \%$ of the person's income."

Figure $20 \mathrm{a}, \mathrm{b}$ presents XMark query $q_{9}$ and its translated XSPARQL version in XMarkRDF, respectively. We have made two changes to the XMark queries: (1) SPARQL que- 


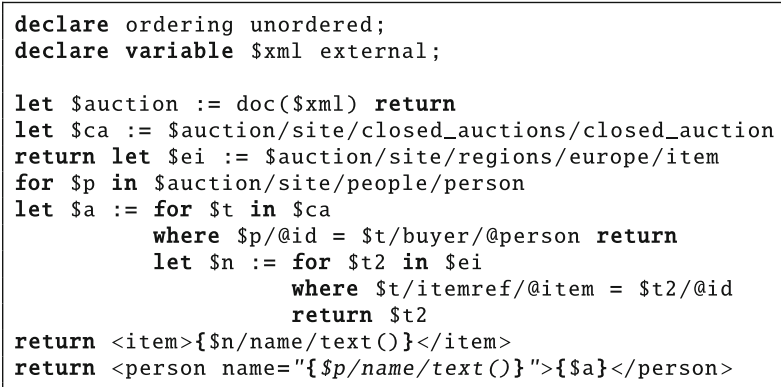

(a) Query $q_{9}$ in XQuery (XMark)

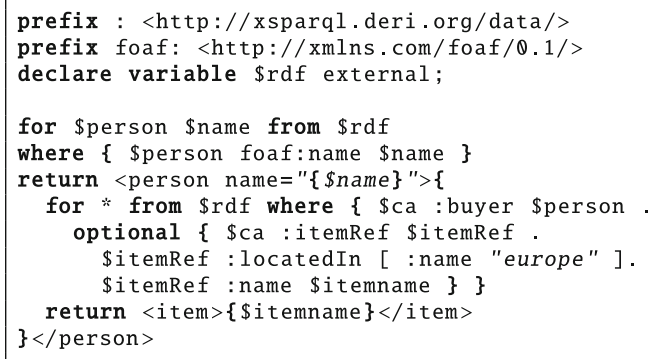

(b) Query $q_{9}$ in XSPARQL (XMarkRDF)

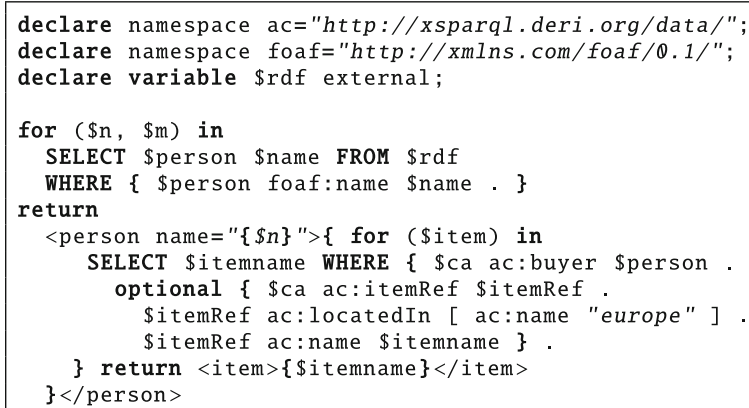

(c) Query $q_{9}$ in SPARQL2XQuery $\left(\mathrm{XMarkRDF}_{S 2 X Q}\right)$

Fig. 20 Variants of benchmark query $q_{9}$

ries do not guarantee any default ordering; hence all original XMark queries were declared unordered-as a consequence the XQuery engine is not required to follow document order when executing the query; and (2) we added the external variables $\$ x m l$ and $\$ r d f$ in the XQuery and XSPARQL query, respectively, as parameters used to specify the URL identifying the input benchmark instance.

We included the SPARQL2XQuery system, which is similar in spirit to XSPARQL, by [33] in our system comparison. While the language allows to perform similar queries to the XSPARQL language, the implementation follows a different approach to integrate the XML and RDF data. Rather than performing interleaved calls to a SPARQL engine, the SPARQL2XQuery system relies on translating the RDF data into a pre-defined XML format and transforming SPARQL queries into equivalent XQuery over the pre-defined XML format. The translated queries can be directly executed using
Table $4 \mathrm{XMarkRDF}_{S 2 X O}$ dataset and translation times

\begin{tabular}{lcc}
\hline $\begin{array}{l}\text { Scaling } \\
\text { factor }\end{array}$ & $\begin{array}{l}\text { Dataset } \\
\text { size (MB) }\end{array}$ & $\begin{array}{l}\text { Translation } \\
\text { times (s) }\end{array}$ \\
\hline 0.01 & 3.3 & 18.94 \\
0.02 & 6.4 & 18.30 \\
0.05 & 16.1 & 26.08 \\
0.10 & 32.7 & 39.01 \\
0.20 & 65.3 & 62.35 \\
0.50 & 162.3 & 143.35 \\
1.00 & 326.2 & 329.93 \\
\hline
\end{tabular}

a native XQuery engine. For further comparison between XSPARQL and the SPARQL2XQuery language, and other related works, we refer the reader to Sect. 8.

Query $q_{9}$, as presented in Fig. 20c, is ready to be evaluated by the SPARQL2XQuery system over the XMarkRDF S2XQ $_{2}$ dataset. Please note that this query follows the syntax presented in [33], since we only had access to the implementation of the translation from SPARQL to XQuery, while the evaluation was done using the associated XQuery code. We focussed in our experimental evaluation on query response time rather than on data transformation time, and as SPARQL2XQuery requires an additional translation step from RDF to a custom RDF/XML format, we converted the XMarkRDF RDF data into the format required by the SPARQL2XQuery system. An overview of this translation process, including the translation times, is presented in Table 4. We denote these new datasets, containing the RDF/XML format required for the SPARQL2XQuery, by $\mathrm{XMarkRDF}_{S 2 \mathrm{XQ}}$.

\section{Optimising XMarkRDF Nested Queries}

The different rewritings presented in Sect. 6 can be applied to the four nested queries $q_{8}-q_{11}$. Query $q_{12}$ also consists of a nested expression; however, the most accurate translation of this query into XSPARQL results in the dependent variable not being strictly bound since it occurs only in the filter of the inner query. As such, we cannot apply the different rewritings to this query.

XMarkRDF query $q_{9}$ is presented in Fig. 20 on the facing page. This query is close to queries $q_{8}, q_{10}$, and $q_{11}$ and consists of a nested expression: the inner for expression of the query (lines 8-12) is executed once for each person matched by the outer expression (lines 5-6), which means that one SPARQL call will be made for each person separately. Thus, the number of SPARQL calls performed in the inner expression directly depends on the size of the dataset (cf. Table 5 for details). Queries $q_{8}, q_{9}$, and $q_{11}$ evaluate the inner expression for each person, while $q_{10}$ evaluates the inner expression for each category. Each dataset contains usually about 25 times 
Table 5 Benchmark dataset description

\begin{tabular}{lrccc}
\hline $\begin{array}{l}\text { Scaling } \\
\text { factor }\end{array}$ & Persons & Categories & XMark (MB) & XMarkRDF (MB) \\
\hline 0.01 & 255 & 10 & 1.1 & 1.2 \\
0.02 & 510 & 20 & 2.3 & 2.3 \\
0.05 & 1,275 & 50 & 5.8 & 5.8 \\
0.10 & 2,550 & 100 & 11.7 & 12.4 \\
0.20 & 5,100 & 200 & 23.5 & 24.9 \\
0.50 & 12,750 & 500 & 58.0 & 61.7 \\
1.00 & 25,500 & 1,000 & 116.5 & 124.8 \\
\hline
\end{tabular}

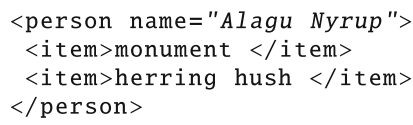

(a) Query $q_{9}$ - bought items grouped by person

$<$ item name="monument " $>$ Alagu Nyrup $</$ item $>$ $<$ item name="herring hush " $>$ Alagu Nyrup $</$ item $>$

(b) Query $q_{9}^{\prime}$ - flat list of items and buyer

Fig. 21 Example output excerpts of queries $q_{9}$ and $q_{9}^{\prime}$

more persons than categories. The rewriting strategies presented in Sect. 6 reduce the number of SPARQL calls to two: one to get all the people (similar to the direct rewriting version), and one additional SPARQL call for retrieving all the information about all the auctions in the dataset. Although the query remains exponential, the practical evaluation will show that reducing the number of SPARQL calls drastically improves query execution times (Table 2).

As mentioned in Sect. 6.2, for the SPARQL-based rewritings, we want the query output to be computable directly in SPARQL without any further processing, i.e., we do not want to use XQuery for further processing of the SPARQL results, and the query should be expressible in SPARQL without features from SPARQL 1.1. Since the original nested queries $q_{8}-q_{11}$ group the output results (while optionally applying some aggregation function), we need to include modified versions of these benchmark queries for the evaluation of the SPARQL based rewritings. In these modified queries, denoted $q_{8}^{\prime}-q_{11}^{\prime}$, we changed the return format of the queries to consist of a flattened representation of the output of the original query. An example of the output for queries $q_{9}$ and $q_{9}^{\prime}$ is presented in Fig. 21. All queries $q_{i}^{\prime}$ and $q_{i}^{\prime \prime}$ follow a similar strategy for reformatting the output: the queries resulting from applying opt $t_{s r}$ are named $q_{8}^{\prime}-q_{11}^{\prime}$, while the queries that consist of an outer for expression-to which $o p t_{n g}$ was applied-are $q_{8}^{\prime \prime}-q_{11}^{\prime \prime}$.

\section{References}

1. Abiteboul S, Hull R, Vianu V (1995) Foundations of databases. Addison-Wesley, New York

2. Adida B, Birbeck M, McCarron S, Pemberton S (2008) RDFa in XHTML: syntax and processing. W3C recommendation, W3C. http://www.w3.org/TR/2008/REC-rdfa-syntax-20081014

3. Afanasiev L, Marx M (2008) An analysis of XQuery benchmarks. Inf Syst 33(2):155-181

4. Akhtar W, Kopecký J, Krennwallner T, Polleres A (2008) XSPARQL: traveling between the XML and RDF worlds - and avoiding the XSLT pilgrimage. In: ESWC'08. Springer, Berlin, pp 432-447

5. Angles R, Gutierrez C (2010) SQL nested queries in SPARQL. In: AMW'10, CEUR-WS.org, vol 619

6. Battle S (2006) Gloze: XML to RDF and back again. In: Jena user conference'06

7. Beckett D, Berners-Lee T (2008) Turtle-terse RDF triple language. http://www.w3.org/TeamSubmission/turtle/

8. Beckett D, Broekstra J (2008) SPARQL query results XML format. W3C recommendation, W3C. http://www.w3.org/TR/2008/RECrdf-sparql-XMLres-20080115/

9. Beckett D, McBride B (eds) (2004) RDF/XML syntax specification (Revised). W3C recommendation, W3C. http://www.w3.org/ TR/2004/REC-rdf-syntax-grammar-20040210/

10. Berger S, Bry F, Furche T, Linse B, Schroeder A (2006) Beyond XML and RDF: the versatile Web query language Xcerpt. In: Carr L, Roure DD, Iyengar A, Goble CA, Dahlin M (eds) WWW. ACM, New York, pp 1053-1054

11. Berglund A, Boag S, Chamberlin D, Fernández MF, Kay M, Robie J, Siméon J (2010) XML path language (XPath) 2.0, 2nd edn. W3C recommendation, World Wide Web consortium. http://www. w3.org/TR/2010/REC-xpath20-20101214/

12. Berrueta D, Labra JE, Herman I (2008) XSLT+SPARQL: scripting the semantic web with SPARQL embedded into XSLT stylesheets. In: Workshop on scripting for the semantic web

13. Beyer KS, Cochrane R, Josifovski V, Kleewein J, Lapis G, Lohman GM, Lyle R, Özcan F, Pirahesh H, Seemann N, Truong TC, der Linden BV, Vickery B, Zhang C (2005) System RX: one part relational, one part XML. In: Özcan F (ed) SIGMOD conference. ACM, New York, pp 347-358

14. Bikakis N, Gioldasis N, Tsinaraki C, Christodoulakis S (2009) Querying XML Data with SPARQL. In: DEXA'09, vol 5690. Springer, Berlin, pp 372-381

15. Bischof $S$ (2010) Full XSPARQL grammar. http://xsparql.deri.org/ doc/grammar.html

16. Bischof S (2012) Optimising XML-RDF data integration. In: Simperl E (ed) ESWC 2012. LNCS, vol 7295. Springer, Heidelberg, pp 838-843

17. Bohring H, Auer S (2005) Mapping XML to OWL ontologies. In: Leipziger Informatik-Tage, GI, vol 72, pp 147-156

18. Bray T, Paoli J, Sperberg-Mcqueen CM, Maler E, Yergeau F (2008) Extensible markup language (XML) 1.0, 5th edn. W3C recommendation, World Wide Web consortium. http://www.w3.org/TR/ 2008/REC-xml-20081126/

19. Brickley D, Guha R (2004) RDF vocabulary description language 1.0: RDF schema. W3C recommendation, W3C. http://www.w3. org/TR/2004/REC-rdf-schema-20040210/

20. Brickley D, Miller L (2007) FOAF vocabulary specification. http:// xmlns.com/foaf/spec/

21. Carroll JJ, Stickler P (2004) TriX, RDF triples in XML. Tech. Rep. HPL-2003-268, HP Labs. http://www.hpl.hp.com/ techreports/2004/HPL-2004-56.html

22. Chamberlin D, Robie J, Boag S, Fernández MF, Siméon J, Florescu D (2010) XQuery 1.0: an XML query language, 2nd edn. 
W3C recommendation, W3C. http://www.w3.org/TR/2010/RECxquery-20101214/

23. Clark KG, Feigenbaum L, Torres E (2008) SPARQL protocol for RDF. W3C recommendation, W3C. http://www.w3.org/TR/2008/ REC-rdf-sparqlprotocol-20080115/

24. Connolly D (2007) Gleaning resource descriptions from dialects of languages (GRDDL). W3C recommendation, W3C. http://www. w3.org/TR/2007/REC-grddl-20070911/

25. Corby O, Kefi-Khelif L, Cherfi H, Gandon F, Khelif K (2009) Querying the semantic web of data using SPARQL, RDF and XML. Tech. Rep. 6847, Institut National de Recherche en Informatique et en Automatique

26. Deursen DV, Poppe C, Martens G, Mannens E, Walle RVd (2008) XML to RDF conversion: a generic approach. In: AXMEDIS'08. IEEE, pp 138-144

27. Draper D, Fankhauser P, Fernández M, Malhotra A, Rose K, Rys M, Siméon J, Wadler P (2010) XQuery 1.0 and XPath 2.0 formal semantics, 2nd edn. W3C recommendation, W3C. http://www.w3. org/TR/2010/REC-xquerysemantics-20101214/

28. Droop M, Flarer M, Groppe J, Groppe S, Linnemann V, Pinggera J, Santner F, Schier M, Schopf F, Staffler H, Zugal S (2008) Embedding XPath queries into SPARQL queries. In: ICEIS'08, pp 5-14

29. Farrell J, Lausen H (2007) Semantic annotations for WSDL and XML schema. W3C recommendation, W3C. http://www.w3.org/ TR/2007/REC-sawsdl-20070828/

30. Fernández MF, Malhotra A, Marsh J, Nagy M, Walsh N (2010) XQuery 1.0 and XPath 2.0 data model (XDM), 2nd edn. W3C recommendation, W3C. http://www.w3.org/TR/2010/RECxpath-datamodel-20101214/

31. Fischer P, Florescu D, Kaufmann M, Kossmann D (2011) Translating SPARQL and SQL to XQuery. In: XMLPrague'11, pp 81-98

32. Gearon P, Passant A, Polleres A (2011) SPARQL 1.1 update. W3C working draft, W3C. http://www.w3.org/TR/2011/WDsparq111update-20110512/

33. Groppe S, Groppe J, Linnemann V, Kukulenz D, Hoeller N, Reinke C (2008) Embedding SPARQL into XQuery/XSLT. In: SAC'08. ACM, New York, pp 2271-2278

34. Grust T, Sakr S, Teubner J (2004) XQuery on SQL hosts. In: Nascimento MA, Özsu MT, Kossmann D, Miller RJ, Blakeley JA, Schiefer KB (eds) VLDB. Morgan Kaufmann, pp 252-263

35. Grust T, Rittinger J, Teubner J (2007) eXrQuy: order indifference in XQuery. In: Chirkova R, Dogac A, Özsu MT, Sellis TK (eds) ICDE. IEEE, pp 226-235

36. Grust T, Mayr M, Rittinger J (2010) Let SQL drive the XQuery workhorse (XQuery join graph isolation). In: EDBT'10, vol 426. ACM, pp 147-158

37. Hartig O, Heese R (2007) The SPARQL query graph model for query optimization. In: Franconi E, Kifer M, May W (eds) ESWC, vol 4519. Springer, Berlin, pp 564-578

38. Harris S, Seaborne A (2011) SPARQL 1.1 query language. W3C working draft, W3C. http://www.w3.org/TR/2011/WDsparql11query-20110512/

39. Hayes P (2004) RDF semantics. W3C recommendation, W3C. http://www.w3.org/TR/2004/REC-rdf-mt-20040210/
40. Iannella R (2010) Representing vCard Objects in RDF. http://www. w3.org/Submission/vcard-rdf/. W3C member submission

41. Katz H, Chamberlin D, Kay M, Wadler P, Draper D (2003) XQuery from the experts: a guide to the W3C XML query language. Addison-Wesley

42. Kay M (ed) (2007) XSL transformations (XSLT) version 2.0. W3C recommendation, W3C. http://http://www.w3.org/TR/2007/RECxslt20-20070123/

43. Koch C (2006) On the complexity of nonrecursive XQuery and functional query languages on complex values. ACM Trans Database Syst 31(4):1215-1256

44. Kopecký J, Vitvar T, Bournez C, Farrell J (2007) SAWSDL: semantic annotations for WSDL and XML schema. IEEE Internet Comput 11(6):60-67

45. Malhotra A, Melton J, Walsh N (eds) (2010) XQuery 1.0 and XPath 2.0 functions and operators, 2 nd edn. W3C recommendation, W3C. http://www.w3.org/TR/2010/REC-xpath-functions-20101214/

46. Manola F, Miller E (2004) RDF primer. W3C recommendation, W3C. http://www.w3.org/TR/2004/REC-rdf-primer-20040210/

47. May N, Helmer S, Moerkotte G (2003) Three cases for query decorrelation in XQuery. In: Xsym'03, vol 2824. Springer, Berlin, pp 70-84

48. May N, Stuckenschmidt H (2007) Querying embedded RDF with XML technology: a feasibility study. In: XML Tage 2007. Freie University, Berlin

49. Passant A, Kopecký J, Corlosquet S, Berrueta D, Palmisano D, Polleres A (2009) XSPARQL: use cases. http://www.w3.org/ Submission/xsparql-use-cases/. W3C member submission

50. Pérez J, Arenas M, Gutierrez C (2008) nSPARQL: a navigational language for RDF. In: ISWC'08, vol 5318. Springer, Berlin, pp 66-81

51. Pérez J, Arenas M, Gutierrez C (2009) Semantics and complexity of SPARQL. ACM Trans Database Syst 34(3):1-45

52. Polleres A (2007) From SPARQL to rules (and back). In: WWW'07

53. Polleres A, Scharffe F, Schindlauer R (2007) SPARQL++ for mapping between RDF vocabularies. In: ODBASE'07. Springer, Berlin

54. Prud'hommeaux E, Seaborne A (eds) (2008) SPARQL query language for RDF. W3C recommendation, W3C. http://www.w3.org/ TR/2008/REC-rdf-sparql-query-20080115/

55. Robie J, Chamberlin D, Dyck M, Florescu D, Melton J, Siméon J (2011) XQuery update facility 1.0. W3C recommendation, W3C. http://www.w3.org/TR/xquery-update-10/

56. Rodrigues T, Rosa P, Cardoso J (2008) Moving from syntactic to semantic organizations using JXML2OWL. Comput Ind 59(8):808-819

57. Schmidt A, Waas F, Kersten ML, Carey MJ, Manolescu I, Busse R (2002) XMark: a benchmark for XML data management. In: VLDB'02. Morgan Kaufmann, pp 974-985

58. Walsh N (2003) RDF Twig: accessing RDF graphs in XSLT. In: Extreme markup languages'03

59. Zhou M, Wu Y (2010) XML-based RDF data management for efficient query processing. In: Dong XL, Naumann F (eds) Proceedings of the 13th international workshop on the web and databases 2010, WebDB 2010, Indianapolis, Indiana, USA, June 6, 2010 